\title{
Unveiling unexpected complexity and multipotentiality of early heart fields
}

4 Qingquan Zhang ${ }^{1 *}$, Daniel Carlin ${ }^{1 *}$, Fugui Zhu' ${ }^{1}$, Paola Cattaneo ${ }^{1}$, Trey Ideker ${ }^{2}$, , Sylvia M.

5 Evans $^{1,4 \S}$, Joshua Bloomekatz ${ }^{1,5 \S}$ and Neil C. Chi ${ }^{1,3 \S}$

6

7 INSTITUTION

$8{ }^{1}$ Department of Medicine, Division of Cardiology, University of California San Diego, La

9 Jolla, CA 92093, USA.

$10 \quad{ }^{2}$ Department of Medicine, Division of Genetics; Department of Computer Science and

11 Engineering; Department of Bioengineering, University of California San Diego, La Jolla,

12 CA 92093, USA.

$13{ }^{3}$ Institute of Genomic Medicine, University of California, San Diego, La Jolla, CA 92093,

14 USA.

$15{ }^{4}$ Department of Pharmacology, Skaggs School of Pharmacy and Pharmaceutical Sciences,

16 University of California at San Diego, La Jolla, CA 92093, USA.

$17{ }^{5}$ Current address: Department of Biology, The University of Mississippi, University, MS

18 38677, USA.

19

20

$21 *$ contributed equally

$22 \S$ corresponding authors

23

24 Correspondence: Evans, Sylvia (syevans@ucsd.edu), Joshua Bloomekatz

25 (josh@olemiss.edu), Neil Chi (nchi@health.ucsd.edu) 


\section{Abstract}

29 Complex organs are composed of a multitude of specialized cell types which assemble to

30 form functional biological structures. How these cell types are created and organized

31 remains to be elucidated for many organs including the heart, the first organ to form during

32 embryogenesis. Here, we show the ontogeny of mammalian mesoderm at high-resolution

33 single cell and genetic lineage/clonal analyses, which revealed an unexpected complexity of

34 the contribution and multi-potentiality of mesodermal progenitors to cardiac lineages creating

35 distinct cell types forming specific regions of the heart. Single-cell transcriptomics of

36 Mespl lineage-traced cells during embryogenesis and corresponding trajectory analyses

37 uncovered unanticipated developmental relationships between these progenitors and lineages

38 including two mesodermal progenitor sources contributing to the first heart field (FHF), an

39 intraembryonic and a previously uncharacterized extraembryonic-related source, that produce

40 distinct cardiac lineages creating the left ventricle. Lineage-tracing studies revealed that

41 these extraembryonic-related FHF progenitors reside at the extraembryonic-intraembryonic

42 interface in gastrulating embryos and generate cardiac cell types that form the epicardium and

43 the dorsolateral regions of the left ventricle and atrioventricular canal myocardium. Clonal

44 analyses further showed that these progenitors are multi-potent, creating not only

45 cardiomyocytes and epicardial cell types but also extraembryonic mesoderm. Overall, these

46 results reveal unsuspected multiregional origins of the heart fields, and provide new insights

47 into the relationship between intraembryonic cardiac lineages and extraembryonic tissues and

48 the associations between congenital heart disease and placental insufficiency anomalies. 
Introduction

50 Embryonic development is a process by which a single cell with potential to give rise to all

51 cells within the embryo progressively creates groups of cells with more restricted potential.

52 A developmental field is a collection of cells with a shared potential to produce a restricted

53 subset of embryonic structures. By their nature, developmental fields are transient and

54 present only at specific developmental stages. Studies of heart development over the last

55 few decades have defined a first heart field (FHF) and a second heart field (SHF), according

56 to their potential to give rise to specific myocardial lineages within the developing heart ${ }^{1}$.

57 The FHF and SHF were inferred by retrospective clonal analyses in the mouse embryo,

58 which revealed two clonally distinct differentiated myocardial lineages, the first and second

59 heart lineages, respectively ${ }^{2-4}$. At E8.5, clones of the first heart lineage were observed to be

60 excluded from the outflow tract, populate the entire left ventricle (LV) and left

61 atrioventricular canal (AVC), and contribute some cells to both atria and right ventricle (RV),

62 whereas clones of the second heart lineage were found to be excluded from the $\mathrm{LV}^{2}$. Of the

63 heart fields predicted by this model, the SHF has been visualized and defined, as a population

64 of cells medial to the differentiating myocardial cells of the cardiac crescent that expresses

65 the transcription factor Isl1 around E7.75 . SHF cells expressing Isl1 will also give rise to

66 multiple other cell types that contribute to the heart, pharyngeal arches and head/neck

67 including endothelial, endocardial and smooth/skeletal muscle cells ${ }^{5-11}$. At E7.75, the first

68 differentiating cells in the cardiac crescent are marked by the ion channel Hcn4. As Hcn4-

69 CreERT2 labeled cells in the crescent mainly contribute to cardiomyocyte lineages in the LV

70 and parts of the atria, they are thought to represent more differentiated precursors of first

71 heart lineage cardiomyocytes, and for that reason have been considered as representatives of

72 the FHF at crescent stages ${ }^{12,13}$. However, the origins and attributes of FHF progenitors prior

73 to cardiac crescent stages remain unknown. 
In addition to myocardial and endocardial lineages, the fully formed heart includes

75 fibroblasts and vascular support cells that derive from the epicardium. The proepicardium, a

76 transient cluster of cells that forms at the base of the looping heart from the septum

77 transversum (ST) during early heart development, produces cells which cover the heart

78 surface as an epithelium to form the epicardium ${ }^{14}$. Subsets of cells from the epicardium will

79 migrate into the myocardium to give rise to cardiac fibroblasts and vascular support cells of

80 the coronary vasculature, which are essential for formation and function of the heart ${ }^{15-17}$.

81 Yet, the developmental origin of the proepicardium, and its relationship to previously

82 described heart fields remains to be defined ${ }^{18}$.

83 To address the developmental origins, definition and contribution of specific cell

84 lineages creating the heart, we performed single cell transcriptomic analyses on Mespl-cre;

85 Rosa26-tdTomato (Rosa26-tdT) mouse embryos across key developmental stages of cardiac

86 development. Computational trajectory analyses of these data notably predicted a potential

87 group of progenitors specifically expressing Hand1 that may give rise to a subset of first

88 heart lineage cardiomyocytes. Notably, in situ and lineage tracing analyses utilizing Hand1-

89 CreERT2 revealed a Hand1-expressing population at the extraembryonic/intraembryonic

90 boundary of the gastrulating embryo that contributes to first heart lineage cardiomyocytes

91 residing largely within dorsolateral regions of the LV and AVC. Intriguingly, the Hand1-

92 CreERT2 lineage created only a subset of (rather than all) LV cardiomyocytes. As the

93 second heart lineage does not populate the $\mathrm{LV}^{2}$, this finding implies a previously unexpected

94 complexity of the FHF in which the FHF is not a single developmental heart field, but rather

95 composed of at least two distinct developmental heart fields, one of which, identified here, is

96 marked by Hand1. Earlier studies have presumed that the FHF, in contrast to the SHF, has a

97 tightly restricted developmental potential, only giving rise to myocardial cells within specific

98 segments of the heart. Utilizing Hand1-CreERT2 and Rosa26-Confetti clonal analyses, we 
surprisingly found that the Hand1-CreERT2-marked FHF is composed of multipotent cells extraembryonic mesoderm.

103 Overall, our results reveal a closer lineage relationship than previously suspected between cardiac tissues and extraembryonic mesoderm. Our observation that the Hand1 expressing segment of the FHF gives rise to epicardial cells also provides insight into the developmental origins of the epicardium, thus uncovering a new early clonal relationship

107 between cardiac muscle cells of the first heart lineage and cells of the epicardium.

\section{Results}

110 scRNA-seq analysis of Mesp1 lineage-traced cells reveals developmental cell types

111 participating in mesoderm-related organogenesis.

112 As Mespl is known to mark early mesoderm, we employed a mouse Mespl-Cre ${ }^{19}$; Rosa26-

113 tdTomato $(R 26 R \text { - } t d T)^{20}$ genetic fate mapping system to permanently label and track all cell

114 lineages contributing to the development of mesoderm-derived organs including the heart

$115^{19,21-24}$ (Fig. 1a). To discover the broad spectrum of developmental cell types participating in

116 this process, we interrogated the transcriptomes of individual Mesp1-Cre; Rosa26-tdT

117 genetically-labeled cells utilizing single-cell RNA-sequencing (scRNA-seq) (Fig. 1a).

118 Because of our focus on early mesoderm-related organogenesis, we specifically examined

119 isolated Mesp1-Cre; Rosa26-tdT single cells at E7.25 (no bud stage), E7.5 (early bud stage),

120 E7.75 (late head fold stage) and E8.25 (somite stage) (Fig. 1a, Extended Data Fig. 1). Each

121 sample was processed and analyzed through our standard pipeline and confirmed for replicate

122 reproducibility (Extended Data Fig. 1a-c). t-distributed stochastic neighbor embedding

123 (tSNE) visualization and unsupervised k-means clustering ${ }^{25}$ of these combined single cell

124 data revealed a broad array of cell types, which were identified based on gene expression, 
during mesoderm development (Fig. 1b-f, Extended Data Fig. 1 and 2, Supplementary Table.

126 1). As mesodermal progenitors differentiated into organ-specific cell types during mouse

127 embryogenesis, we observed that the number of identified cell-types increased with

128 developmental age. For example, nascent, early-extraembryonic and hemogenic mesoderm

129 (NM, EEM, Hem) cell types were detected at E7.25 as previously described ${ }^{26,27}$; however,

130 many more intermediate and differentiated organ-specific cell types were identified by E7.75

131 and E8.25, including two cardiomyocyte clusters - developing cardiomyocytes (DC) and

132 cardiomyocytes (CM) (Fig. 1b). These cell clusters appear to represent early (developing)

133 and more established cardiomyocytes, respectively, based on their differential expression of

134 sarcomeric (Tnnt2, Ttn and Myl3) and cardiac progenitor genes (Tbx5, Sfrp1/5 and Meis1)

135 (Fig. 1f).

Trajectory analysis elucidates developmental pathways during mesoderm organogenesis.

139 To illuminate the developmental origins and cell fate decisions of organ-specific cell types

140 arising from mesodermal progenitor cells including cardiac cell types, we organized cells

141 from our single cell data along developmental trajectories using the lineage inference analysis, $\mathrm{URD}^{28}$, which is based on a random walk of the nearest neighbor graph of gene expression. These reconstructed developmental trajectories, as displayed in the tree structure

144 that URD produces, not only ordered cells by a pseudotime which correlate with the

145 developmental age of analyzed cells but also revealed both new and known developmental

146 cell fate decisions (Fig. 2a, Extended Data Fig. 3a). In particular, we observed

147 developmental trajectories that identified previously described mechanisms of development

148 for some discovered cell types including the early differentiation and bifurcation of

149 endothelial and blood cells ${ }^{29}$, the differentiation of somitic mesoderm from a pre-somitic state

150 originating in the caudal mesoderm ${ }^{30,31}$ and a common progenitor that gives rise to cranial 
151 pharyngeal, lateral plate mesoderm and cardiomyocytes ${ }^{1,9,11,32}$ (Fig. 2a). On the other hand,

152 examination of the cardiomyocyte developmental trajectory uncovered two potential

153 developmental sources that may contribute to developing cardiomyocytes: a known

154 intraembryonic progenitor from the lateral plate mesoderm (LPM) and a previously

155 undescribed cardiac progenitor from the late extraembryonic mesoderm (LEM) (Fig. 2a,

156 box). Viewing these developmental trajectories as a three-dimensional force-directed URD

157 representation revealed how these two progenitor sources originate and then converge to

158 independently contribute to developing cardiomyocytes (Fig. 2b, Extended Data Fig. 3b).

159 Consistent with these findings, we further discovered from a URD lineage inference analysis

160 of previously published mouse embryonic scRNA-seq data ${ }^{27}$ that analogous LPM and LEM

161 cells could be identified forming similar developmental trajectories contributing to

162 developing cardiomyocytes (Extended Data Fig. 3c-e). Thus, our bioinformatic analyses

163 support that cells with an extraembryonic signature (LEM) may contribute to the heart in a

164 trajectory that is separate from that of the embryonic (LPM) lineage.

166 Multiple developmental pathways create distinct cardiomyocyte populations.

167 Previous studies have reported the existence of distinct populations of cardiomyocytes during 168 heart development which arise from distinct heart fields ${ }^{2-4}$. Thus, we investigated whether 169 these specific cardiomyocyte populations could be detected as subclusters within our initially

170 identified developing cardiomyocyte (DC)/cardiomyocyte (CM) clusters (Fig. 1d), and

171 furthermore how LPM and LEM cells in our cardiomyocyte trajectories may specifically

172 contribute to these subclusters (Fig. 2b-magnification). As a result, subclustering analysis

173 of cells specifically comprising the initial developing cardiomyocyte/cardiomyocyte branches

174 (Fig. 2a, b-boxed area, magnification: LEM, LPM, DC and CM) uncovered seven distinct

175 sub-populations (Fig. 2c, d, Extended Data Fig. 4a, Supplementary Table 2). Three of these 
sub-clusters exhibited increased expression of cardiomyocyte sarcomeric genes such as Ttn, correlated with the CM cluster (Fig. 2c - boxed area), whereas the other four sub-clusters displayed relatively low expression of these sarcomeric genes but high expression of cardiac progenitor (CP) markers such as Isl1, Sfrp5, Tbx5 (Fig. 2c, d, Cardiac Progenitor/CP4-7, Extended Data Fig. 4a, c), and associated closely with the DC cluster and specific portions of LPM and LEM clusters (Fig. 2c - unboxed area). Differential gene marker analyses of the enrichment of Irx4/Tbx5, Tdgf1/Isl1 and Mab21l2/Tbx5, respectively, and that CM1 cells exhibited increased expression of mature cardiomyocyte gene markers including Actc1, Actn2, Myh6, Myh7 and Myll (Extended Data Fig. 4a, d). Thus, these findings indicate that CM1 and CM2 subclusters may represent cardiomyocytes arising from the FHF and SHF ${ }^{3-}$ 5,7,12,13,33-36, whereas the developmental source of the CM3 subcluster remains to be identified. Additional gene marker analyses of CP subclusters support that cell types from some of these subclusters may represent cardiac progenitors for not only cardiomyocytes but also potentially other differentiated cardiac cell types (Fig. 2d, Extended Data Fig. 4a, c). For instance, CP6 and CP7 expressed genes that overlapped with those in not only CM3 cells but also proepicardial cells (Upk3b, Ccbe1, Sfrp5, Mab21l2, Tbx1837-43) (Extended Data Fig. 4a), suggesting that $\mathrm{CP} 6 / \mathrm{CP} 7$ subclusters may contain progenitors for both $\mathrm{CM} 3$ and proepicardial cells.

To confirm the identity of potentially known subcluster cell types, annotate those that remain to be elucidated and further investigate their relationship during embryogenesis, we spatially mapped these cell types in E8.25 embryos when these cell types are present using RNAscope in situ hybridization (ISH) analysis of markers that are individually or combinatorially specific to these subclusters (Fig. 2e-k). Results from these studies revealed 
that Irx4, Tdgf1 and Mab21l2, markers of CM1, CM2 and CM3 subclusters, respectively, were expressed in three distinct regions of the heart tube as labeled by Myl7 and Nkx2-5: the middle segment (Primitive left ventricle/LV), arterial pole (Primitive outflow tract/OFT and right ventricle/RV), and venous pole of the heart tube, respectively (Fig. 2e, f), and thus indicate that $\mathrm{CM} 1$ and $\mathrm{CM} 2$ cells correspond to cardiomyocytes derived from the FHF and SHF, respectively ${ }^{3-5,7,12,13,33-36}$, whereas the source of progenitors giving rise to CM3 cardiomyocytes remains to be determined. Using a combination of genes that are differentially expressed in the CP subclusters, we further investigated the location of CP subcluster cell types during embryogenesis (Fig. 2d, g-k, Extended Data Fig. 4a, c). We

210 discovered that the combined CP4 markers $S f r p 5$ and $N r 2 f 2$ were specifically expressed in 211 regions posterior to the venous pole and contiguous with CM1 (Fig. 2g). The CP5 marker

212 Isl1 was enriched in regions anterior and dorsal to the arterial pole and contiguous with CM2

213 (Fig. 2h). The combined CP6 markers Smoc2 and Mab21l2 were expressed at the interface

214 between the forming heart and extraembryonic tissues, near the ventral venous pole and 215 contiguous with CM3 (Fig. 2i), and the combined CP7 markers Sfrp5 and Mab21l2 were 216 located in a region connected to the ventral side of the venous pole and contiguous with CP6

217 (Fig. 2j). The adjacent locations of CP6 and CP7 and a large number of overlapping genes

218 between them (Cpa2, Mab21l2, Bmp4, Hand1) (Fig. 2d, i, j, Extended Data Fig. 4a, c),

219 suggest that CP6 and CP7 may be developmentally related.

Based on these subcluster analysis findings, we further investigated the developmental relationship of the CM1-3 subpopulations and specifically how LPM and LEM progenitors may contribute to them. To this end, we reconstructed our developmental trajectories (Fig.

223 3a, b, Extended Data Fig. 5) using the three CM subcluster populations CM1-3 (Fig. 2c -

224 instead of the CM cluster from the initial tSNE cluster, Fig. 1d) as end points for our URD

225 trajectory analysis ${ }^{28}$. As a result, the modified URD developmental trajectory tree created 
three new cardiomyocyte trajectory branches for each CM subcluster (Fig. 3a, b, box). The

227 CM1 and CM2 trajectory branches, whose cells expressed genes associated with FHF (Tbx5)

229 intraembryonic cellular origin associated with LPM and NM cells, whereas the CM3 lineage

230 branch was distinct from the CM1 and CM2 branches and appeared to share an origin with

231 early and late extraembryonic mesoderm (EEM and LEM) cells (Fig. 3a, b, Extended Data

232 Fig. 5). Furthermore, the CM2 and cranial-pharyngeal ( $\mathrm{CrPh})$ branches expressed the SHF

233 marker, Isll, and appeared along a developmentally related trajectory consistent with

234 previous studies of SHF development ${ }^{5,6,8,9,11}$ (Fig. 3a-c).

Interrogating transcriptional profiles of CM1-3 lineage branches uncovers distinct cell

fate programs for each cardiomyocyte population.

238 To identify gene programs that regulate the cell fate decisions creating these distinct

239 cardiomyocyte lineages, we further interrogated the transcriptional profiles of cells along

240 each of the cardiomyocyte developmental trajectories within the URD branching tree. To

241 this end, we created a Random Forest model to classify and assign an importance score to

242 transcription factors that may participate in directing cells to a specific daughter branch at

243 each branch point examined in the URD tree ${ }^{44}$. These transcription factors were then ranked

244 based on their importance score, and the top ten transcription factors that were predicted most

245 likely to direct these branch point decisions were selected for each daughter branch

246 (Extended data Fig. 6a-c). Expanding our analysis beyond transcription factors, we further

247 identified the top twenty genes that were differentially expressed between daughter cells

248 immediately after each branch point (Extended data Fig. 6d-g, Supplementary Table 3).

249 These analyses revealed that Handl appeared important for the initial branch point decision

250 between embryonic NM and EEM (branch point 1) (Fig. 3c, Extended Data Fig. 6a-d), which

251 coincides with the role of Hand 1 in extraembryonic mesoderm development ${ }^{45,46}$. Supporting 
previous cardiac developmental studies $3,5,47-49$, we observed that the transcription factors related CM1, SHF-related CM2 and SHF-related $\mathrm{CrPh}$ cell-types at branch point 3 after their specification from intraembryonic NM/LPM cells (Figure 3c, Extended Data Fig. 6a-c, f). In the extraembryonic branch, $C d x 2 / C d x 4$ and $T s c 22 d 1$ were reciprocally expressed at branch point 2 where allantois (A) and LEM cells arise from EEM cells (Extended Data Fig. 6a, c, implicated in cardiac development ${ }^{50-54}$, and Hoxb6 and Hand1, which were expressed in the

260 LEM cells, were predicted to play key roles in regulating cell fate decisions at branch point 4 261 (Extended Data Fig. 6a-c, g).

To further illuminate the dynamics of cell fate choices and corresponding differentiation states among these cardiomyocyte lineages, we examined genes differentially expressed in each cardiomyocyte lineage trajectory along a pseudotime from least to most differentiated conditions (Extended Data Fig. 7). These pseudotime analyses revealed at least three major differentiation states for each CM trajectory: an early, intermediate and late state (Extended Data Fig. 7g-i). Consistent with our branch point analyses (Extended Data Fig. 6), genes for the CM1 and CM2 early states were similar to each other but notably distinct from those for the CM3 early state; however, genes across these pseudotime analyses converged as each intermediate state cardiac progenitor differentiated into its corresponding late state cardiomyocyte population (Extended Data Fig. 7). In particular, Mespl was expressed in the

272 CM1 and CM2 early states but $T b x 5$ and Isll were reciprocally activated in these lineages at intermediate states (Fig. 3c, Extended Data Fig. 7a, b, d, e, g, h), suggesting that CM1 and

274 CM2 may derive from a common developmental trajectory but Tbx5 and Isll may direct their specification in more distinct cardiomyocyte populations. On the other hand, Handl and

276 BMP signaling related genes Bmp4 and Msx2 were primarily expressed in CM3 early states, 
and Mab21l2 and Cpa2 were activated in CM3 intermediate states (Fig. 3c, Extended Data

278 Fig. 7c, f, i). Finally, Mef2c and other sarcomeric genes (Tnnt2) were commonly expressed

279 at the late state of CM1-3 lineages; however, some genes appeared to be specific for each CM

280 population at this state including Irx4 (CM1) and Tdgfl (CM2) (Extended Data Fig. 7d-i).

281 Confirming these analyses, RNAscope ISH revealed that Hand1 was expressed at the

282 boundary of embryonic and extraembryonic tissues, whereas Mespl was expressed in the

283 proximal portion of the intraembryonic migrating mesoderm at E7.25 and E7.5 (Fig. 3d, e).

284 Furthermore, Hand1, Tbx5 and Isl1 marked different locations in the crescent region at E7.75

285 where Handl labeled a region anterior and lateral to the cardiac crescent, which was marked

286 by $T b x 5^{3,34}$, while Isl 1 labeled cells posterior and medial to the cardiac crescent as previously

287 reported $^{5}$ (Fig. 3f, g, Extended Data Fig. 8). Altogether, these bioinformatic and spatial gene

288 expression analyses reveal a potentially unexplored developmental source of cardiomyocytes

289 along the proximal extraembryonic-embryonic boundary that may be distinct from the

290 previously described FHF and SHF progenitors.

Hand1 lineage tracing reveals an unexpected heart field that contributes to specific

subsets of the first heart lineage and serosal mesothelial lineages.

To examine and developmentally define this predicted extraembryonic-related developmental

heart field, we employed an inducible Cre-recombinase genetic fate mapping strategy to

lineage trace cells from this potential heart field during embryogenesis. Based on our interrogation of transcriptional profiles of cells along the CM URD trajectory branches, we

298 discovered that Hand 1 was expressed in early extraembryonic-related CM3 progenitors but not $\mathrm{CM} 1$ and $\mathrm{CM} 2$ progenitors, thus identifying Hand1 as a potential candidate gene to

300 genetically label progenitors from the CM3 heart field (Fig. 3c). To further explore this

301 possibility, we performed additional RNAscope ISH analyses to examine the expression of

302 Hand 1 in the developing embryo and more specifically in these distinct cardiomyocyte 
progenitors. These studies revealed that Hand1+ mesoderm cells co-expressed Mespl at the extraembryonic/embryonic boundary between E6.25 and E6.75 (Fig. 4a, Extended Data Fig.

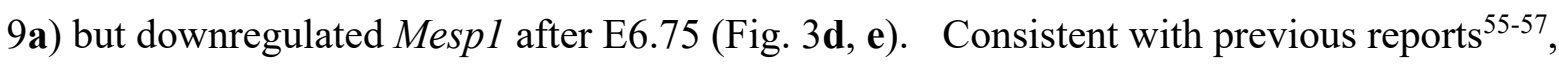
Hand1 was expressed in the extraembryonic mesoderm at E7.75, E8.25 and pericardium at

307 E8.25 but not in Hcn4+ or Myl7+ cardiomyocytes at any of these stages (Fig. 4b, c, Extended Data Fig. 9b-e). However, at E8.5 and E9.0, Hand1 was expressed in a portion of time window (E6.25 - E8.25) in which the contributions of early gastrulating Hand1 + CM3 progenitors to the heart can be investigated by Hand1-CreERT2 lineage tracing.

314 cassette into the second exon of the Handl gene (Fig. 4d, Extended Data Fig. 10a, b).

315 RNAscope ISH studies confirmed that expression of CreERT2 precisely recapitulated that of

316 Hand1 (Fig. 4e, Extended Data Fig. 10c, d). As a result, Hand1-CreERT2 mice were bred

317 with the Rosa26-tdT reporter mice to perform lineage tracing studies (Fig. 4d). Confirming the fidelity of the CreERT2 activity, Cre leakage was not observed in Hand1-CreERT2;

319 Rosa26-tdT embryos without tamoxifen induction (Extended Data Fig. 10e). Because

320 previous studies including our own (Extended Data Fig. 11) have shown that the half-life of 321 tamoxifen in mice is $\sim 12$ hours and persists over a $\sim 24-36$ hour time period ${ }^{58,59}$, we studied

322 Hand1-CreERT2; Rosa26-tdT embryos from pregnant mice given tamoxifen at E5.75 (Fig.

$3234 \mathbf{f})$ to avoid the possibility that a small amount of residual tamoxifen would activate CreERT2

324 in differentiated cardiomyocytes expressing Hand1 at E8.5. Consistent with our CM

325 trajectory branches (Fig. 3c), examination of these genetically-labeled embryos at E7.75,

326 E8.25, E8.5, E9.5 and E12.5 revealed that Hand1 lineage-traced cells contributed to not only 327 extraembryonic tissue but also the heart (Fig. 4g-n, Extended Data Fig. 12). Within 
328

329

330

331

332

extraembryonic tissues, which were tdT-labeled throughout the yolk sac across all examined stages (Fig. 4g-l, Extended Data Fig. 12b), Handl lineage-traced tdT + cells produced Pecam + endothelial cells, $\alpha-S M A+$ smooth muscle cells and Pdgfr $\beta+$ mesothelial cells (Extended Data Fig. 12e, f). On the other hand, Hand1 lineage-traced tdT + cells contributed to the developing embryo in a more spatial and temporal restricted manner (Fig. 4g-n). Specifically, Hand1 lineage-traced tdT + cells supplied Hcn4+ cardiomyocytes in the cardiac crescent at E7.75 and then cardiomyocytes $(M y l 7+)$ on the ventral side of the venous pole and medial regions of the heart tube at E8.25 (Fig. 4g-j). At later stages (E8.5 - E12.5), tdT+ cardiomyocytes were increasingly restricted spatially to the primitive AVC region and LV at E8.5 and then further to the AVC/sinus venosus (SV), dorsolateral LV and atrial regions of the heart at E12.5 (Fig. 4k-n, Extended Data Fig. 12b, d). Furthermore, tdT + cells appeared in non-myocardial heart tissue including the pericardium, proepicardium/ST, epicardium, and occasionally endocardium from E8.25 - E12.5 (Fig. 4i-n, Extended Data Fig. 12a-d).

Supporting these lineage studies, the CM3 URD tree branch, which included the CP6 and CP7 subclusters, comprised cells that express AVC markers (Msx1/2, Twist1, Tbx $\left.2^{42,60-62}\right)$, as well as proepicardial/pericardial markers (Upk3b, Ccbe1, Sfrp5, Mab2112, Tbx18 ${ }^{37-43}$ ) (Extended Data Fig. 4a). Finally, to confirm these findings and investigate additional early gastrulating Hand1 $+\mathrm{CM} 3$ progenitors that may not have been labeled at E5.75, we examined Hand1-CreERT2; Rosa26-tdT embryos from pregnant mice given tamoxifen at E6.25 (Extended Data Fig. 13). In addition to displaying similar results to those observed in E5.75 tamoxifen-induced Hand1-CreERT2; Rosa26-tdT embryos (Extended Data Fig. 13a-f compared to Fig. 4g-n), Hand1 lineage-traced tdT+ cells genetically-labeled at E6.25 also contributed cardiomyocytes as well as all epicardial-derived cell-types including fibroblasts and vascular support cells to E17.5 embryos (Extended Data Fig. 13g). Altogether, these data suggest that at early gastrulation stages, Hand1 marks a progenitor population that gives 
rise not only to cardiomyocytes within the AVC and LV prior to the time when Handl is actively expressed in differentiated CMs, but also to pericardial, epicardial and extraembryonic derived mesoderm cell types. As these Hand $1+$ cardiomyocyte progenitors specifically contribute to cardiomyocytes within the developing atrioventricular canal and dorsolateral regions of the LV, they likely represent a distinct subset of the reported first heart lineage cardiomyocytes ${ }^{2}$, suggesting that the FHF is not a single heart field, but is rather composed of at least two distinct heart fields, one of which, identified here, is marked by Hand1.

\section{Genetic clonal analysis reveals the multipotentiality of $\mathrm{Hand1}+$ cardiac precursor cells.}

363 Our lineage tracing results reveal that Hand1+ progenitors in the early gastrulating embryo

364 give rise to multiple distinct cell types in extraembryonic tissue, pericardium, endocardium, epicardium as well as the dorsal LV and AVC myocardium in the developing heart (Fig. 4, Extended Data Fig. 12, 13). These findings may reflect the presence of distinct Hand1+ precursor cells that produce individual cell types, or multipotential Hand1+ precursor cells which can differentiate into different combinations of cell types. To examine the lineage potential of single Hand 1 -expressing cells during early gastrulation, we crossed HandlCreERT2 mice with the Rosa26-Confetti multicolor reporter mice ${ }^{63}$ to genetically fate map early Hand $1+$ individual clones expressing a specific fluorescent protein following low dose tamoxifen treatment at E6.75 or E7.25 (Fig. 5a). To this end, we discovered that $0.005 \mathrm{mg} / \mathrm{g}$ of tamoxifen was the minimum effective dose at E6.75 or E7.25 that reliably leads to clonal events in examined embryos (Extended Data Fig. 14a, b). This dose resulted in recombination in only $27 \%$ of embryos $(n=175 / 640)$, which was less than the expected Hand1-CreERT2; Rosa26-confetti genotype positivity rate (50\%) (Extended Data Fig. 14b).

377 Among these embryos, bi-color embryos occurred in the highest proportion followed by uni378 color and tri-color embryos (Fig. 5b), and the observed frequency of each Brainbow color 
(YFP, RFP, CFP and nGFP) was consistent with those from previous reports ${ }^{4}$ (Extended Data

380 Fig. 14c). Further examining all labeled embryos at E9.5 revealed that $\sim 73 \%(242 / 330)$ of

381 the clones were present in only the extraembryonic tissue; $26 \%(86 / 330)$ were located in

382 both extraembryonic and cardiac tissue; and two clones $(\sim 1 \%)$ contributed to only cardiac

383 tissue (Extended Data Fig. 14d). The distribution of these clones combined together were consistent with the distribution of genetically-labeled Hand1-CreERT2; Rosa26-tdT cells at a similar stage (Fig. 4k, l, compared to Fig. 5c-e). Supporting the multipotentiality of early Hand $1+$ progenitors during early gastrulation, uni-color embryos, which were most likely due to a single recombination event ${ }^{4}$, exhibited clones that fluorescently-labeled both extraembryonic and cardiac tissues including the proepicardium/ST, pericardium, LV and AVC at E9.5 (Fig. 5c-e). Additional immunofluorescence studies revealed that these Hand $1+$ clones specifically contributed to not only $\alpha$-Actinin + cardiomyocytes in the AVC and LV but also Wt1+ proepicardial and pericardial cells in the developing embryo (Fig. 5c, Extended Data Fig. 14e-h). However, consistent with the relatively few endocardial cells genetically labeled in Hand1-CreERT2; Rosa26-tdT embryos (Fig. 4k', Extended Data Fig. 12d'), Hand $1+$ clones were not observed in the endocardium. Finally, Hand1+ clones supplied $\alpha$-SMA + smooth muscle cells and Pdgfr $\beta+$ mesothelial cells to the extraembryonic mesoderm (Extended Data Fig. 14i, j).

To further substantiate that clones in both the yolk sac and heart derive from a single recombination event, we examined additional embryos containing bi- and tri-color clones.

399 To ensure that these clones resulted from single recombination events, we employed a rigorous statistical analysis of the number cells in each clone. To this end, we counted the cells in each clone (an individual color) in either cardiac tissues or both extraembryonic and cardiac tissues, and modeled these cell counts with a mixture of two Gaussian distributions ${ }^{64}$ : one for the cell count that would be expected for a single recombination event, and the other 
404 for the cell count that would be expected for two or more recombination events (Extended

405 Data Fig.14m-p). Based on this model, we found that 53 out of 88 observed clones labeled 406 at E6.75 or E7.25 corresponded to single clonal events in both cell count analyses (Extended

407 Data Fig. 14m-p). Additional analyses of these consensus single clonal events revealed that 408 the majority of single clones in the heart contributed to two or three distinct lineages,

409 including various combinations of extraembryonic mesoderm, pericardium,

410 proepicardium/ST, and AVC or LV myocardium (Extended Data Fig. 14n, p), thus

411 supporting the multipotentiality of Handl+ progenitor cells.

412 To further investigate the clonal relationships among specific Hand1+ progenitor-

413 derived cardiac cell types and their location in later stage hearts, we examined E6.75 or E7.25

414 tamoxifen-induced Hand1-CreERT2; Rosa26-Confetti clones at E12.5 when most cardiac

415 structures and cell types have been determined. Only uni-color hearts were analyzed as

416 these hearts were most likely to be derived from a single recombination event ${ }^{4}$. Consistent

417 with the E9.5 clonal analysis (Fig. 5c), clones marking the epicardium also labeled

418 cardiomyocytes in the AVC or LV at E12.5 (Fig. 5f-i, Extended Data Fig. 14k), thus

419 supporting that multipotential Handl + cardiac progenitor cells can give rise to both

420 cardiomyocytes and non-cardiomyocytes. Altogether, these results reveal the existence of

421 multipotential Handl cardiac progenitors in the early ingressing mesoderm that can give rise

422 to extraembryonic mesoderm, mesothelial lineages (epicardium and pericardium) and LV and

423 AVC myocardium (Fig. 5j).

\section{Discussion}

426 Overall, our transcriptional and developmental interrogation of Mespl-lineages at single-cell

427 resolution has illuminated the intricacies of building complex organs/tissues derived from the

428 mesoderm. Our single-cell transcriptomic studies reveal not only well-established but also

429 previously unappreciated developmental sources for key cell lineages creating both intra- and 
extra-embryonic organs/tissues. Similar to previous studies for gut endoderm ${ }^{65}$, our

431 trajectory analysis of our developing mesoderm single cell data has uncovered a close

432 developmental relationship between intra- and extra-embryonic derived organs/tissues

433 including unexpectedly a distinct developmental lineage of the heart that is related to those

434 contributing to specific extraembryonic structures. Utilizing a combination of genetic fate-

435 mapping and clonal analyses, we not only confirm this developmental cardiac-

436 extraembryonic tissue connection but also delineate the progenitors creating these lineages

437 and their specific contributions to the developing heart and extraembryonic structures (Fig.

$4385 \mathbf{j})$.

439 Highlighting the complexity of organogenesis, we show how similar cell types, such as

440 cardiomyocytes, can derive from multiple developmental origins/progenitors that have

441 potential to contribute not only to other cell types but also to multiple organs/tissue

442 structures. In particular, further single-cell subcluster analyses of isolated cardiomyocyte

443 transcriptomic profiles identified at least three distinct myocardial heart lineages including a

444 heart lineage whose progenitor shares a gene signature with extraembryonic mesodermal

445 progenitors including Hand1. Trajectory analyses predicted that two of these heart lineages

446 derive from a common embryonic source prior to E7.25, with marker expression at E8.25

447 suggesting their correspondence to first and second heart lineages ${ }^{1}$, whereas the Hand1+

448 extraembryonic-related heart lineage originates from a distinct developmental source that

449 downregulates Mesp1 prior to E7.25 and gives rise to myocardial lineages. Further

450 expression analyses revealed that at early gastrula stages, Hand1+ progenitors reside at the

451 intra-/extra-embryonic boundary, with genetic fate mapping demonstrating that Handl+

452 progenitors specifically contribute to myocardial cells localized to the dorsal regions of the

453 LV and AVC at E12.5. As myocardial lineages contributing to the LV have previously been

454 defined as first heart lineages deriving from the $\mathrm{FHF}^{2}$, our results support that this Hand1+ 
cardiac progenitor field represents a distinct subset of the FHF, thus revealing that the FHF

457 findings are consistent with a mathematically-inferred myocardial lineage model from

458 previous retrospective clonal analyses ${ }^{2-4}$, thus further supporting our findings.

459 One limitation for understanding the full lineage potential of the FHF or SHF from

460 retrospective clonal studies ${ }^{2}$, is that, because of experimental design, only myocardial clones

461 can be studied. However, when Isll was identified as a marker of the SHF, studies with

462 Isl1-Cre or inducible Isl1-CreERT2 revealed that the SHF produces both myocardial lineages

463 as well as multiple other cardiac lineages ${ }^{5,8,66,67}$. Here, utilizing Hand1-CreERT2 in concert

464 with a confetti clonal indicator ${ }^{63}$, we uncovered an unsuspected multipotentiality of the

465 Handl FHF in which cells within the Handl FHF can give rise not only to a specific subset

466 of myocardial lineages within the first heart lineage, but also to extraembryonic mesoderm,

467 septum transversum/epicardial, and pericardial cells. Thus, our results reveal that

468 myocardial cells of the AVC and LV (particularly dorsal regions) and extraembryonic

469 mesodermal and serosal mesothelial cells have a closer lineage relationship than previously

470 expected, while also addressing the elusive embryonic origins of the

471 proepicardium/epicardium, which contributes essential vascular support cells and cardiac

472 fibroblasts to the heart.

473 The existence of a progenitor population that gives rise to cells both within

474 extraembryonic and intraembryonic tissues provides a further example of blurred boundaries

475 between extraembryonic and intraembryonic tissues, as seen by migration of extraembryonic

476 hematopoietic progenitors to intraembryonic sites ${ }^{68}$, and intercalation of extraembryonic and

477 intraembryonic endoderm during gut formation ${ }^{69}$. Additionally, these findings may also

478 account for previous observations that, under certain in vitro conditions, epicardial

479 progenitors can adopt cardiomyocyte cell fates ${ }^{70}$, and that loss of an 
endothelial/hematopoietic transcription factor, Scl, can result in transdifferentiation of yolk

Hand1 + FHF-derived cardiac lineages, coupled to the high plasticity of mesothelial cells ${ }^{72-74}$, suggests the possibility of transforming extraembryonic and serosal mesothelial tissues into cardiomyocytes to treat heart failure in the future.

As Hand 1 marks a subset of the FHF in the early gastrula embryo, and these progenitors have multipotentiality, the specific role of Handl in early specification of FHF progenitors will be of great interest to examine in future studies. Global knockout of Handl results in embryonic lethality at approximately E8.5, and mutant embryos exhibit placental, yolk sac and heart defects ${ }^{45,46}$. As placenta and yolk sac defects can secondarily impact the heart, direct requirements for Handl in early heart progenitors remains unclear. Although some experiments, including cardiac-specific conditional knockout and tetraploid rescue studies $^{45,46}$, confirmed heart defects in Handl mutant embryos, these studies could not rule out requirements for Handl in differentiated cardiomyocytes, rather than undifferentiated progenitors. However, our findings suggest that these heart defects may be due to abnormalities in undifferentiated progenitors which can give rise to cardiomyocytes.

Because these progenitors can also contribute to extraembryonic tissue, they also raise the possibility that congenital heart diseases thought to be caused by placental anomalies ${ }^{75}$ may be due to perturbations of complex interplays between genetic pathways shared by extraembryonic and cardiac lineages.

Overall, our studies reveal that there are distinct subsets of the FHF that contribute to

502 specific corresponding subpopulations of first myocardial lineages ${ }^{2}$, and that Handl-FHF

503 progenitors are multipotential, giving rise to multiple cell lineages, including cardiovascular

504 lineages within the heart and extraembryonic cell types within the yolk sac. The 
505 cardiovascular multipotentiality of FHF progenitors, as previously seen for the SHF, may

506 further reflect the evolution of the cardiovascular system, thus highlighting the overall

507 complexity of how diverse cell types are created to build and organize functional

508 organs/tissues. 


\section{Main References}

Meilhac, S. M. \& Buckingham, M. E. The deployment of cell lineages that form the mammalian heart. Nature reviews. Cardiology 15, 705-724, doi:10.1038/s41569-0180086-9 (2018).

2 Meilhac, S. M., Esner, M., Kelly, R. G., Nicolas, J. F. \& Buckingham, M. E. The clonal origin of myocardial cells in different regions of the embryonic mouse heart. Developmental cell 6, 685-698, doi:10.1016/s1534-5807(04)00133-9 (2004).

3 Devine, W. P., Wythe, J. D., George, M., Koshiba-Takeuchi, K. \& Bruneau, B. G. Early patterning and specification of cardiac progenitors in gastrulating mesoderm. eLife $\mathbf{3}$, doi:10.7554/eLife.03848 (2014).

4 Lescroart, F. et al. Early lineage restriction in temporally distinct populations of Mesp1 progenitors during mammalian heart development. Nature cell biology 16, 829-840, doi:10.1038/ncb3024 (2014).

5 Cai, C. L. et al. Is11 identifies a cardiac progenitor population that proliferates prior to differentiation and contributes a majority of cells to the heart. Developmental cell 5, 877-889, doi:10.1016/s1534-5807(03)00363-0 (2003).

6 Kelly, R. G., Brown, N. A. \& Buckingham, M. E. The arterial pole of the mouse heart forms from Fgf10-expressing cells in pharyngeal mesoderm. Developmental cell 1, 435-440, doi:10.1016/s1534-5807(01)00040-5 (2001).

7 Prall, O. W. et al. An Nkx2-5/Bmp2/Smad1 negative feedback loop controls heart progenitor specification and proliferation. Cell 128, 947-959, doi:10.1016/j.cell.2007.01.042 (2007).

8 Bu, L. et al. Human ISL1 heart progenitors generate diverse multipotent cardiovascular cell lineages. Nature 460, 113-117, doi:10.1038/nature08191 (2009).

9 Lescroart, F. et al. Clonal analysis reveals common lineage relationships between head muscles and second heart field derivatives in the mouse embryo. Development (Cambridge, England) 137, 3269-3279, doi:10.1242/dev.050674 (2010).

10 Lescroart, F. et al. Clonal analysis reveals a common origin between nonsomite-derived neck muscles and heart myocardium. Proceedings of the National Academy of Sciences of the United States of America 112, 1446-1451, doi:10.1073/pnas.1424538112 (2015).

11 Diogo, R. et al. A new heart for a new head in vertebrate cardiopharyngeal evolution. Nature 520, 466-473, doi:10.1038/nature14435 (2015).

12 Liang, X. et al. HCN4 dynamically marks the first heart field and conduction system precursors. Circulation research 113, 399-407, doi:10.1161/circresaha.113.301588 (2013).

13 Später, D. et al. A HCN4+ cardiomyogenic progenitor derived from the first heart field and human pluripotent stem cells. Nature cell biology 15, 1098-1106, doi:10.1038/ncb2824 (2013).

14 Komiyama, M., Ito, K. \& Shimada, Y. Origin and development of the epicardium in the mouse embryo. Anatomy and embryology 176, 183-189, doi:10.1007/bf00310051 (1987).

15 Mikawa, T. \& Gourdie, R. G. Pericardial mesoderm generates a population of coronary smooth muscle cells migrating into the heart along with ingrowth of the epicardial organ. Developmental biology 174, 221-232, doi:10.1006/dbio.1996.0068 (1996).

16 Gittenberger-de Groot, A. C., Vrancken Peeters, M. P., Mentink, M. M., Gourdie, R. G. \& Poelmann, R. E. Epicardium-derived cells contribute a novel population to the myocardial wall and the atrioventricular cushions. Circulation research 82, 1043-1052, doi:10.1161/01.res.82.10.1043 (1998). 
602

603

604

605

606

607

17 Grieskamp, T., Rudat, C., Lüdtke, T. H., Norden, J. \& Kispert, A. Notch signaling regulates smooth muscle differentiation of epicardium-derived cells. Circulation research 108, 813-823, doi:10.1161/circresaha.110.228809 (2011).

18 Maya-Ramos, L., Cleland, J., Bressan, M. \& Mikawa, T. Induction of the Proepicardium. Journal of developmental biology 1, 82-91, doi:10.3390/jdb1020082 (2013).

19 Saga, Y. et al. MesP1 is expressed in the heart precursor cells and required for the formation of a single heart tube. Development (Cambridge, England) 126, 3437-3447 (1999).

20 Madisen, L. et al. A robust and high-throughput Cre reporting and characterization system for the whole mouse brain. Nature neuroscience 13, 133-140, doi:10.1038/nn.2467 (2010).

21 Oginuma, M., Hirata, T. \& Saga, Y. Identification of presomitic mesoderm (PSM)specific Mesp1 enhancer and generation of a PSM-specific Mesp1/Mesp2-null mouse using BAC-based rescue technology. Mechanisms of development 125, 432-440, doi:10.1016/j.mod.2008.01.010 (2008).

22 Yoshida, T., Vivatbutsiri, P., Morriss-Kay, G., Saga, Y. \& Iseki, S. Cell lineage in mammalian craniofacial mesenchyme. Mechanisms of development 125, 797-808, doi:10.1016/j.mod.2008.06.007 (2008).

23 Harel, I. et al. Distinct origins and genetic programs of head muscle satellite cells. Developmental cell 16, 822-832, doi:10.1016/j.devcel.2009.05.007 (2009).

24 Chan, S. S. et al. Mesp1 patterns mesoderm into cardiac, hematopoietic, or skeletal myogenic progenitors in a context-dependent manner. Cell stem cell 12, 587-601, doi:10.1016/j.stem.2013.03.004 (2013).

25 Butler, A., Hoffman, P., Smibert, P., Papalexi, E. \& Satija, R. Integrating single-cell transcriptomic data across different conditions, technologies, and species. Nature biotechnology 36, 411-420, doi:10.1038/nbt.4096 (2018).

26 Scialdone, A. et al. Resolving early mesoderm diversification through single-cell expression profiling. Nature 535, 289-293, doi:10.1038/nature18633 (2016).

27 Pijuan-Sala, B. et al. A single-cell molecular map of mouse gastrulation and early organogenesis. Nature 566, 490-495, doi:10.1038/s41586-019-0933-9 (2019).

28 Farrell, J. A. et al. Single-cell reconstruction of developmental trajectories during zebrafish embryogenesis. Science (New York, N.Y.) 360, doi:10.1126/science.aar3131 (2018).

29 Ueno, H. \& Weissman, I. L. Clonal analysis of mouse development reveals a polyclonal origin for yolk sac blood islands. Developmental cell 11, 519-533, doi:10.1016/j.devcel.2006.08.001 (2006).

30 Tam, P. P. The allocation of cells in the presomitic mesoderm during somite segmentation in the mouse embryo. Development (Cambridge, England) 103, 379-390 (1988).

31 Gouti, M. et al. A Gene Regulatory Network Balances Neural and Mesoderm Specification during Vertebrate Trunk Development. Developmental cell 41, 243261.e247, doi:10.1016/j.devcel.2017.04.002 (2017).

32 Harel, I. et al. Pharyngeal mesoderm regulatory network controls cardiac and head muscle morphogenesis. Proceedings of the National Academy of Sciences of the United States of America 109, 18839-18844, doi:10.1073/pnas.1208690109 (2012).

33 Bao, Z. Z., Bruneau, B. G., Seidman, J. G., Seidman, C. E. \& Cepko, C. L. Regulation of chamber-specific gene expression in the developing heart by Irx4. Science (New York, N.Y.) 283, 1161-1164, doi:10.1126/science.283.5405.1161 (1999).

34 Bruneau, B. G. et al. Chamber-specific cardiac expression of Tbx5 and heart defects in 
Holt-Oram syndrome. Developmental biology 211, 100-108, doi:10.1006/dbio.1999.9298 (1999).

35 van den Berg, G. et al. A caudal proliferating growth center contributes to both poles of the forming heart tube. Circulation research 104, 179-188, doi:10.1161/circresaha.108.185843 (2009).

36 Barnes, R. M. et al. MEF2C regulates outflow tract alignment and transcriptional control of Tdgf1. Development (Cambridge, England) 143, 774-779, doi:10.1242/dev.126383 (2016).

37 Rudat, C. et al. Upk3b is dispensable for development and integrity of urothelium and mesothelium. PloS one 9, e112112, doi:10.1371/journal.pone.0112112 (2014).

38 Facucho-Oliveira, J., Bento, M. \& Belo, J. A. Ccbe1 expression marks the cardiac and lymphatic progenitor lineages during early stages of mouse development. The International journal of developmental biology 55, 1007-1014, doi:10.1387/ijdb.113394jf (2011).

39 Fujii, M. et al. Sfrp5 identifies murine cardiac progenitors for all myocardial structures except for the right ventricle. Nature communications 8, 14664, doi:10.1038/ncomms14664 (2017).

40 Saito, Y., Kojima, T. \& Takahashi, N. Mab2112 is essential for embryonic heart and liver development. PloS one 7, e32991, doi:10.1371/journal.pone.0032991 (2012).

41 Kraus, F., Haenig, B. \& Kispert, A. Cloning and expression analysis of the mouse Tbox gene Tbx18. Mechanisms of development 100, 83-86, doi:10.1016/s09254773(00)00494-9 (2001).

42 de Soysa, T. Y. et al. Single-cell analysis of cardiogenesis reveals basis for organ-level developmental defects. Nature 572, 120-124, doi:10.1038/s41586-019-1414-x (2019).

43 Lupu, I. E., Redpath, A. N. \& Smart, N. Spatiotemporal Analysis Reveals Overlap of Key Proepicardial Markers in the Developing Murine Heart. Stem cell reports 14, 770787, doi:10.1016/j.stemcr.2020.04.002 (2020).

44 Di Bella, D. J. et al. Molecular Logic of Cellular Diversification in the Mammalian Cerebral Cortex. bioRxiv, 2020.2007.2002.185439, doi:10.1101/2020.07.02.185439 (2020).

45 Riley, P., Anson-Cartwright, L. \& Cross, J. C. The Hand1 bHLH transcription factor is essential for placentation and cardiac morphogenesis. Nature genetics 18, 271-275, doi:10.1038/ng0398-271 (1998).

46 Firulli, A. B., McFadden, D. G., Lin, Q., Srivastava, D. \& Olson, E. N. Heart and extraembryonic mesodermal defects in mouse embryos lacking the bHLH transcription factor Hand1. Nature genetics 18, 266-270, doi:10.1038/ng0398-266 (1998).

47 Srivastava, D., Cserjesi, P. \& Olson, E. N. A subclass of bHLH proteins required for cardiac morphogenesis. Science (New York, N.Y.) 270, 1995-1999, doi:10.1126/science.270.5244.1995 (1995).

48 Merscher, S. et al. TBX1 is responsible for cardiovascular defects in velo-cardiofacial/DiGeorge syndrome. Cell 104, 619-629, doi:10.1016/s0092-8674(01)00247-1 (2001).

49 Zhang, Z., Huynh, T. \& Baldini, A. Mesodermal expression of Tbx1 is necessary and sufficient for pharyngeal arch and cardiac outflow tract development. Development (Cambridge, England) 133, 3587-3595, doi:10.1242/dev.02539 (2006).

50 Lin, Q., Schwarz, J., Bucana, C. \& Olson, E. N. Control of mouse cardiac morphogenesis and myogenesis by transcription factor MEF2C. Science (New York, N.Y.) 276, 1404-1407, doi:10.1126/science.276.5317.1404 (1997).

51 Jongbloed, M. R. et al. Expression of Id2 in the second heart field and cardiac defects in Id2 knock-out mice. Developmental dynamics : an official publication of the 
661

662

663

664

665

666

667

668

669

670

671

672

673

674

675

676

677

678

679

680

681

682

683

684

685

686

687

688

689

690

691

692

693

694

695

696

697

698

699

700

701

702

703

704

705

706

707

American Association of Anatomists 240, 2561-2577, doi:10.1002/dvdy.22762 (2011).

52 Moskowitz, I. P. et al. A molecular pathway including Id2, Tbx5, and Nkx2-5 required for cardiac conduction system development. Cell 129, 1365-1376, doi:10.1016/j.cell.2007.04.036 (2007).

53 Fraidenraich, D. et al. Rescue of cardiac defects in id knockout embryos by injection of embryonic stem cells. Science (New York, N.Y.) 306, 247-252, doi:10.1126/science.1102612 (2004).

54 Bamforth, S. D. et al. Cited2 controls left-right patterning and heart development through a Nodal-Pitx2c pathway. Nature genetics 36, 1189-1196, doi:10.1038/ng1446 (2004).

55 Barnes, R. M. et al. Hand2 loss-of-function in Hand1-expressing cells reveals distinct roles in epicardial and coronary vessel development. Circulation research 108, 940949, doi:10.1161/circresaha.110.233171 (2011).

56 Biben, C. \& Harvey, R. P. Homeodomain factor Nkx2-5 controls left/right asymmetric expression of bHLH gene eHand during murine heart development. Genes \& development 11, 1357-1369, doi:10.1101/gad.11.11.1357 (1997).

57 Thomas, T., Yamagishi, H., Overbeek, P. A., Olson, E. N. \& Srivastava, D. The bHLH factors, dHAND and eHAND, specify pulmonary and systemic cardiac ventricles independent of left-right sidedness. Developmental biology 196, 228-236, doi:10.1006/dbio.1998.8849 (1998).

58 Robinson, S. P., Langan-Fahey, S. M., Johnson, D. A. \& Jordan, V. C. Metabolites, pharmacodynamics, and pharmacokinetics of tamoxifen in rats and mice compared to the breast cancer patient. Drug metabolism and disposition: the biological fate of chemicals 19, 36-43 (1991).

59 Wilson, C. H. et al. The kinetics of ER fusion protein activation in vivo. Oncogene 33, 4877-4880, doi:10.1038/onc.2014.78 (2014).

60 Aanhaanen, W. T. et al. The Tbx2+ primary myocardium of the atrioventricular canal forms the atrioventricular node and the base of the left ventricle. Circulation research 104, 1267-1274, doi:10.1161/circresaha.108.192450 (2009).

61 Li, G. et al. Single cell expression analysis reveals anatomical and cell cycle-dependent transcriptional shifts during heart development. Development (Cambridge, England) 146, doi:10.1242/dev.173476 (2019).

62 Harrelson, Z. et al. Tbx2 is essential for patterning the atrioventricular canal and for morphogenesis of the outflow tract during heart development. Development (Cambridge, England) 131, 5041-5052, doi:10.1242/dev.01378 (2004).

63 Snippert, H. J. et al. Intestinal crypt homeostasis results from neutral competition between symmetrically dividing Lgr5 stem cells. Cell 143, 134-144, doi:10.1016/j.cell.2010.09.016 (2010).

64 Benaglia, T., Chauveau, D., Hunter, D., R. \& Young, D., S. mixtools: An R Package for Analyzing Finite Mixture Models. Journal of Statistical Software 32, 1-29 (2009).

65 Nowotschin, S. et al. The emergent landscape of the mouse gut endoderm at single-cell resolution. Nature 569, 361-367, doi:10.1038/s41586-019-1127-1 (2019).

66 Moretti, A. et al. Multipotent embryonic isl1+ progenitor cells lead to cardiac, smooth muscle, and endothelial cell diversification. Cell 127, 1151-1165, doi:10.1016/j.cell.2006.10.029 (2006).

67 Sun, Y. et al. Islet 1 is expressed in distinct cardiovascular lineages, including pacemaker and coronary vascular cells. Developmental biology 304, 286-296, doi:10.1016/j.ydbio.2006.12.048 (2007).

68 Orkin, S. H. \& Zon, L. I. Hematopoiesis: an evolving paradigm for stem cell biology. Cell 132, 631-644, doi:10.1016/j.cell.2008.01.025 (2008). 
69 Kwon, G. S., Viotti, M. \& Hadjantonakis, A. K. The endoderm of the mouse embryo arises by dynamic widespread intercalation of embryonic and extraembryonic lineages. Developmental cell 15, 509-520, doi:10.1016/j.devcel.2008.07.017 (2008). pericardial mesoderm into the myocardial or epicardial lineage. Developmental biology 295, 507-522, doi:10.1016/j.ydbio.2006.03.033 (2006).

71 Van Handel, B. et al. Scl represses cardiomyogenesis in prospective hemogenic endothelium and endocardium. Cell 150, 590-605, doi:10.1016/j.cell.2012.06.026 (2012).

72 Zhou, B. et al. Adult mouse epicardium modulates myocardial injury by secreting paracrine factors. The Journal of clinical investigation 121, 1894-1904, doi:10.1172/jci45529 (2011).

73 Smart, N. et al. De novo cardiomyocytes from within the activated adult heart after injury. Nature 474, 640-644, doi:10.1038/nature10188 (2011). Zangi, L. et al. Insulin-Like Growth Factor 1 Receptor-Dependent Pathway Drives Epicardial Adipose Tissue Formation After Myocardial Injury. Circulation 135, 59-72, doi:10.1161/circulationaha.116.022064 (2017).

75 Matthiesen, N. B. et al. Congenital Heart Defects and Indices of Placental and Fetal Growth in a Nationwide Study of 924422 Liveborn Infants. Circulation 134, 15461556, doi:10.1161/circulationaha.116.021793 (2016). 
729 We thank Jianlin Zhang and Mi Tran for mouse care, and Evans and Chi lab members for

730 comments on the manuscript. Various experiments were conducted with the assistance,

731 expertise, and support of the following University of California San Diego (UCSD) core

732 facilities including the: Institute for Genomic Medicine Core, Mouse Transgenic Core and

733 Histology and Immunohistochemistry Core. This work was supported in part by grants from

734 the NIH to S.M.E., N.C.C.; CIRM to T. I., N.C.C.; AHA to J.B.

735

736 Author Contributions

737 Q.Z., D.C., P.C., S.M.E., J.B. and N.C.C. conceived the project and the design of the

738 experimental strategy. Q.Z., J.B. carried out experimental studies including in situ

739 hybridization study, lineage tracing and clonal analysis. D.C. performed bioinformatic

740 analysis. J.B. carried out scRNA-seq experimental studies. F.Z. generated Hand1-

741 CreERT2 knock in mice line. T. I provided bioinformatics analysis guidance. Q.Z., D.C.,

742 S.M.E., J.B. and N.C.C. prepared the manuscript.

743

744 Competing interests

745 The authors declare no competing interests.

747 Reprints and permission information is available at www.nature.com/reprints.

749 Correspondence and requests for materials should be addressed to N.C.C.

750 (nchi@health.ucsd.edu), J. B. (josh@olemiss.edu) and S.M.E. (syevans@health.ucsd.edu). 


\section{Figure 1}

a
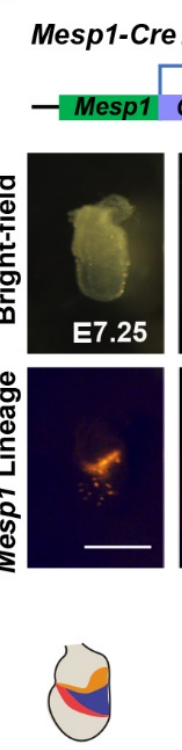

Mesp1
expressing

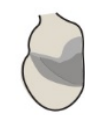

Embryos

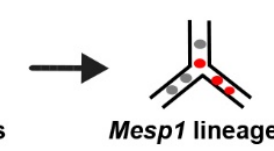

Rosa26-tdT

loxp loxp

-CAG Stop + tdTomato
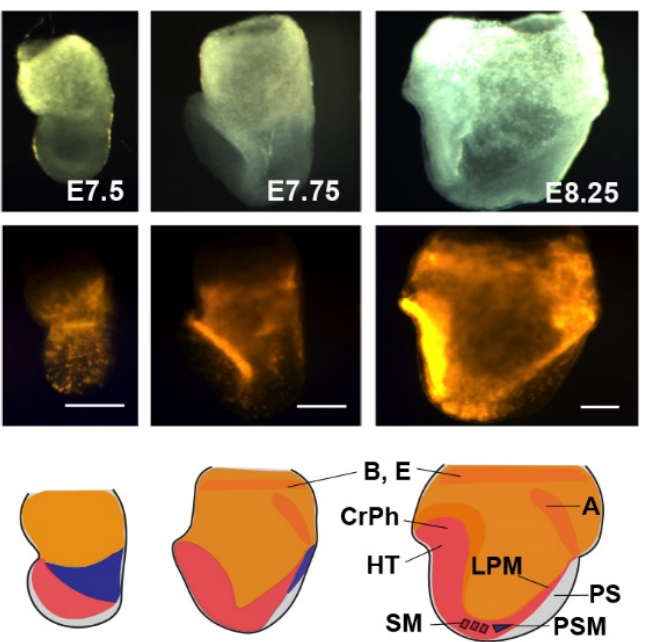

Extraembryonic

Mesp1 lineage

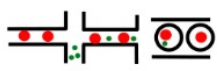

10x scRNA-seq b
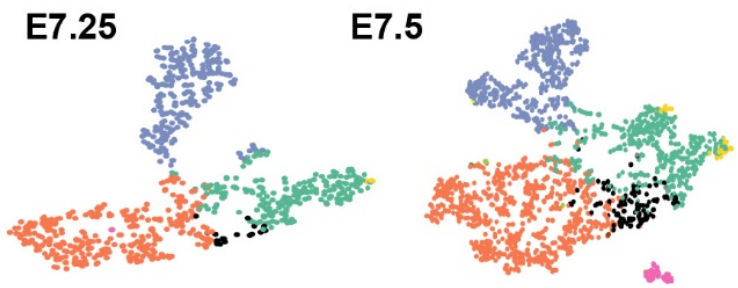

E7.75

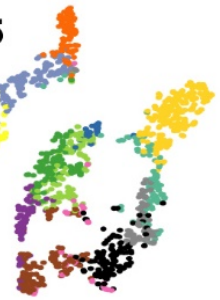

$\begin{array}{ll}1 & \text { Cardiomyocytes (CM) } \\ 2 & \text { Developing }\end{array}$ Cardiomyocytes (DC)

3 Cranial Pharyngeal Mesoderm (CrPh)

4 Somite Mesoderm (SM)

5 Endothelium (E)

6 Blood (B)

7 Pre-somitic Mesoderm (PSM)

8 Epithelium (Ep)
E8.25

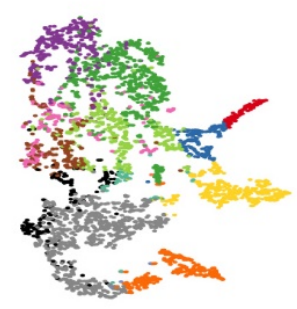

9 Allantois (A)

10 Early Extraembryonic Mesoderm (EEM)

11 Nascent Mesoderm (NM)

12 Hemogenic Mesoderm (Hem)

13 Caudal Mesoderm (CDM)

14 Lateral Plate Mesoderm (LPM)

15 Late Extraembryonic Mesoderm (LEM)
C

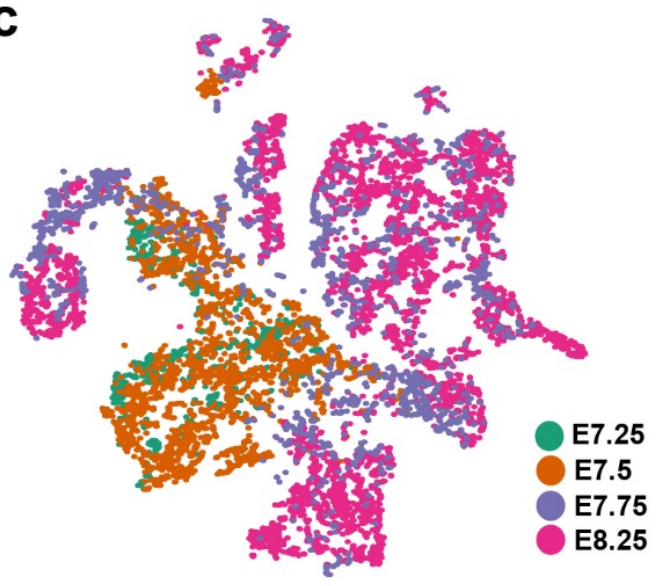

d

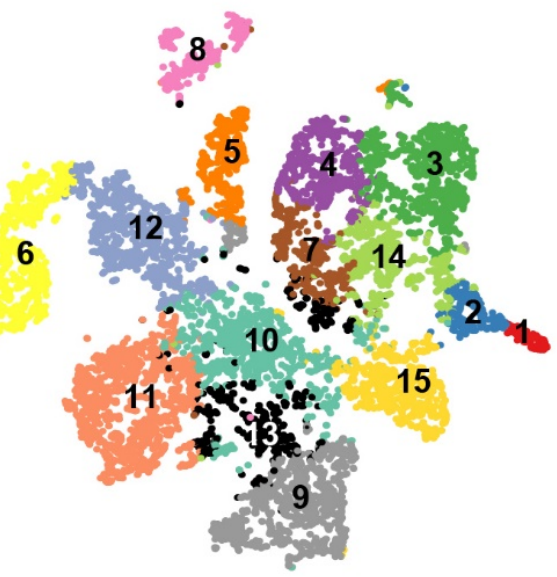

e

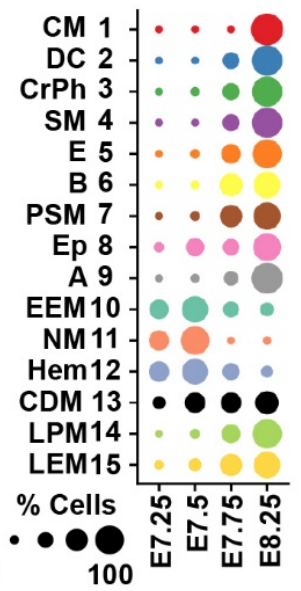

f

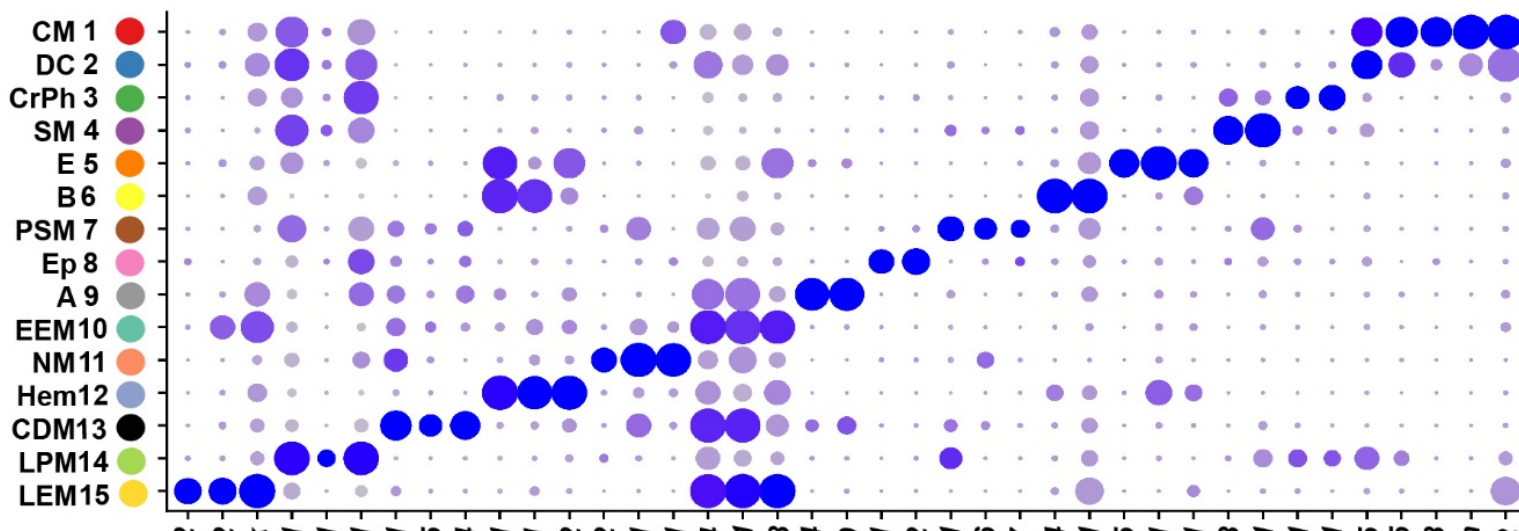

की

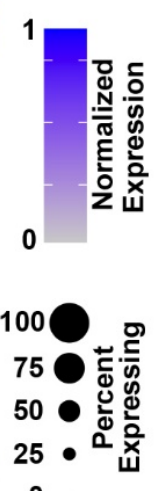

0 . 
752 Figure 1. Mesp1-Cre single-cell maps reveal diverse cell types participating in early

753 mouse mesoderm development. a, Mesp1-Cre scRNA-seq experimental design. Mesp1-

754 Cre; Rosa26-tdT embryos were harvested for scRNA-seq at E7.25 (no bud stage); E7.5 (early

755 bud stage); E7.75 (early head fold stage); and E8.25 (somite stage) as shown in representative

756 bright-field and Mespl-Cre; tdT+ (Mesp1 lineage) micrographs. Illustration below these

757 micrographs shows tissues genetically labeled by Mesp1-Cre in embryos, and workflow for

758 capturing these labeled single cells for RNA sequencing. Scale bars, $150 \mu \mathrm{m}$. b, scRNA-

759 seq data is displayed by tSNE plots at each developmental stage. Cells are colored

760 according to their cell identities in $\mathbf{d}, \mathbf{e}, \mathbf{f} . \mathbf{c}, \mathbf{d}, \mathrm{tSNE}$ plot of scRNA-seq data across all

761 examined stages displays individual cells (single dots) by (c) developmental stages or (d) cell

762 types. e, Dot plot shows distribution of each cell type across different embryonic stages. f,

763 Dot plot of key marker genes identifies each cell cluster. A, Allantois; B, Blood; CDM,

764 Caudal Mesoderm; CrPh, Cranial-pharyngeal mesoderm; CM, Cardiomyocytes; DC,

765 Developing Cardiomyocytes; E, Endothelium; Ep, Epithelium; EEM, Early Extraembryonic

766 Mesoderm; Hem, Hemogenic Mesoderm; HT, Heart tube; LEM, Late Extraembryonic

767 Mesoderm; LPM, Lateral plate mesoderm; NM, Nascent Mesoderm; PSM, Pre-somitic

768 mesoderm; PS, Primitive streak, SM, Somite mesoderm. 


\section{Figure 2}

a

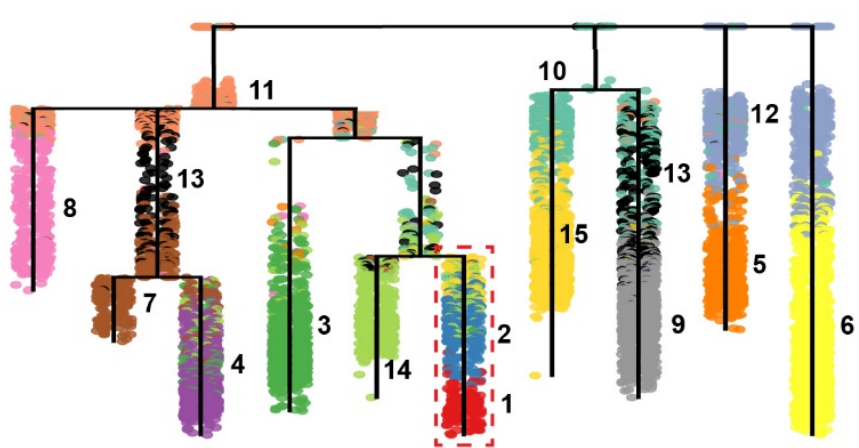

1 Cardiomyocytes (CM)

2 Developing Cardiomyocytes (DC)

3 Cranial Pharyngeal Mesoderm (CrPh)

4 Somite Mesoderm (SM)

5 Endothelium (E)

6 Blood (B)

7 Pre-somitic Mesoderm (PSM)

8 Epithelium (Ep)
9 Allantois (A)

10 Early Extraembryonic

Mesoderm (EEM)

11 Nascent Mesoderm (NM)

12 Hemogenic Mesoderm (Hem)

13 Caudal Mesoderm (CDM)

14 Lateral PlateMesoderm (LPM)

15 Late Extraembryonic Mesoderm (LEM)
C

Cluster

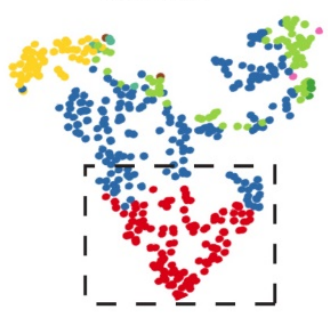

Subcluster

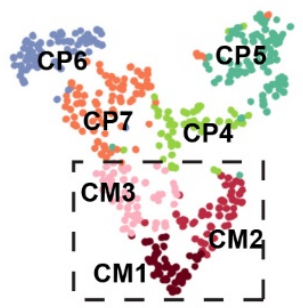

b

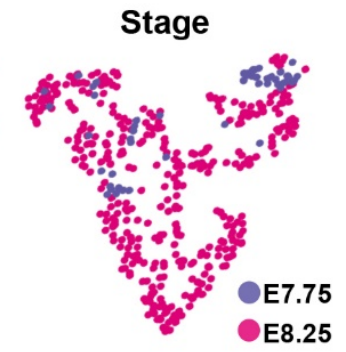

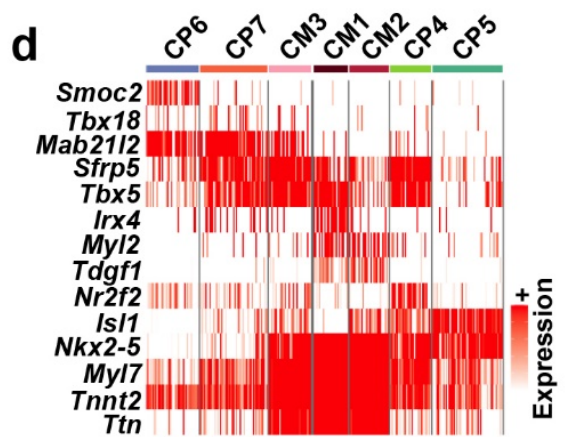

Irx4IMab21/2/Tdgf1
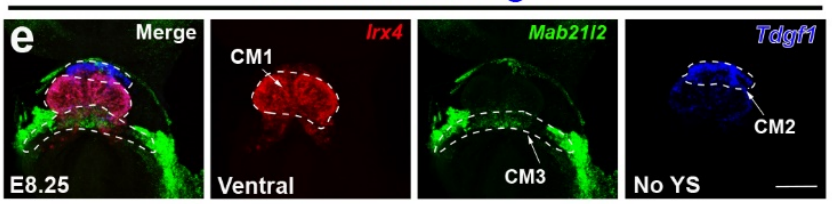

Mab21/2/Nkx2-5/Myl7
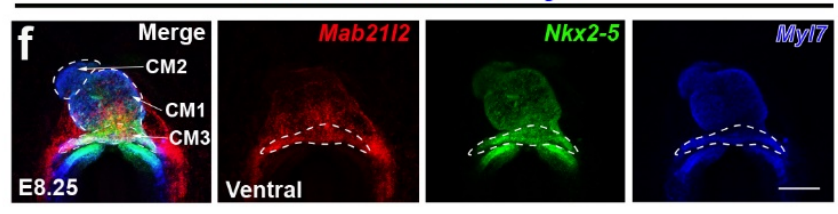

Nr2f2/Sfrp5/Myl7
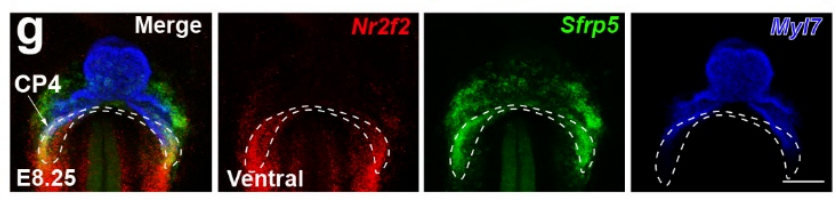

Myl7//s/1/DAPI
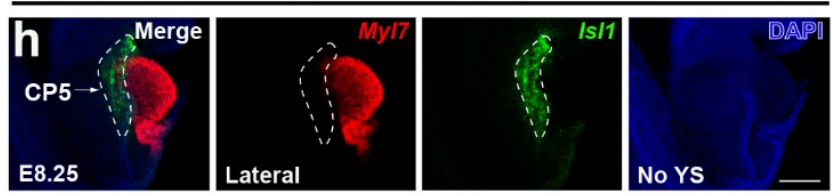

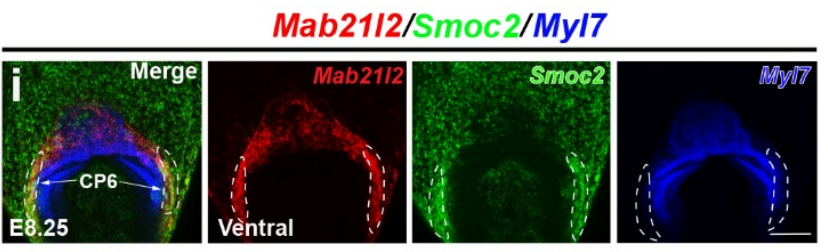

Mab21/2/Sfrp5/Tbx5
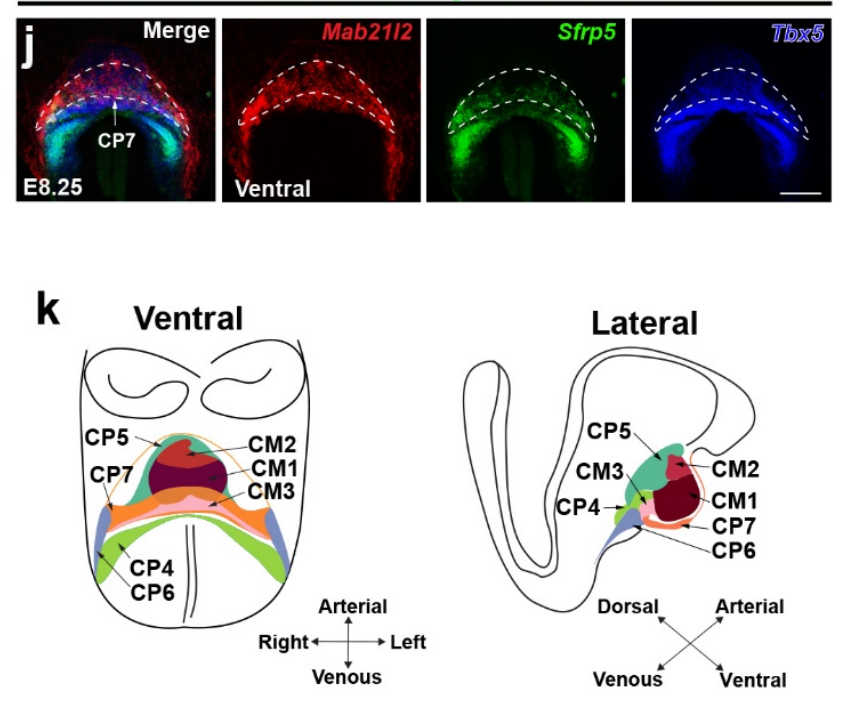
770 Figure 2. Mesp1-Cre scRNA-seq trajectory analysis reconstructs developmental cell

771 lineage trees during mesoderm/heart organogenesis. a, b, URD inferred lineage tree, as

772 displayed by (a) dendogram or (b) force-directed layout, reveals the developmental history of

773 Mesp1 mesoderm-derived organs. Red dashed box in $\mathbf{a}, \mathbf{b}$ outlines cardiomyocyte branch,

774 which is further magnified in $\mathbf{b}$. The magnified cardiomyocyte branch shows that

775 cardiomyocytes may derive from both late extraembryonic mesoderm (LEM) and lateral plate

776 mesoderm (LPM) progenitor cells. c, tSNE layout of cells from only the cardiomyocyte

777 branch (boxed area in $\mathbf{a}, \mathbf{b}$ ) reveals seven cardiac subclusters composing the cardiomyocyte

778 branch including three distinct cardiomyocyte populations (CM1-3) and four specific cardiac

779 progenitor cell-types (CP4-7). d, Heatmap of differentially expressed marker genes

780 identifies each cardiac subcluster. e-j, RNAscope in situ hybridization (ISH) of

781 representative marker genes for each cardiac subcluster cell population shows their location

782 in E8.25 embryos. $\mathrm{n}=3$ per panel. Scale bars, $100 \mu \mathrm{m}$. The extraembryonic tissue and

783 part of the pericardium tissue were removed in $\mathbf{e}, \mathbf{h}$ to show the underlying heart tube. $\mathbf{k}$,

784 Diagram illustrates the seven different cardiac subclusters in an E8.25 embryo. 


\section{Figure 3}

a

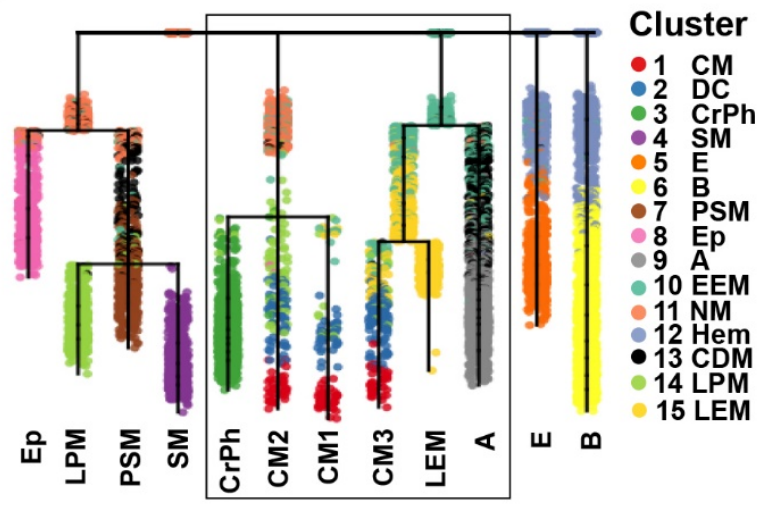

b

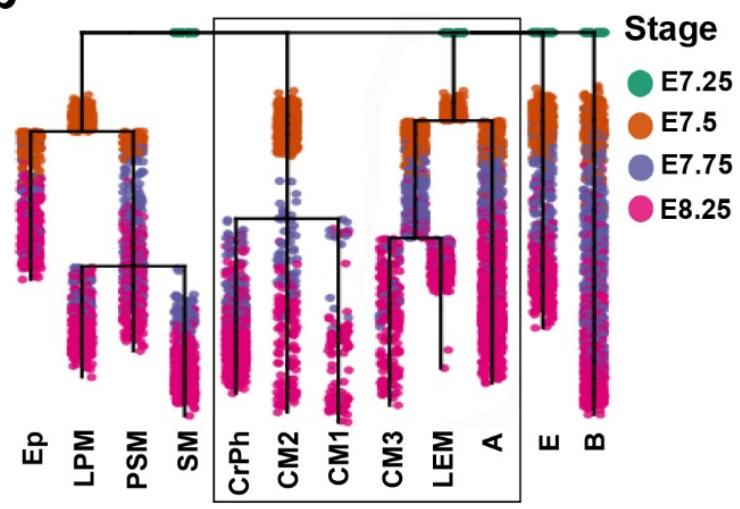

C

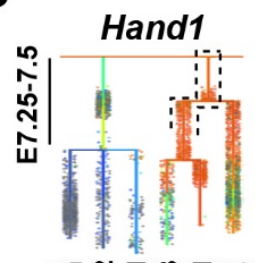

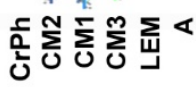

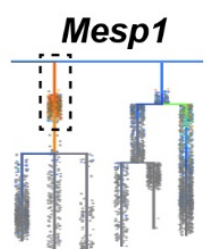

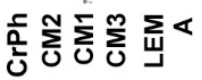

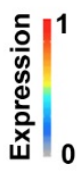

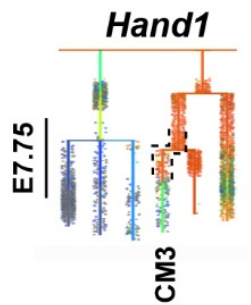

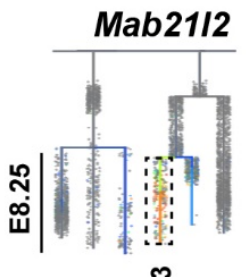

$\sum_{U}^{m}$

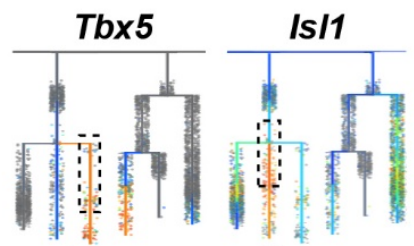

ญั
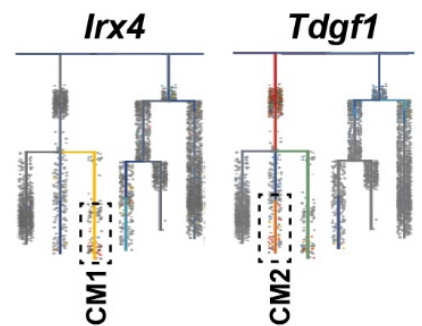
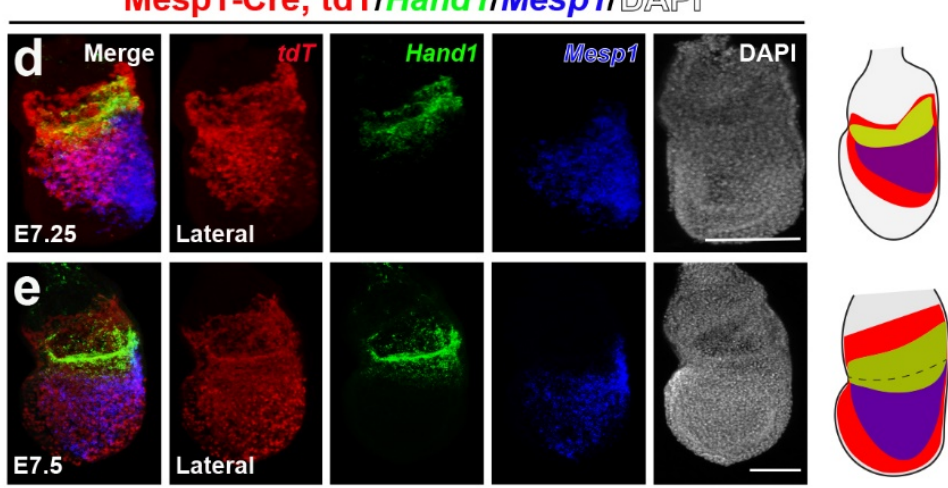

Tbx5IHand1/Is/1/DAPQ
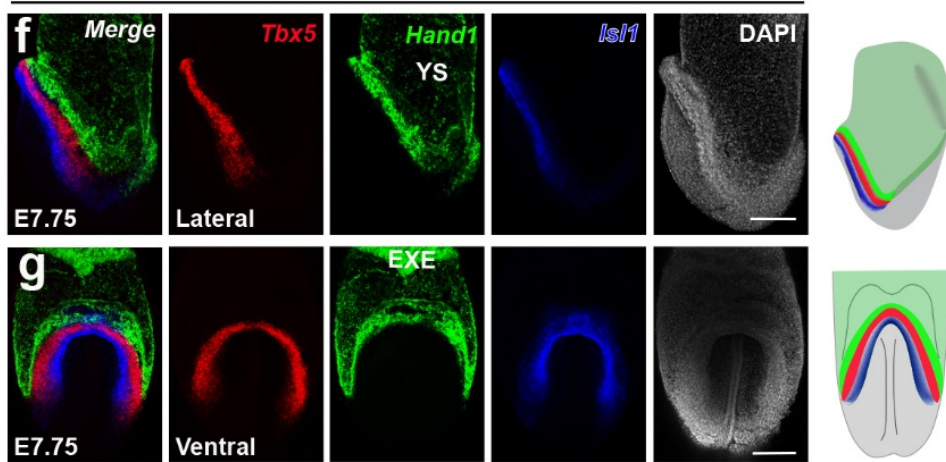
786 Figure 3. Distinct cardiomyocyte lineages derive from intra- and extra-embryonic

787 related developmental origins. a, b, Reconstructed URD developmental cell lineage trees

788 using the three distinct subclustered cardiomyocyte populations predict that CM1/CM2 and

789 CM3 cardiomyocytes derive respectively from intra- and extra-embryonic related progenitor

790 sources, as displayed by (a) cell type and (b) developmental stages. The cardiomyocyte-

791 related branches of the URD developmental tree are outlined with box. c, Marker genes

792 differentially expressed among the lineages for each cardiomyocyte subcluster are plotted on

793 the URD cardiomyocyte-related branches. Hand1 and Mab21l2 mark early and late regions

794 of the CM3 lineage, respectively. Mesp1, Tbx5, Isl1, Irx4 and Tdgf1 label different regions

795 of the CM1 and CM2 lineage branches. d, e, RNAscope in situ hybridization (ISH) of

796 Mespl and Hand1 was performed in (d) E7.25 and (e) E7.5 Mesp1-Cre; Rosa26-tdT

797 embryos. The diagram illustrates both the gene expression pattern of Hand1 and Mespl and

798 Mesp1-Cre lineage-traced cells in these embryos. f, g, RNAscope ISH of Hand1, Tbx5, and

799 Isl1 was performed in E7.75 embryos. The diagram illustrates the expression pattern of

800 Handl, Tbx5, and Isll in these embryos. $\mathrm{n}=3$ per panel. Scale bars, $100 \mu \mathrm{m}$. EXE,

801 Extraembryonic Ectoderm; YS, Yolk Sac. 


\section{Figure 4}
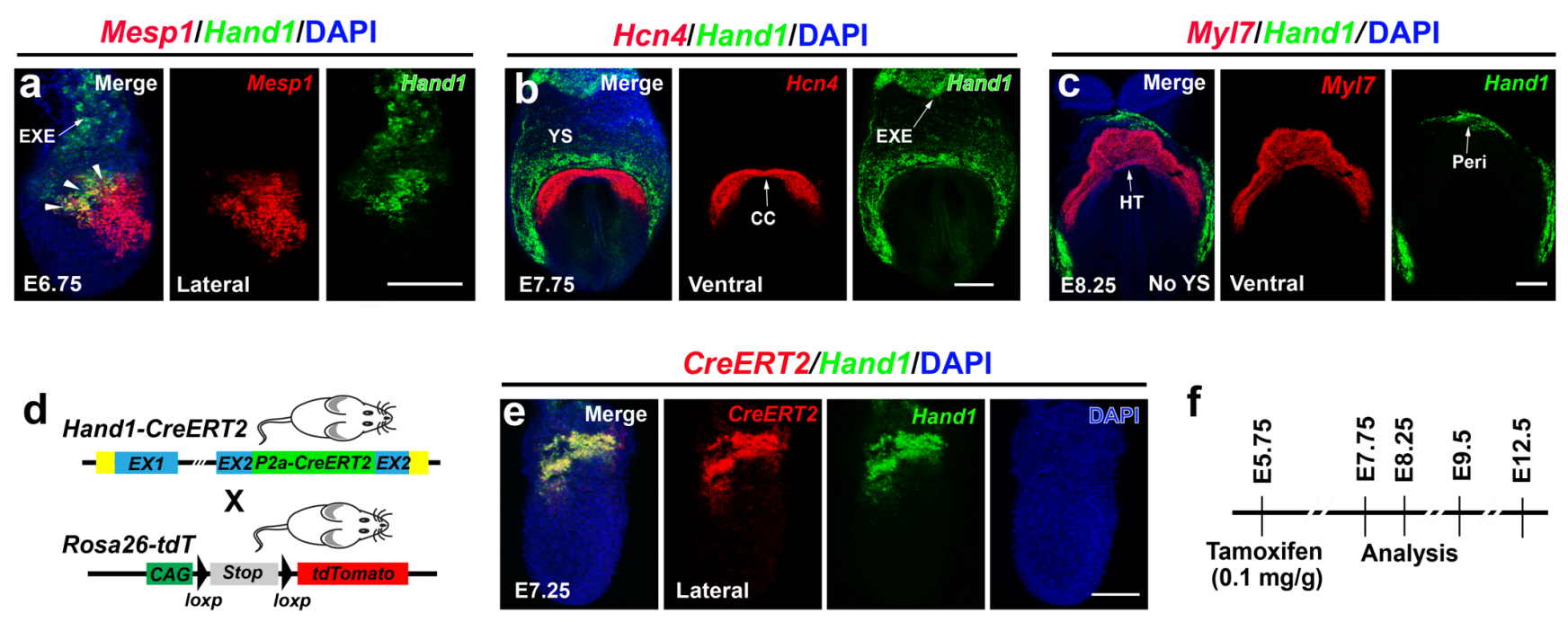

tdTIHen4IDAPI
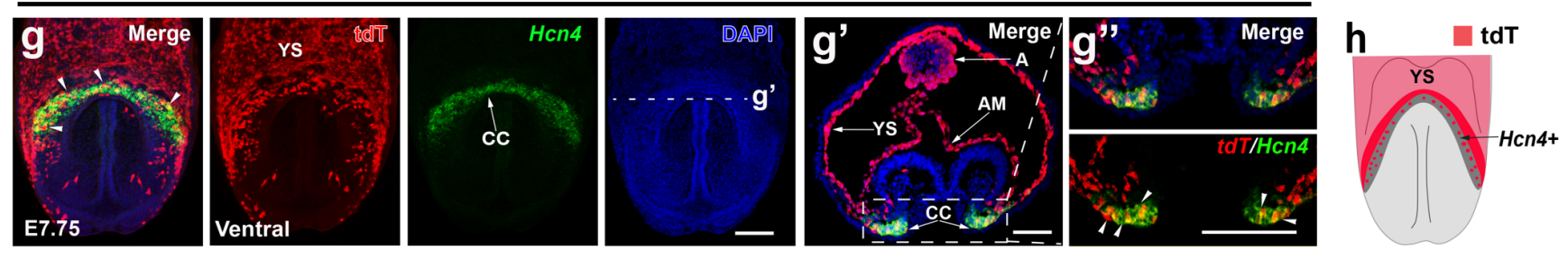

tdT/My/7/DAPI
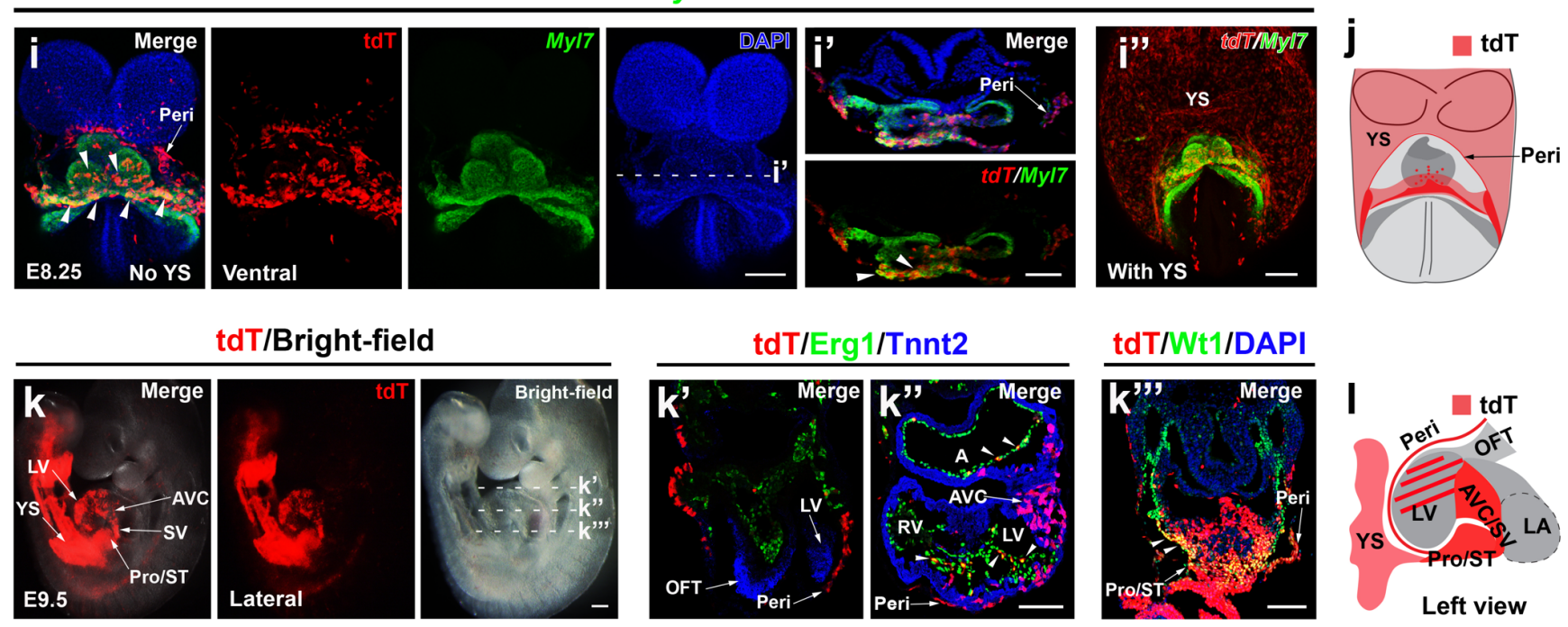

tdT/Bright-field
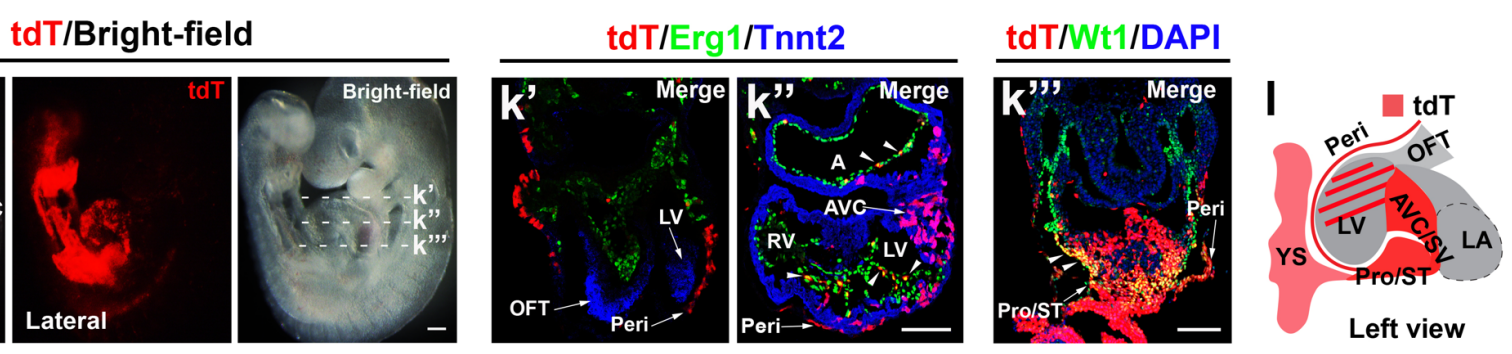

\section{tdT/Bright-field}
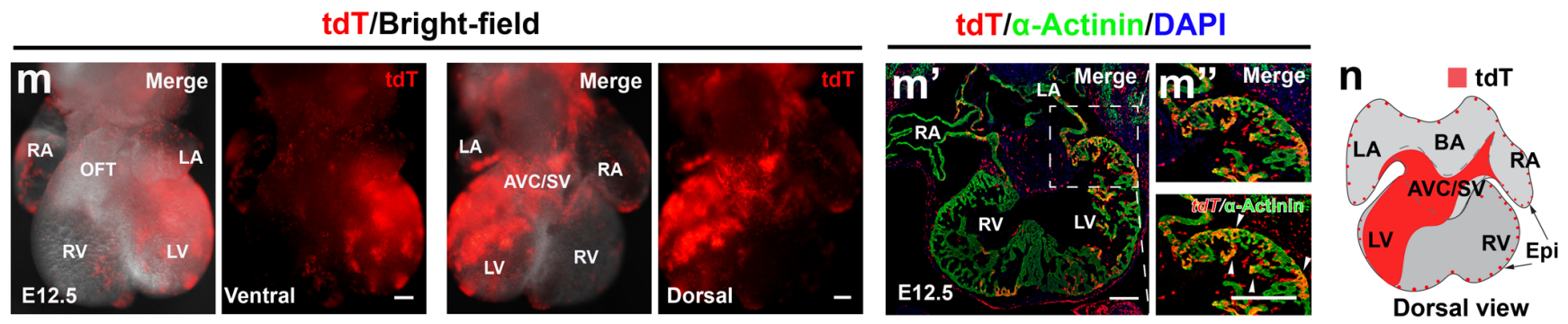
803 Figure 4. Lineage tracing studies reveal that early gastrulating Hand1+ cells

804

805

806

807

808

809

810

811 contribute to not only a distinct subpopulation of first heart lineage cardiomyocytes but also serosal mesothelial lineages (pericardial, epicardial cells) in the heart. a-c, RNAscope in situ hybridization (ISH) reveals that Handl is expressed with (a) Mespl at the embryonic and extraembryonic boundary in E6.75 embryos (arrowheads), (b) dorso-laterally around the cardiac crescent as detected by Hcn4 at E7.75, and (c) in the pericardium which overlays the heart tube (HT) as detected by Myl7 at E8.25. The yolk sac and part of the pericardium were removed in $\mathbf{c}$ to show the underlying heart tube. $\mathbf{d}-\mathbf{n}$, Lineage tracing studies using Hand1-CreERT2 and Rosa26-tdT mice (shown in d) map the fate of early gastrulating Hand1+ cells. e, RNAscope ISH in Hand1-CreERT2 embryos shows that expression of CreERT2 precisely recapitulates the expression of Hand1. f, Schematic outlines the experimental strategy for Hand1-CreERT2 genetic fate mapping studies shown in g-n. Tamoxifen was given at E5.75, and embryos were examined for Hand1-CreERT2 genetically-labeled tdT+ cells at E7.75, E8.25, E9.5 and E12.5. g-n, RNAscope ISH and immunohistochemistry of whole mount and cross sections of these embryos reveal the contribution of Hand1-CreERT2 genetically-labeled tdT+ cells at (g) E7.75, (i) E8.25, (k) E9.5 and (m) E12.5. g', i', k', k', k',', Insets show transverse sections of $\mathbf{g}, \mathbf{i}, \mathbf{k}$ at indicated dashed lines, respectively. m', Inset shows coronal section of $\mathbf{m} . \mathbf{g}$ g”, m', Insets are magnification of $\mathbf{g}$ ', m' boxed area. Arrowheads point to tdT + cells expressing $(\mathbf{g}, \mathbf{g}$ '’) Hcn4, (i, i') Myl7, (k'") Erg1, (k',') Wt1 and (m'") $\alpha$-Actinin. h, j, l, n, Diagrams summarize the anatomical location of Hand1-CreERT2 genetically-labeled tdT + cells at the embryonic stages analyzed. $\mathrm{n}=3$ embryos for each stage. Scale bars, $100 \mu \mathrm{m}$. AM, Amnion; AVC, Atrioventricular Canal; BA, Base of the Atrium; CC, Cardiac Crescent; Epi, Epicardium; EXE, Extraembryonic Ectoderm; HT, Heart tube; LA, Left Atrium; LV, Left 
827 Ventricle; OFT, Outflow Tract; Peri, Pericardium; Pro, Proepicardium; RA, Right Atrium;

828 RV, Right Ventricle; SV, Sinus Venosus; ST, Septum transversum; YS, Yolk Sac. 


\section{Figure 5}

a

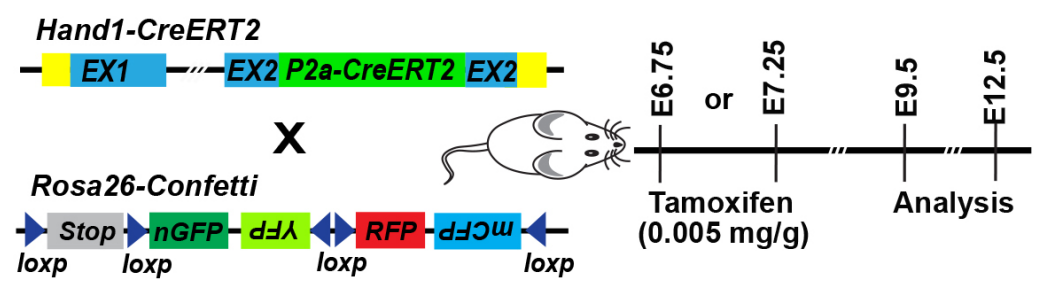

b

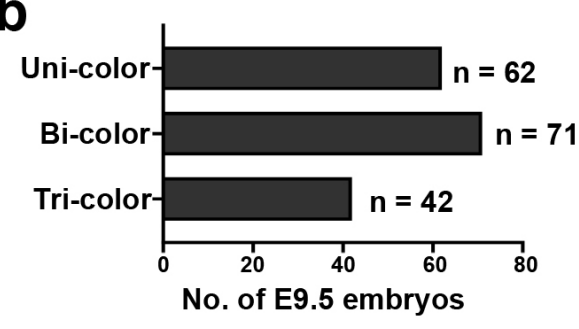

C

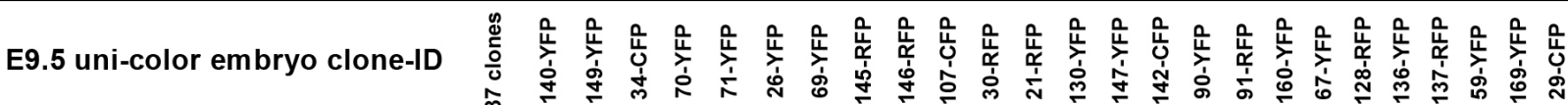

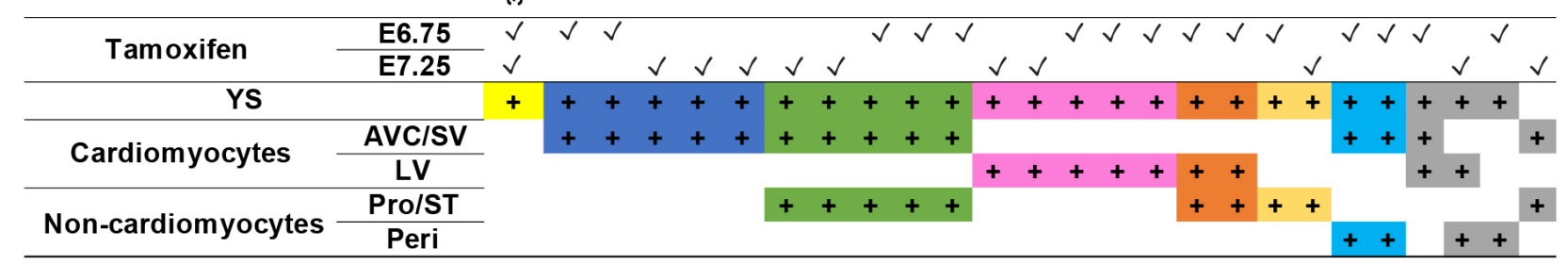

d

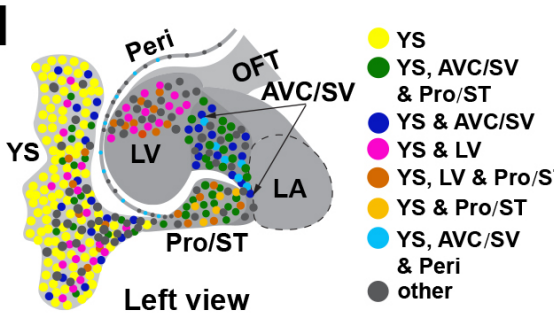

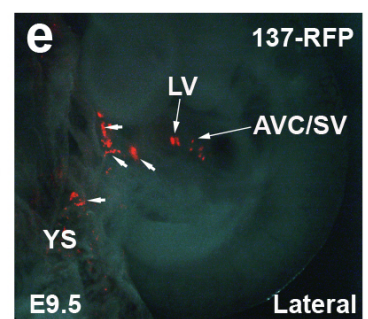

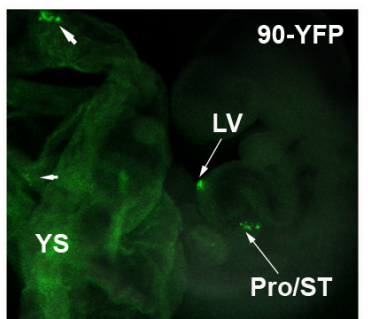

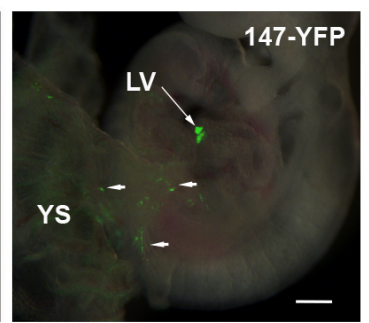

$\mathbf{f}$

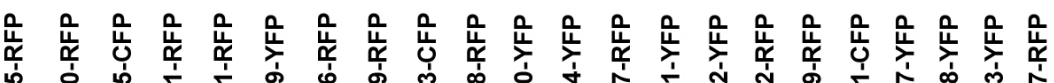

E12.5 uni-color heart clone-ID

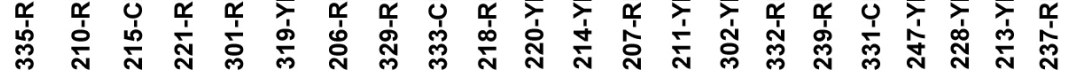

\begin{tabular}{|c|c|c|c|c|c|c|c|c|c|c|c|c|c|c|c|c|c|c|c|c|}
\hline \multirow{2}{*}{ Tamoxifen } & E6.75 & $\checkmark$ & & & $\checkmark$ & $\checkmark$ & & $\checkmark \quad \checkmark$ & & & & & & $\checkmark \checkmark$ & & & $\checkmark$ & & $\checkmark$ & \\
\hline & E7.25 & & $\checkmark \checkmark$ & $\checkmark$ & & & $\checkmark$ & & $\checkmark$ & $\checkmark$ & $\checkmark$ & $\checkmark$ & $\checkmark$ & & $\checkmark$ & & & & & $\checkmark$ \\
\hline \multirow{3}{*}{ Cardiomyocytes } & AVC/SV & + & $+\quad+$ & + & + & + & + & & & & & & & & & & & & + & + \\
\hline & LV & & & & & & & $+\quad+$ & + & + & + & + & + & ++ & & & & & + & \\
\hline & RA & & & & & & & & & & & & & & & & & & & + \\
\hline Non-cardiomyocytes & Epi & & & & + & + & + & $+\quad+$ & + & + & + & & & & + & + & + & & & \\
\hline
\end{tabular}
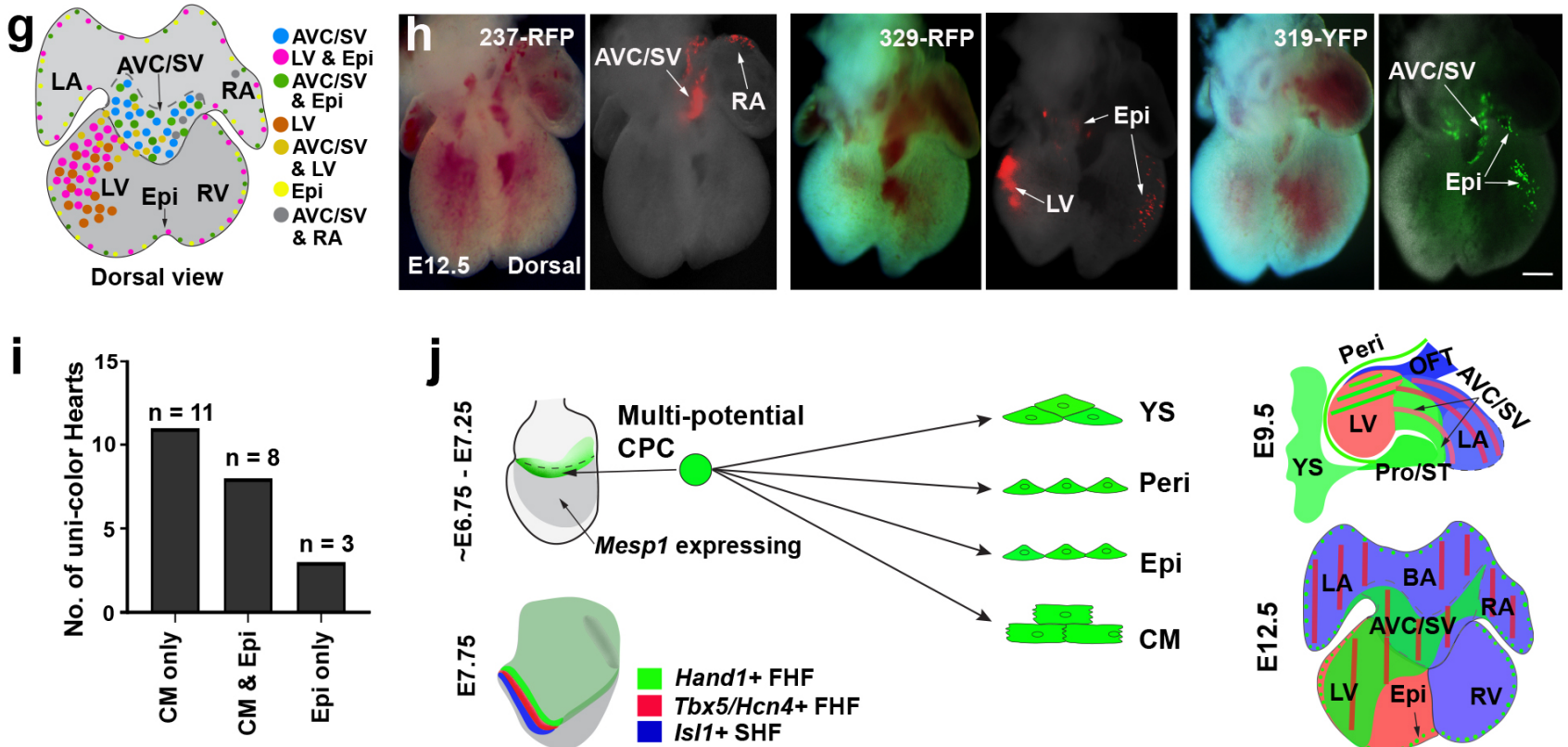

Hand1+ FHF

Tbx5/Hcn4+ FHF

IsI1+ SHF

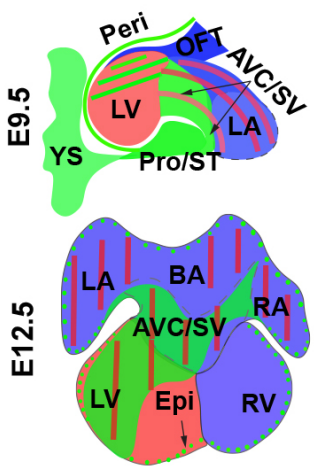


830 Figure 5. Clonal analysis reveals multipotentiality in early Hand1+ progenitors.

831 a, Schematic outlines experimental strategy for Hand1-CreERT2; Rosa26-Confetti clonal

832 analyses. b, Bar graph displays the number of uni-color, bi-color or tri-color Hand1-

833 CreERT2; Rosa26-Confetti embryos at E9.5. c, f, Clonal analyses of uni-color (c) E9.5 and

834 (f) E12.5 embryos reveal that individual Hand1-CreERT2; Rosa26-Confetti clones labeled at

835 E6.75 or E7.25 have the capacity to generate multiple cell types that can contribute to the

836 yolk sac and/or heart. $\quad \mathbf{d}, \mathbf{g}$, Diagram summarizes the contribution of Hand1-CreERT2;

837 Rosa26-Confetti genetically-labeled clones in the heart and yolk sac at (d) E9.5 and in the

838 heart at (g) E12.5. e, h, Representative (e) E9.5 and (h) E12.5 uni-color embryos show

839 individual Hand1-CreERT2; Rosa26-Confetti genetically-labeled clones contributing to

840 different combinations of tissues and cell types: (e) AVC/SV, LV and YS (clone \#137-RFP);

841 LV, Pro/ST and YS (clone \#90-YFP); LV and YS (clone \#147-YFP); (h) AVC/SV and RA

842 (clone \# 237-RFP); LV and Epi (clone \# 329-RFP); the AVC/SV and Epi (clone \# 319-YFP).

843 Arrowheads point to yolk sac cells in e. Scale bars, $200 \mu \mathrm{m}$. i, Bar graph displays the

844 number of uni-color E12.5 hearts with clones contributing to cardiomyocytes only, epicardial

845 cells and cardiomyocytes, or only epicardial cells. j, Model summarizes the

846 multipotentiality of Hand1 + cardiac progenitor cells (CPC) between E6.75 - E7.25 in relation

847 to the contribution of reported FHF/SHF progenitors. AVC, Atrioventricular Canal; BA,

848 Base of the Atrium; CM, cardiomyocytes; Epi, Epicardium; LA, Left Atrium; LV, Left

849 Ventricle; OFT, Outflow Tract; Peri, Pericardium; Pro, Proepicardium; RA, Right Atrium;

850 RV, Right Ventricle; SV, Sinus Venosus; ST, Septum Transversum; YS, Yolk Sac. 


\section{Extended Data Figure 1}

a

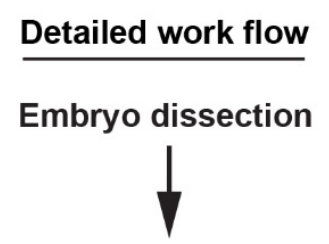

Single-cell dissociation

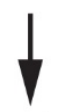

Enrichment for single Mesp1 lineage cells via FACS<smiles>C1CCC2(CC1)CCCC2</smiles>

Capture individual Mesp1 lineage cells via 10X microfluidics

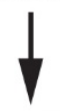

Barcode addition and 3'-end cDNA library, QC analysis<smiles>CC(C)(C)C1(C)CCCCC1</smiles>

QC analysis and identify \# of cells captured per sample: Shallow sequencing

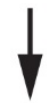

Deep sequencing

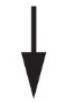

QC analysis and normalization

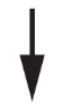

Identify similar cells and \# of clusters: tSNE and silhouette analysis b

\begin{tabular}{|c|c|c|c|c|}
\hline Stage & $\begin{array}{c}\text { Time of } \\
\text { dissection }\end{array}$ & $\begin{array}{c}\text { \# of embryos } \\
\text { per stage }\end{array}$ & $\begin{array}{c}\text { \# of } \\
\text { sequencing } \\
\text { batches }\end{array}$ & $\begin{array}{l}\text { \# of cells } \\
\text { captured }\end{array}$ \\
\hline No Bud (OB) & E7.25 & 3 & 1 & 903 \\
\hline Late Bud (LB) & E7.5 & 12 & 5 & 2401 \\
\hline Early Head Fold (EHF) & E7.75 & 2 & 2 & 2226 \\
\hline Somite (1-4) & E8.25 & 5 & 3 & 4977 \\
\hline \multicolumn{2}{|c|}{ Total \# of cells captured } & \multicolumn{3}{|l|}{10507} \\
\hline \multicolumn{2}{|c|}{ Total \# of cell analyzed (after QC) } & \multicolumn{3}{|l|}{9072} \\
\hline \multicolumn{2}{|c|}{ Average UMI per cell } & \multicolumn{3}{|l|}{60450} \\
\hline \multicolumn{2}{|c|}{ Average \# of genes per cell } & \multicolumn{3}{|l|}{6062} \\
\hline \multicolumn{2}{|c|}{ Average sequence saturation } & \multicolumn{3}{|l|}{$65.7 \%$} \\
\hline
\end{tabular}

C tSNE plots of sequencing batches
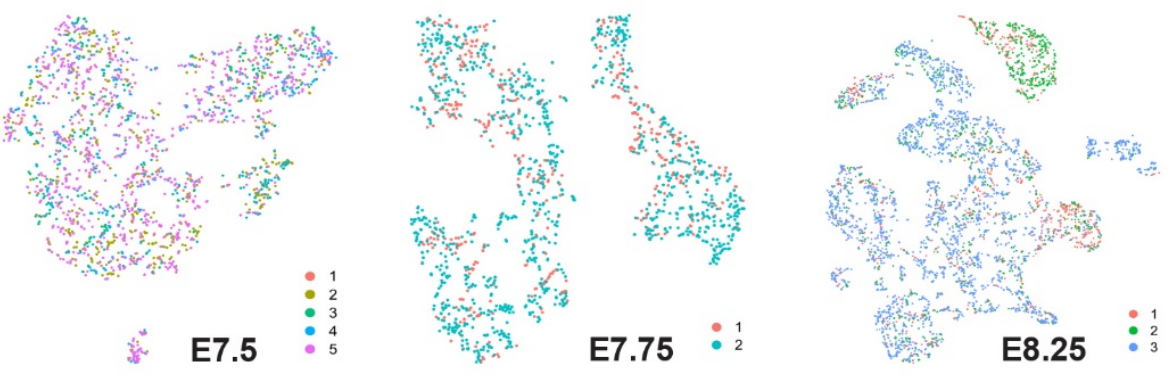

d

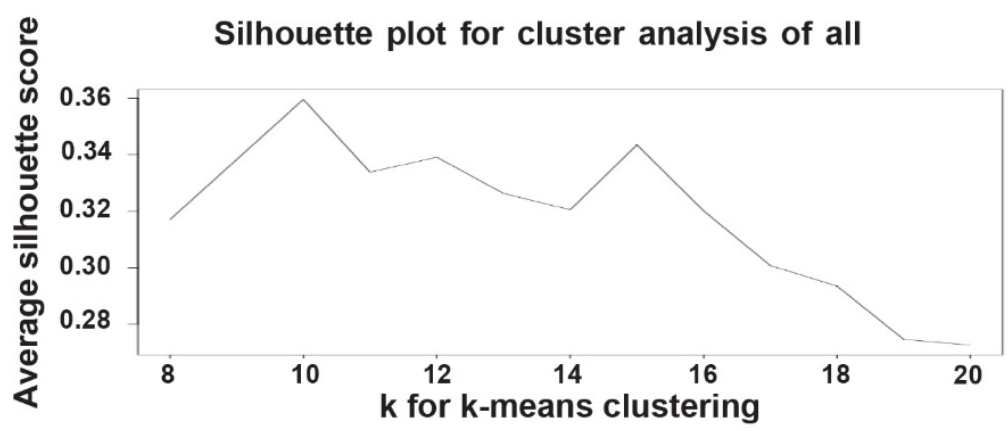

e \# of cells per cluster per stage

\begin{tabular}{|c|c|c|c|c|c|c|c|c|c|c|c|c|c|c|c|c|}
\hline & Cluster & 1 & 2 & 3 & 4 & 5 & 6 & 7 & 8 & 9 & 10 & 11 & 12 & 13 & 14 & 15 \\
\hline \multirow{4}{*}{$\begin{array}{l}\stackrel{0}{\pi} \\
\text { ஸे }\end{array}$} & E7.25 & & & & & & & & 5 & & 206 & 304 & 246 & 21 & & \\
\hline & E7.5 & & & 2 & & 2 & 1 & 2 & 61 & 2 & 516 & 843 & 498 & 135 & 1 & 29 \\
\hline & E7.75 & & 26 & 120 & 73 & 93 & 310 & 132 & 43 & 77 & 101 & & 133 & 137 & 109 & 227 \\
\hline & E8.25 & 113 & 169 & 685 & 434 & 311 & 482 & 238 & 225 & 893 & 54 & 1 & 25 & 202 & 397 & 382 \\
\hline
\end{tabular}


852 Extended Data Figure 1. Mesp1-Cre scRNA-seq experimental details show the

853 sequencing, quality control and analysis parameters used on single mesoderm cells. a,

854 Diagram outlines details of the scRNA-seq workflow. b, Table shows the number of

855 embryos and cells harvested and studied at each experimental condition. c, tSNE plots of

856 sequencing experiments show that scRNA-seq datasets are well mixed at each developmental

857 stage with no appreciable batch effects. d, Average silhouette score shows how the number

858 of clusters for scRNA-seq data was determined. e, Table shows the number of cells

859 sequenced and analyzed in each identified cluster at specific stages. 


\section{Extended Data Figure 2}
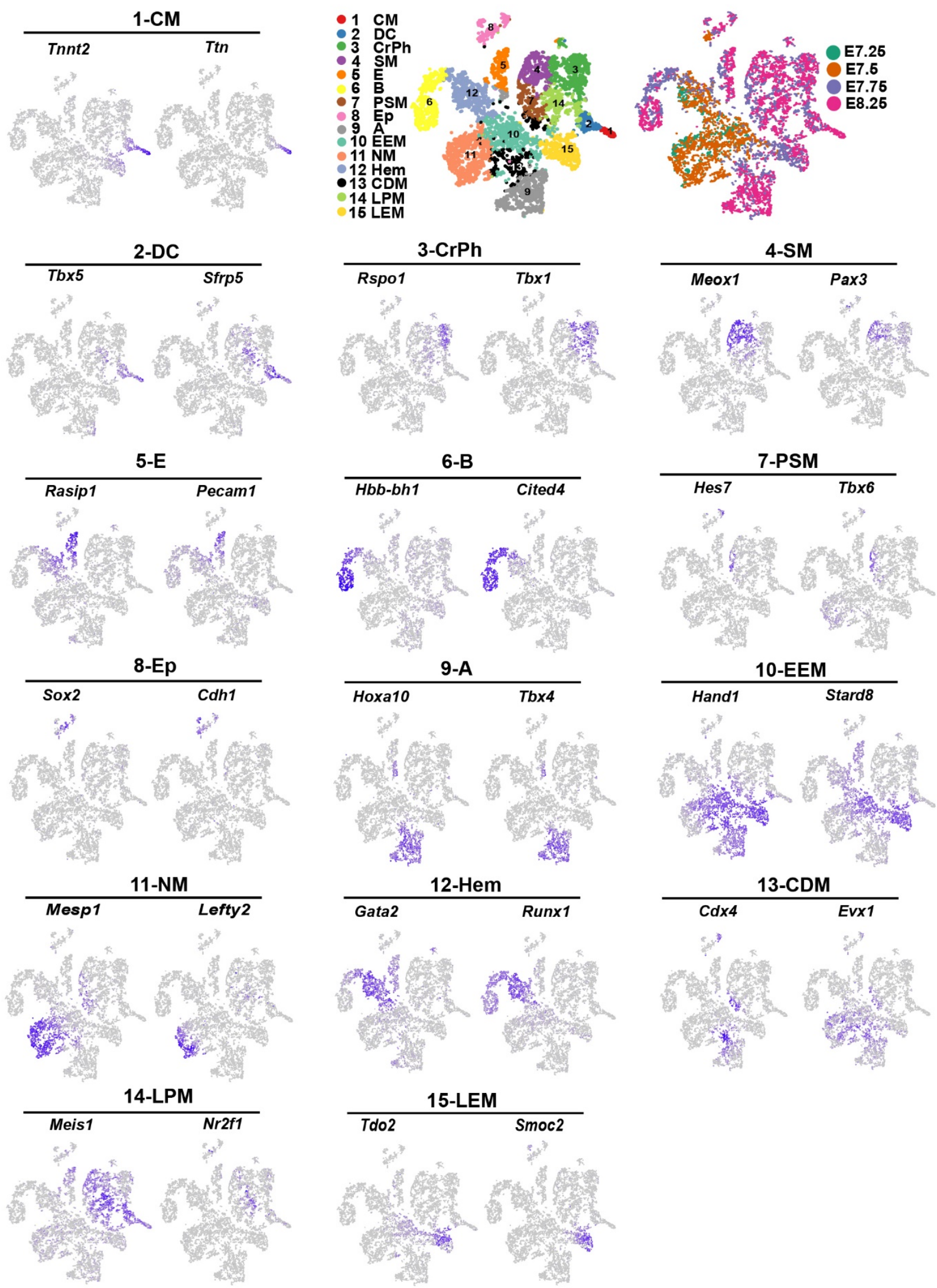
861 Extended Data Figure 2. The expression of gene markers specific to identified clusters

862 is visualized in tSNE plots of Mesp1-Cre scRNA-seq data. The expression of two

863 representative markers for each cluster are displayed on the single-cell tSNE layout of all

864 developmental stages. 


\section{Extended Data Figure 3}

a

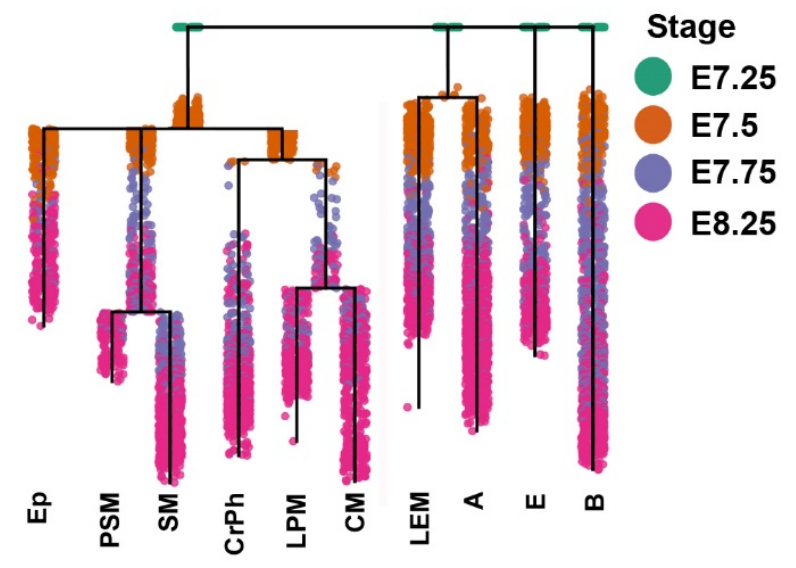

C

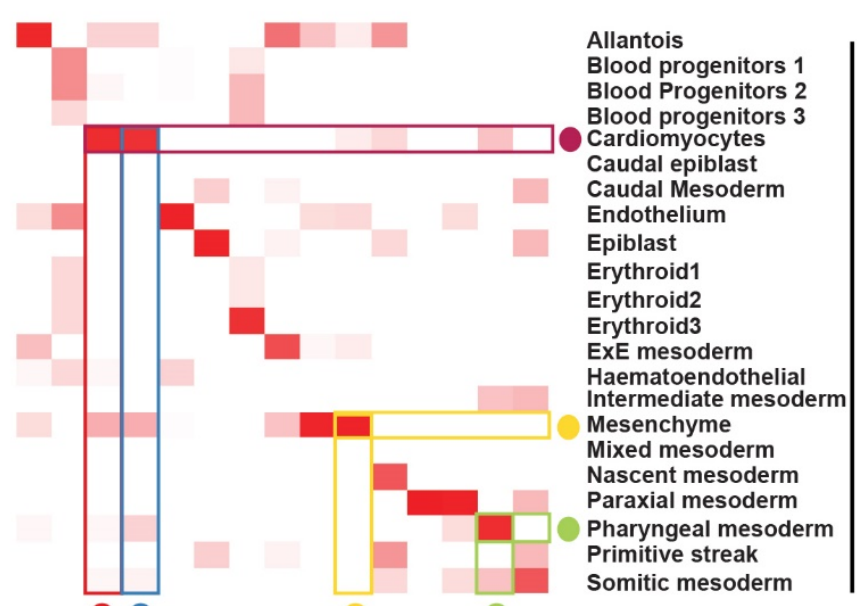

《 b
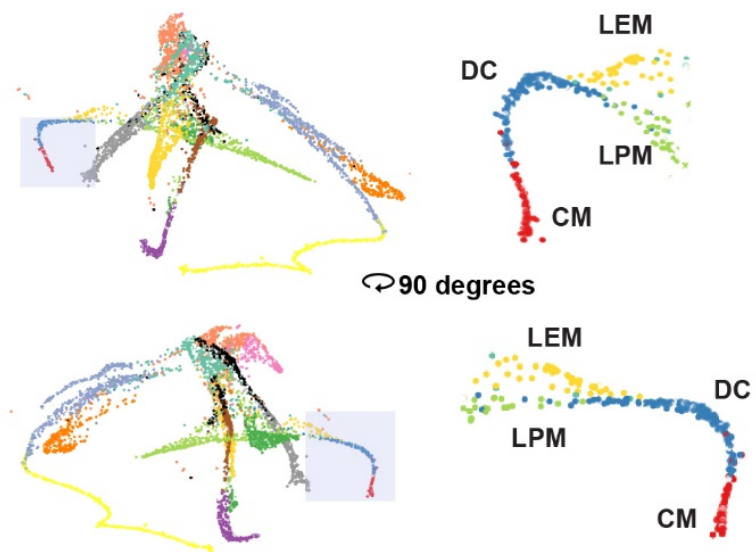

d

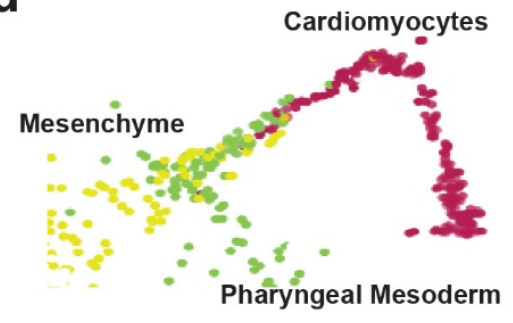

Whole embryo scRNA-seq data (Pijuan-Sala et al)

e

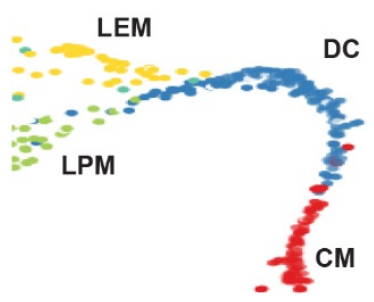

Mesp1 lineage scRNA-seq data 


\section{Extended Data Figure 3. Trajectory analysis in an independent developing mouse}

867 embryo scRNA-seq dataset predicts similar intra- and extra-embryonic related

868 developmental sources of cardiac progenitors. a, Developmental stage of each RNA-

869 sequenced Mesp1-Cre labeled cell was projected onto Mesp1-Cre URD developmental

870 lineage tree reconstructed in Figure. 2a. b, 3D rotations of the force-directed Mesp 1-Cre

871 URD layout provide different views of late extraembryonic mesoderm (LEM) and lateral

872 plate mesoderm (LPM) trajectories converging to form the cardiomyocyte branch. Shaded

873 branches are magnified and shown to the right. c, Heatmap of identified cell-type clusters

874 from Mesp1-Cre and independent mouse embryonic scRNA-seq datasets ${ }^{27}$ reveals

875 corresponding analogous cell-type populations between the datasets. d, e, An URD-derived

876 force-directed layout of these independent scRNA-seq datasets shows how their analogous

877 cell types exhibit similar converging trajectories that create cardiomyocytes. 


\section{Extended Data Figure 4}

a

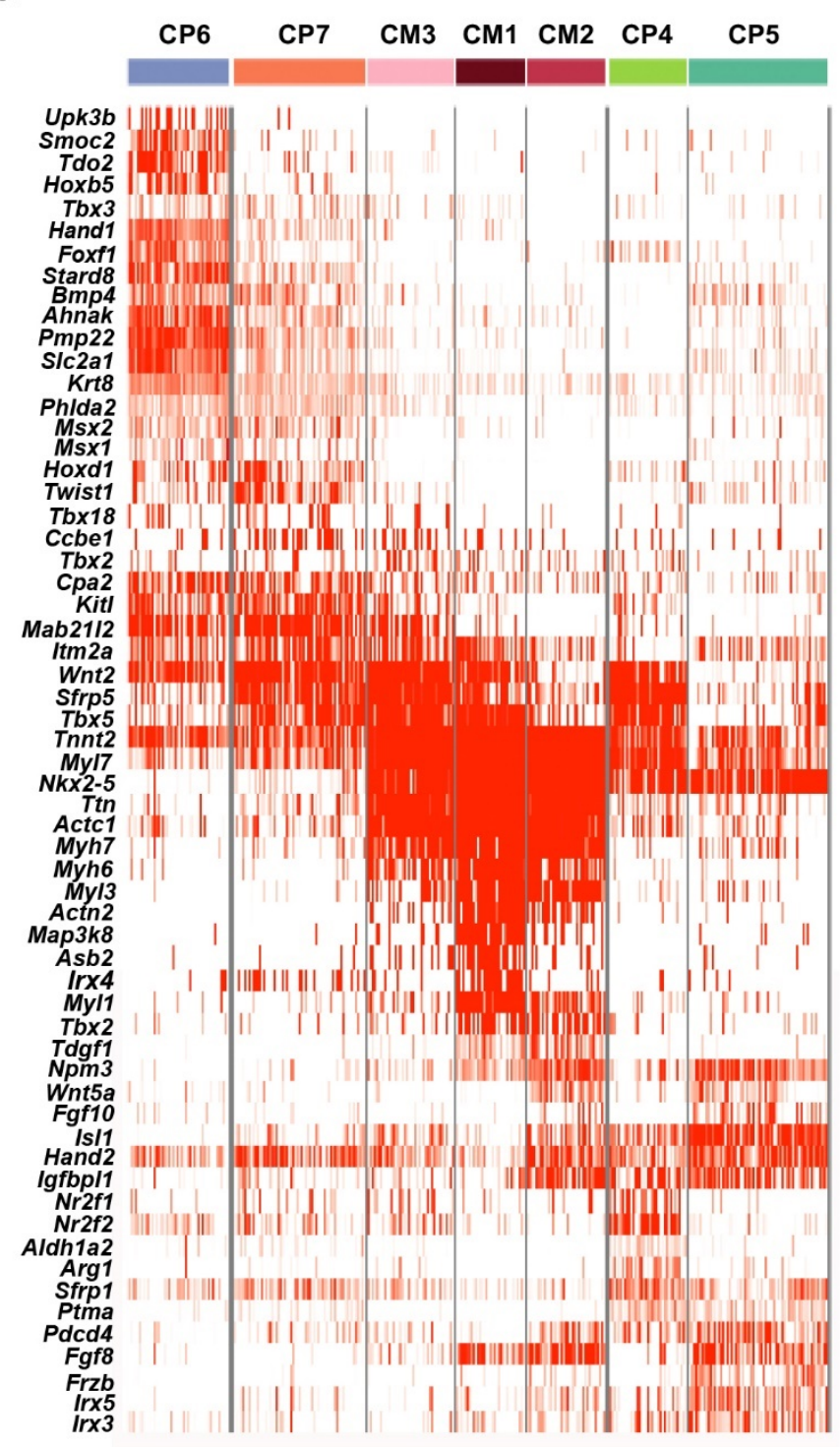

b
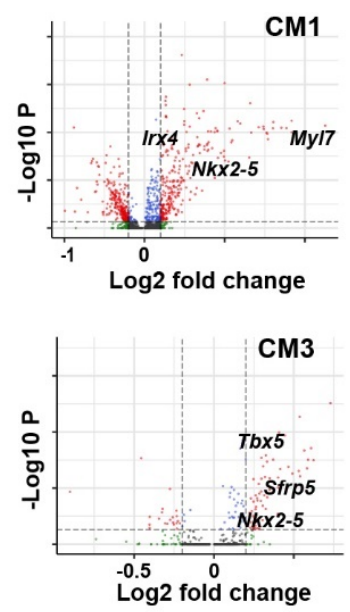

C
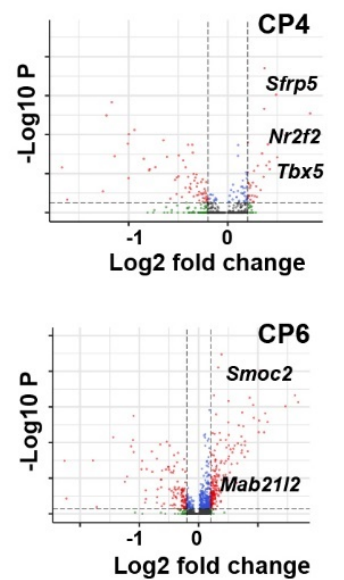

d

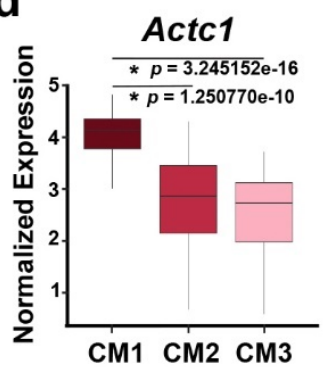

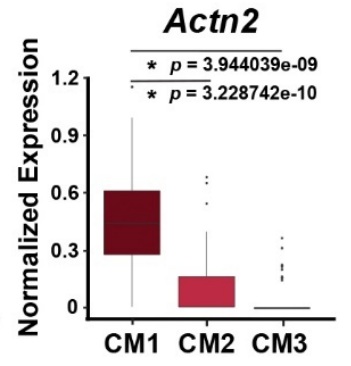
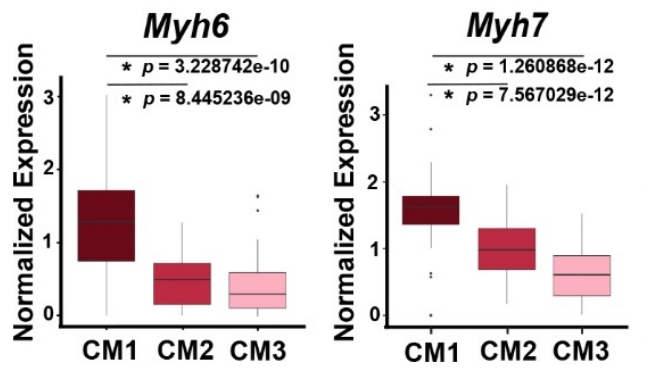
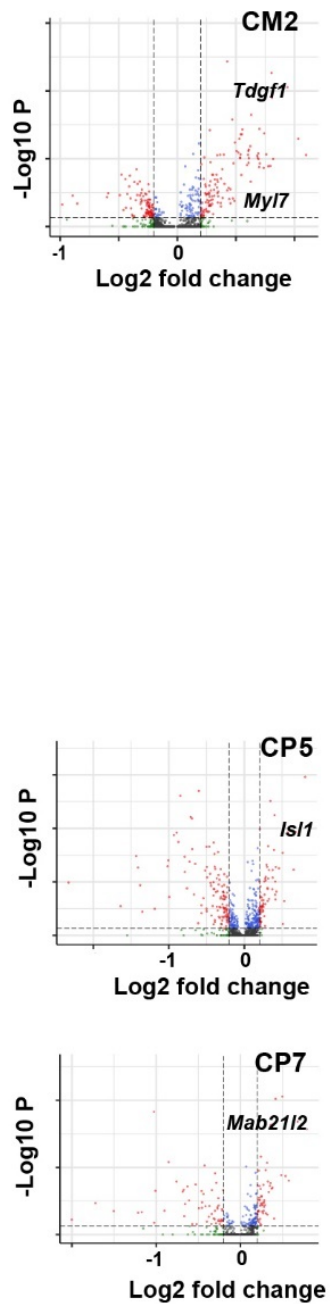

55

s11

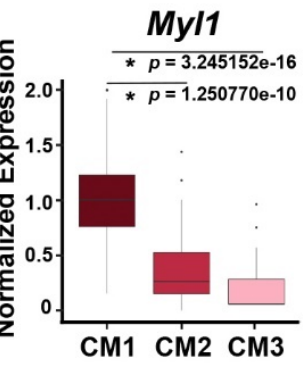


879 Extended Data Figure 4. Differential gene expression analysis reveals cardiac subtype

880 identities and transcriptional profiles. a, An extended heatmap of differentially expressed

881 genes among cardiac subclusters reveals distinct transcriptional profiles for each

882 developmental cardiac subcluster cell type derived in Figure 2c. b, c, Volcano plots

883 summarize the differential expression of each cardiac subcluster (b, cardiomyocyte

884 subclusters and $\mathbf{c}$, cardiac progenitor subclusters) compared to all other cardiac subclusters.

885 d, Box plots show that CM1 cells display higher expression of Actc1, Actn2, Myh6, Myh7 and

886 Myll than that observed in CM2 and CM3. Median, $25^{\text {th }}$ and $75^{\text {th }}$ quartile, and extreme

887 values within 1.5 times the interquartile range are indicated by the center line, bottom and top

888 of the box, and ends of whiskers, respectively. Outliers outside 1.5 times the interquartile

889 range appear as individual points. $* p<0.01$ by Wilcoxon rank sum test. 


\section{Extended Data Figure 5}

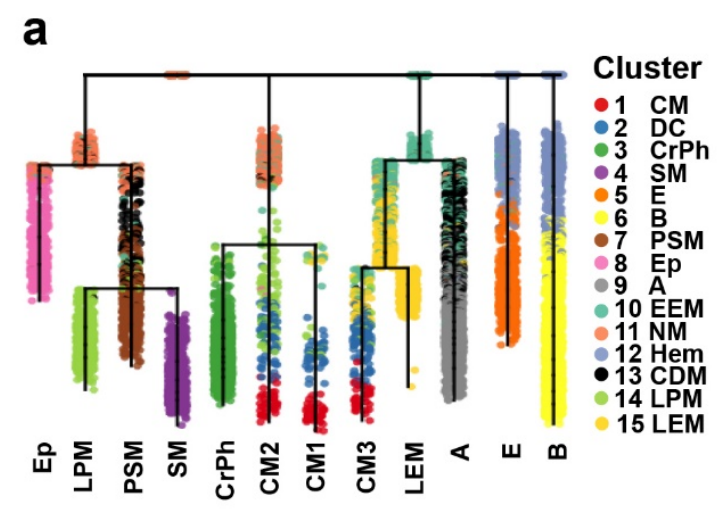

b
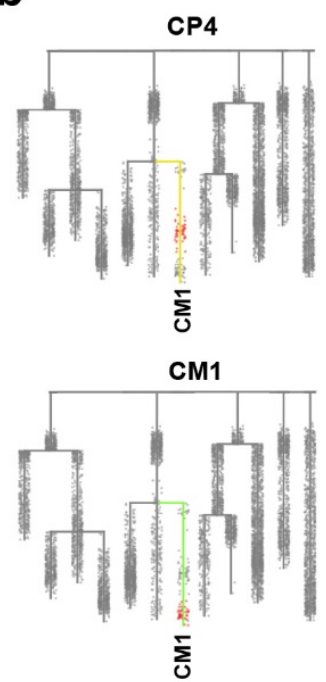

C
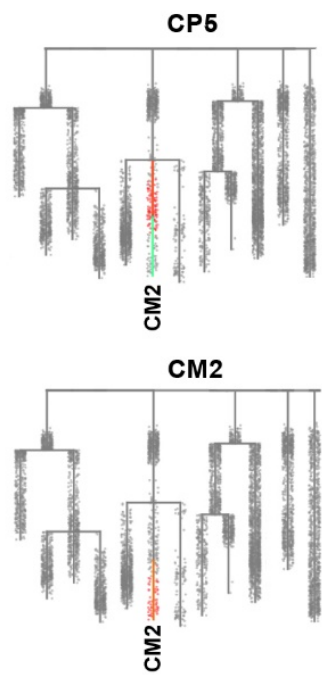

d
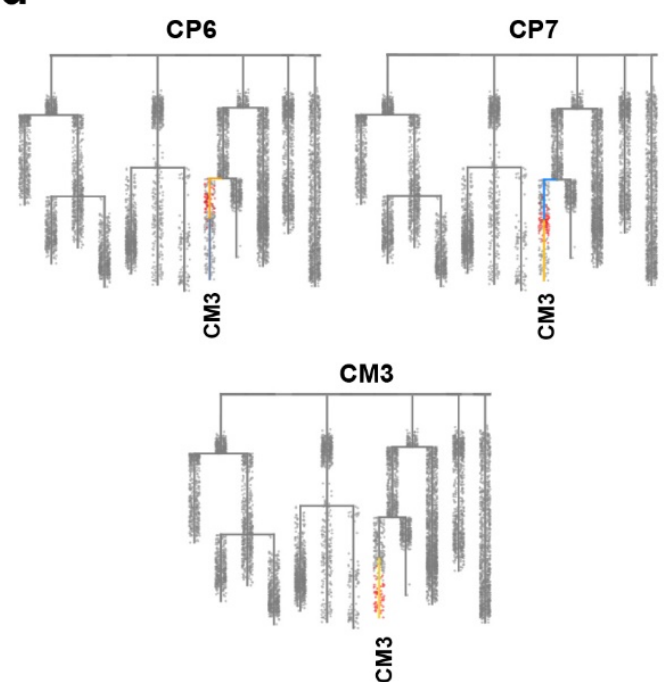
891 Extended Data Figure 5. Cardiac subcluster cell types are located in specific branches

892 of Mesp1-Cre URD developmental lineage tree. a-d, Each identified cardiac subcluster

893 from Figure $2 \mathbf{c}$ is projected onto the (a) URD developmental lineage tree from Figure $3 \mathbf{a}$.

894 Projections of these subclusters show that they reside in specific regions of the (b) CM1, (c)

895 CM2 and (d) CM3 branches. 


\section{Extended Data Figure 6}
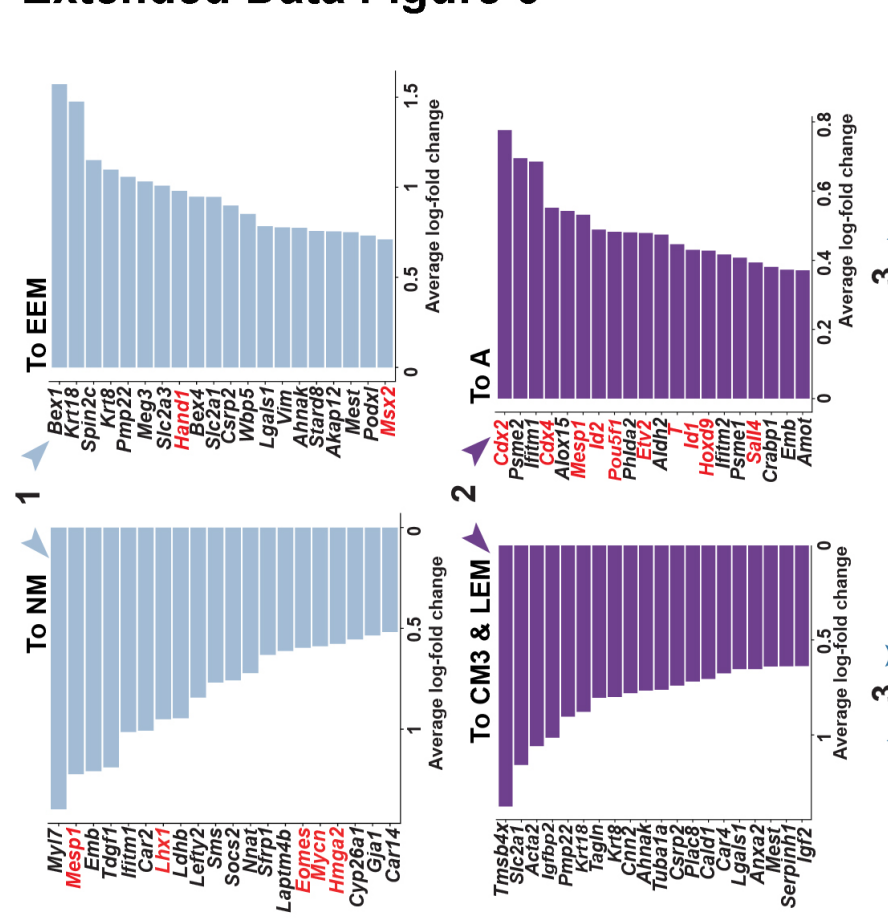

\section{$\frac{1}{2}$}
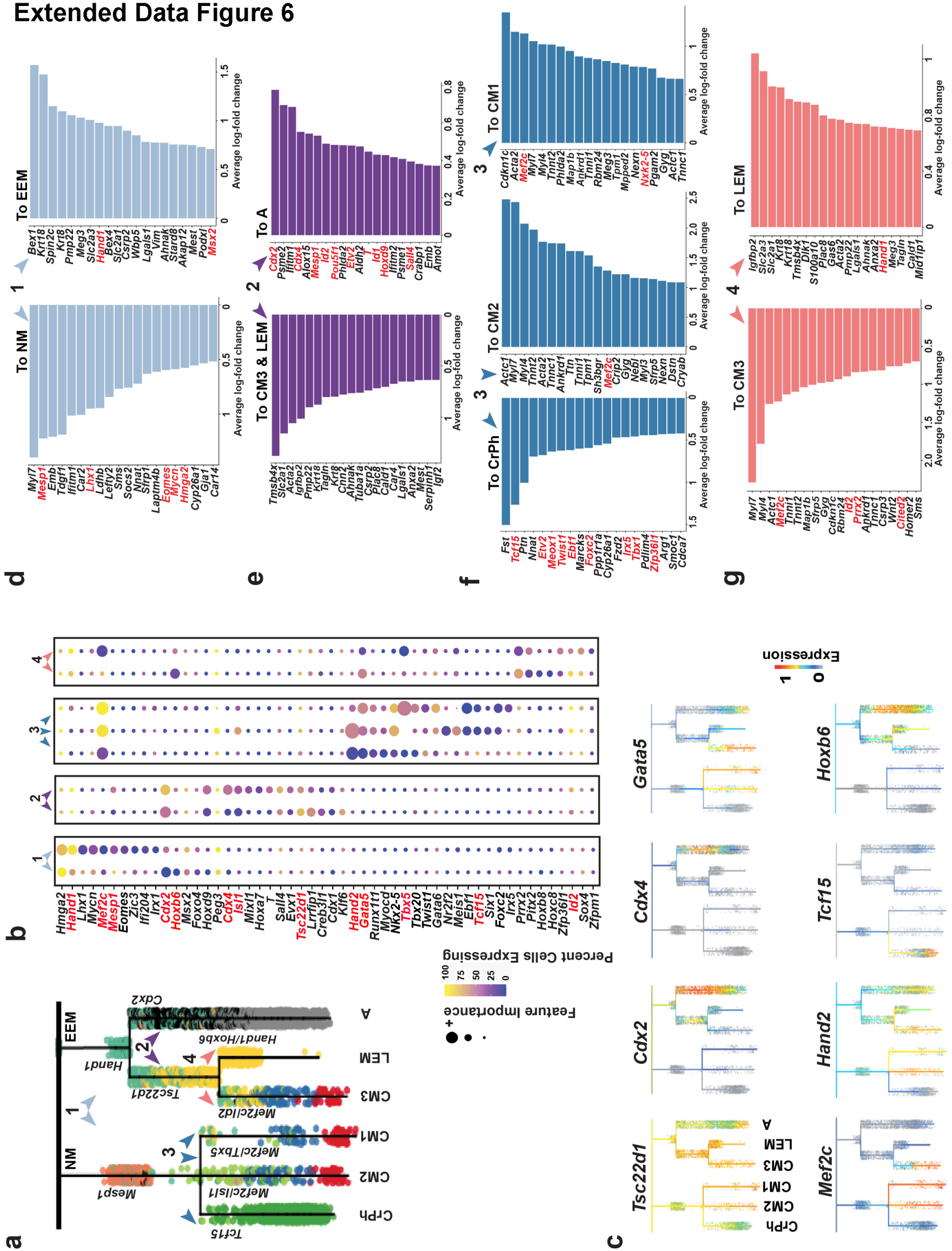

6u!̣sə.มdx $\exists$ s

\section{ำำ}

әэuеน. + .
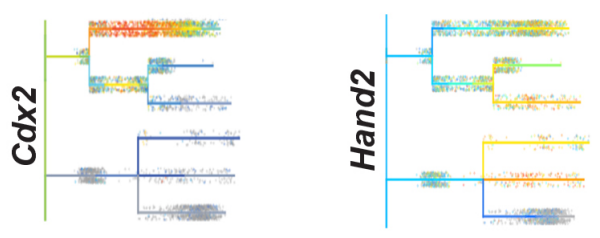

$\boldsymbol{U}$
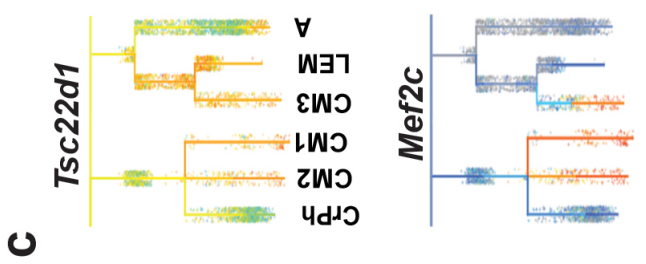
897 Extended Data Figure 6. Branch point analyses of Mesp1-Cre cardiac lineage

898 trajectories reveal molecular pathways for the development of distinct cardiac cell

899 types. a, The cardiomyocyte-related branches of the URD developmental tree display the

900 branch point decisions taken by cells differentiating into cardiomyocytes. Representative

901 important transcription factors as determined in $\mathbf{b}$ are indicated on the corresponding

902 branches of the URD developmental lineage tree. Cells are colored by their identity (see

903 Figure 1) and numbers indicate each designated branch point analyzed. Arrowheads point

904 to daughter branches. b, A random forest model was applied to predict the importance of

905 individual transcription factors in directing cells to specific daughter branches at branch

906 points labeled in a. The top ten transcription factors per branch, ranked by their importance,

907 are shown as noted by size of dots, which are colored by the percentage of cells in each

908 contrasting class/cells in the daughter branch just after the branch point. Red labeled genes

909 indicate representative transcription factors for each branch. c, The expression of

910 representative important transcription factors in $\mathbf{b}$ is projected onto the cardiomyocyte-related

911 branches of the URD developmental lineage tree (also see Figure 3c). d-g, Bar plots display

912 the most differentially expressed genes between the contrasting classes/cells in the daughter

913 branch just after each respective branch point (as indicated by numbers and arrowheads).

914 Transcription factors are labeled in red. Numbers and colored arrowheads indicate branch

915 points marked in panel (a).

916 


\section{Extended Data Figure 7}

\section{a}

CM1

pseudotime

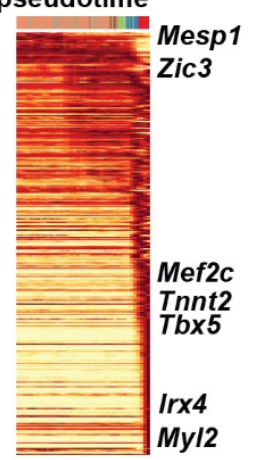

b

CM2

pseudotime

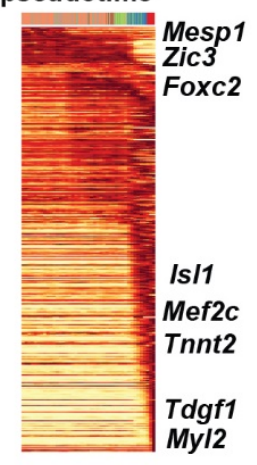

e

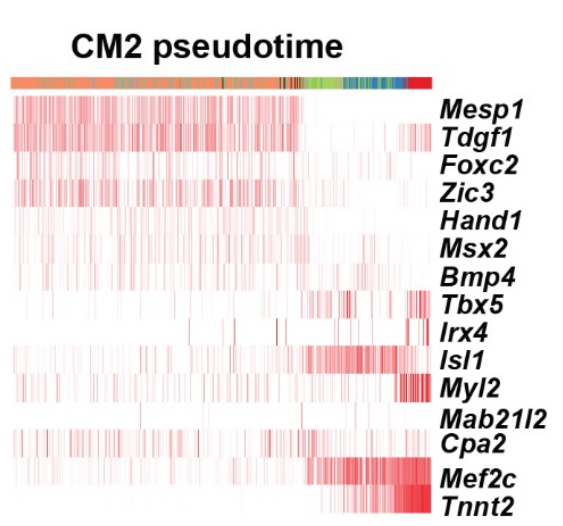

C

CM3
pseudotim

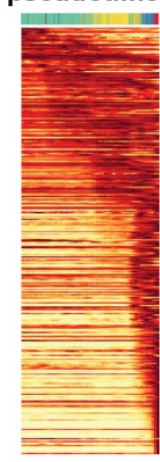

d

\section{CM1 pseudotime}

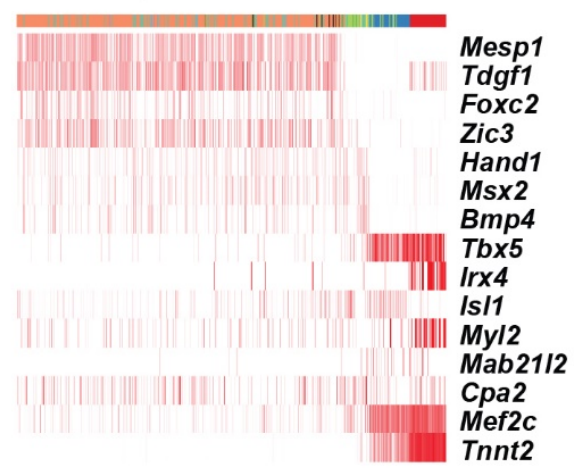

Tnnt2 g

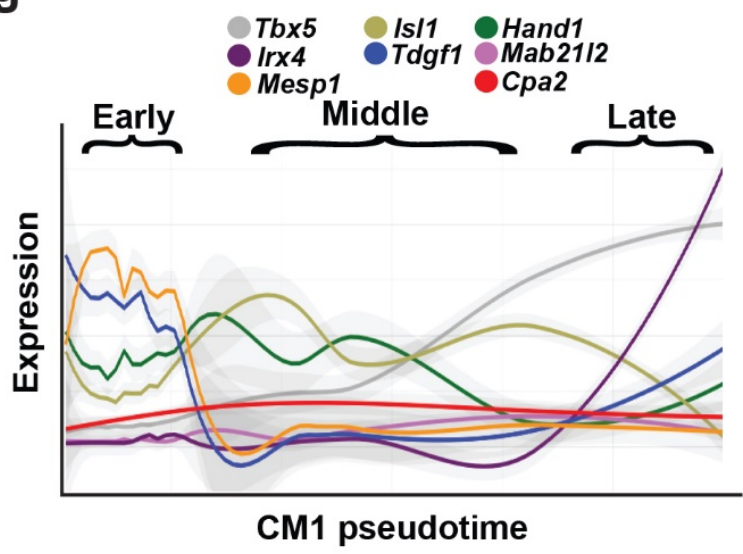

h

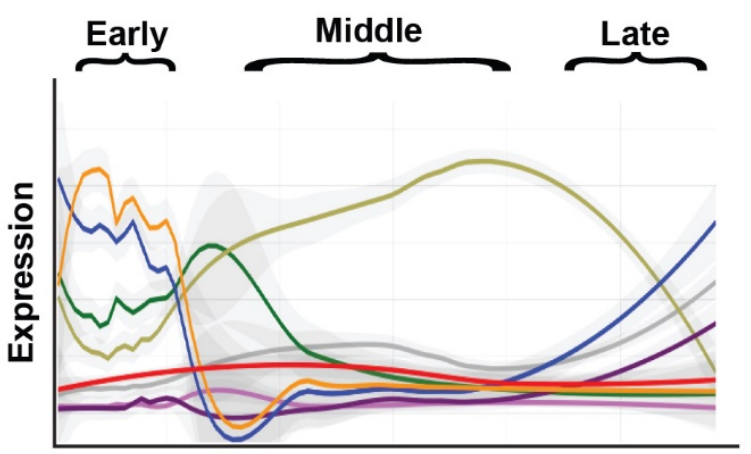

CM2 pseudotime

i

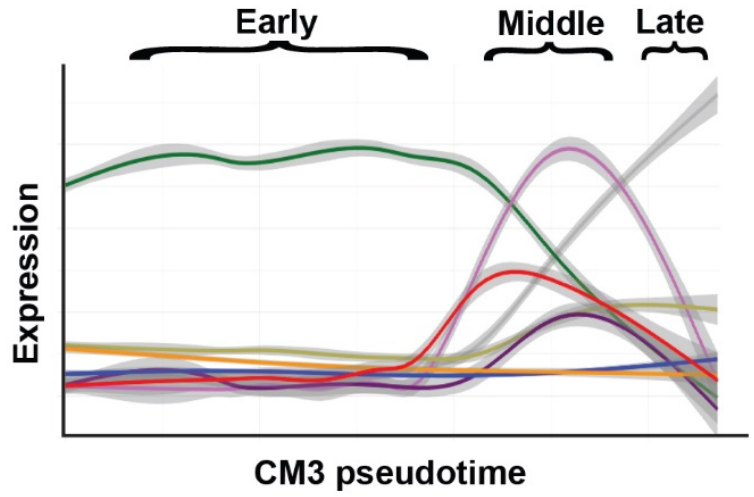




\section{Extended Data Figure 7. Pseudotime analyses of URD developmental lineage tree}

919 reveal the major developmental states and gene expression dynamics for each

920 cardiomyocyte subcluster cell type. a-c, Heatmaps of differentially expressed genes for

921 (a) CM1, (b) CM2 and (c) CM3 trajectories are displayed according to the pseudotime for

922 each respective trajectory. d-f, Heatmaps of selected genes for (d) CM1, (e) CM2 and (f)

923 CM3 trajectories are displayed according to the pseudotime for each respective trajectory.

924 Corresponding cell types that are ordered by pseudotime and colored according to their cell

925 identities in Figure 1 are shown at the top of each heatmap for a-f. g-i, Gene expression of

926 key markers for each cardiomyocyte subcluster trajectory is plotted along the pseudotime for

927 each of these trajectories $(\mathbf{g}-\mathrm{CM} 1, \mathbf{h}-\mathrm{CM} 2, \mathbf{i}-\mathrm{CM} 3)$. Colored lines indicate each gene

928 examined (see legend above plots). Brackets signify pseudotime stages for each

929 cardiomyocyte subcluster pseudotime plot. 


\section{Extended Data Figure 8}

Tbx5/Hand1/Is/1/DAP
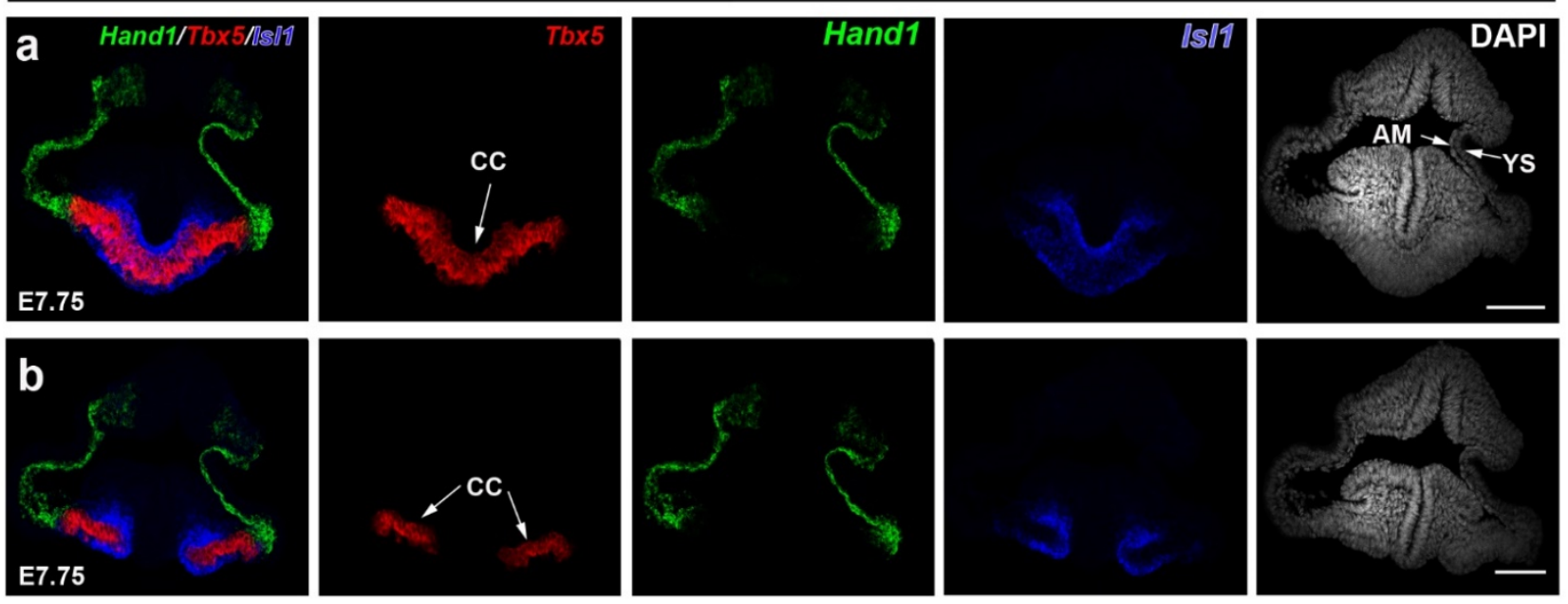
931 Extended Data Figure 8. Hand1, Tbx5 and Isl1 are expressed in specific domains of the

932 crescent region. a, b, RNAscope ISH studies on transverse sections of E7.75 embryos

933 reveal that Hand1, Tbx5 and Isl1 are expressed in distinct but complementary domains within

934 the crescent region. Furthermore, Handl is expressed in additional areas that are outside but

935 contiguous with the $T b x 5$-expressing cardiac crescent (CC). a, b panels show two different

936 levels of the crescent region. $\mathrm{n}=3$ embryos. Scale bars, $100 \mu \mathrm{m}$. AM, Amnion; YS, Yolk

937 Sac. 


\section{Extended Data Fig. 9}
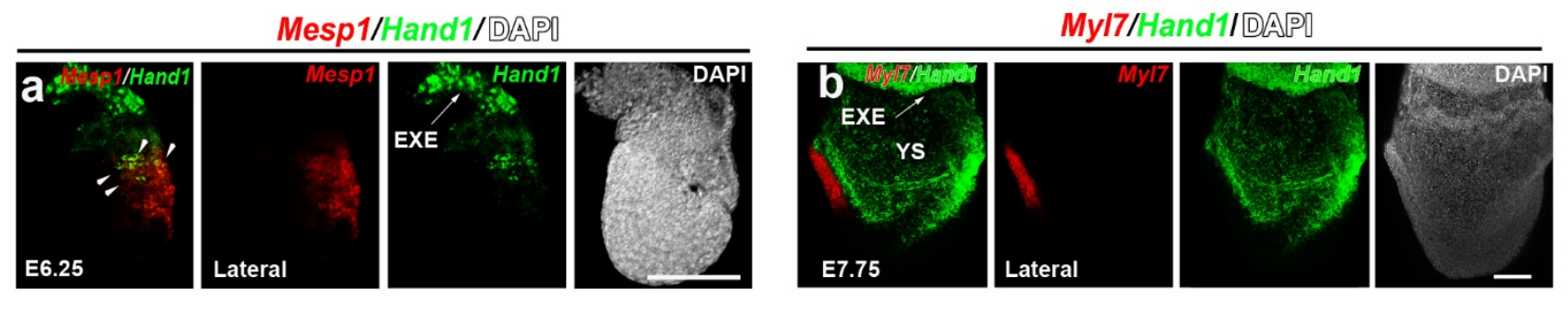

Hcn4/Hand1/Is/1/DAPP
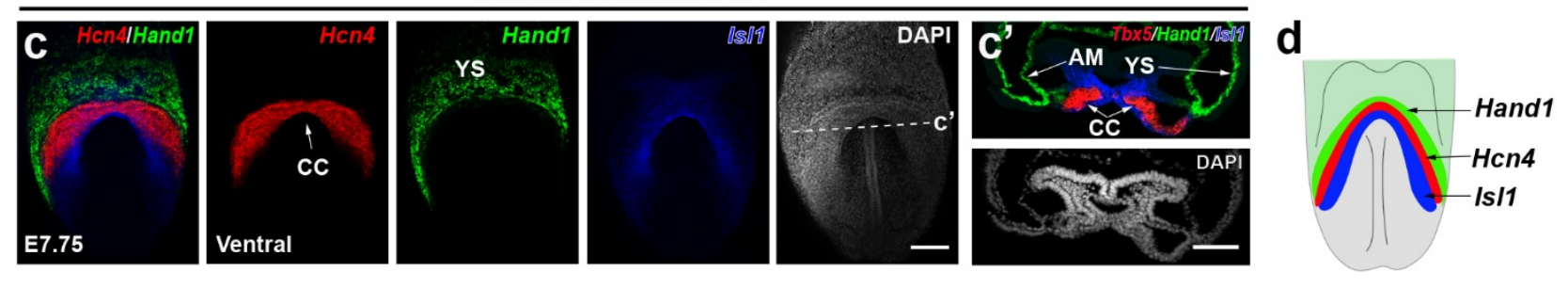

Myl7/Hand1/DAPD
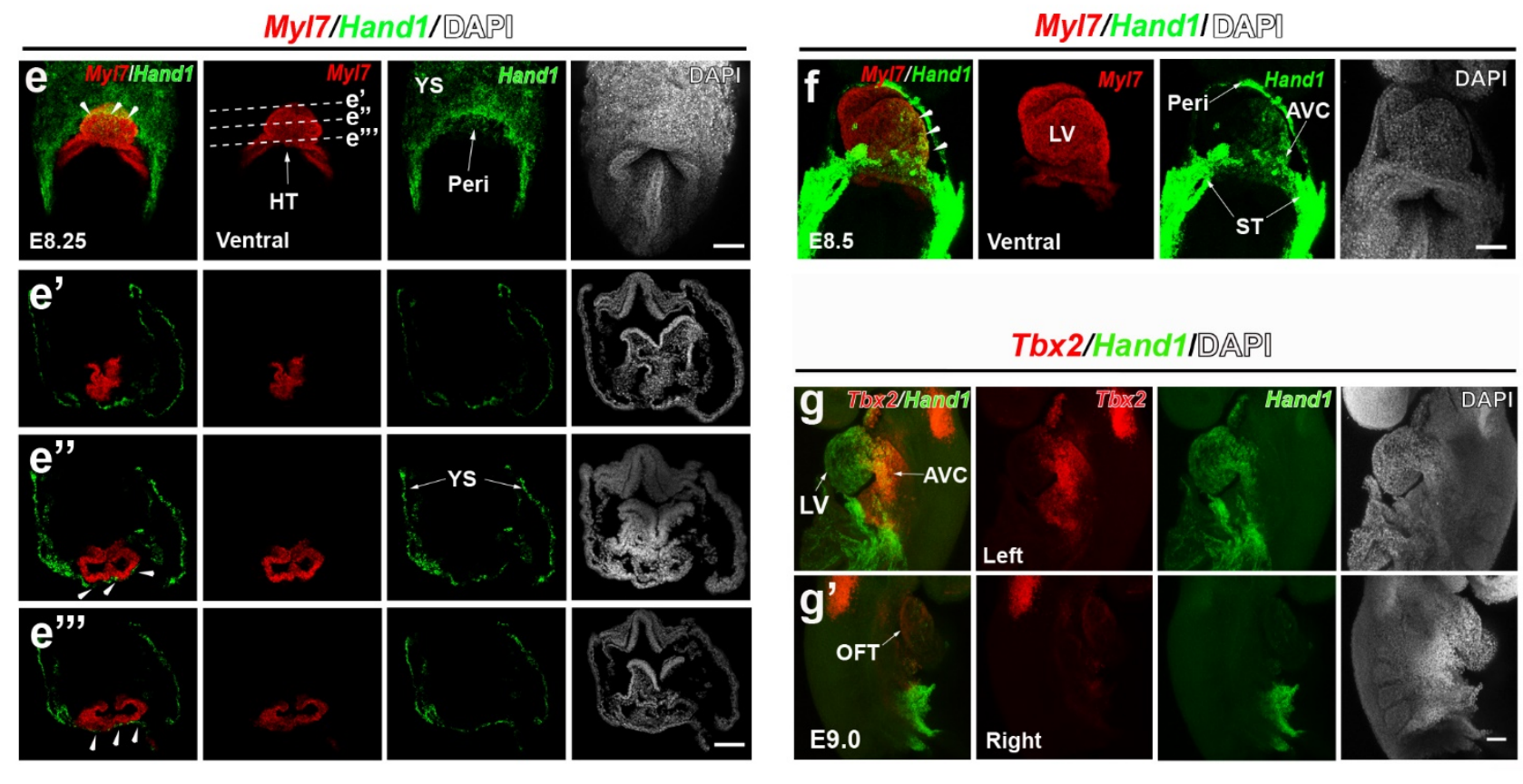
940 Extended Data Fig. 9. Hand1 exhibits spatiotemporally dynamic expression in

941 embryonic and extraembryonic tissues during embryogenesis. RNAscope in situ

942 hybridization (ISH) studies were performed across different stages of mouse development to

943 examine the dynamic expression of Hand1 during embryogenesis. a, Handl and Mespl are

944 co-expressed at the embryonic and extraembryonic boundary (arrowheads) in E6.25 embryos.

945 b, Handl is expressed in a region that is outside but contiguous with the Myl7 expressing

946 cardiac crescent in E7.75 embryos. c, d, Hand1 expression complements Hcn4 and Isl1

947 expression in the crescent region at E7.75. c', Inset shows transverse section of $\mathbf{c}$ at dashed

948 line. d, Diagram illustrates Hand1, Hcn4 and Isll expression in the crescent region as

949 shown in c. e, Handl is expressed in the yolk sac and pericardium (arrowheads) which

950 overlay the heart tube as detected by Myl7 expression, but is not expressed in differentiated

951 cardiomyocytes in E8.25 embryos. e', e', e',' Insets show transverse serial sections of the

952 heart tube at corresponding dashed lines in e. f, Hand1 is strongly expressed in the ST and

953 pericardium, but weakly expressed in $M y l 7+$ differentiated cardiomyocytes in the primitive

954 LV and AVC (arrowheads) in E8.5 embryos. g, g', Hand1 is expressed in the AVC (Tbx2+)

955 and LV, but is not expressed in the OFT in E9.0 embryos as shown in (g) left and ( $\left.\mathbf{g}^{\prime}\right)$ right

956 lateral views. $\mathrm{n}=3$ per panel. Scale bars, $100 \mu \mathrm{m}$. AM, Amnion; AVC, Atrioventricular

957 Canal; CC, Cardiac Crescent; EXE, Extraembryonic Ectoderm; Heart tube, HT; OFT,

958 Outflow Tract; Peri, Pericardium; ST, Septum Transversum; LV, Left Ventricle; YS, Yolk

959 Sac. 


\section{Extended Data Figure 10}
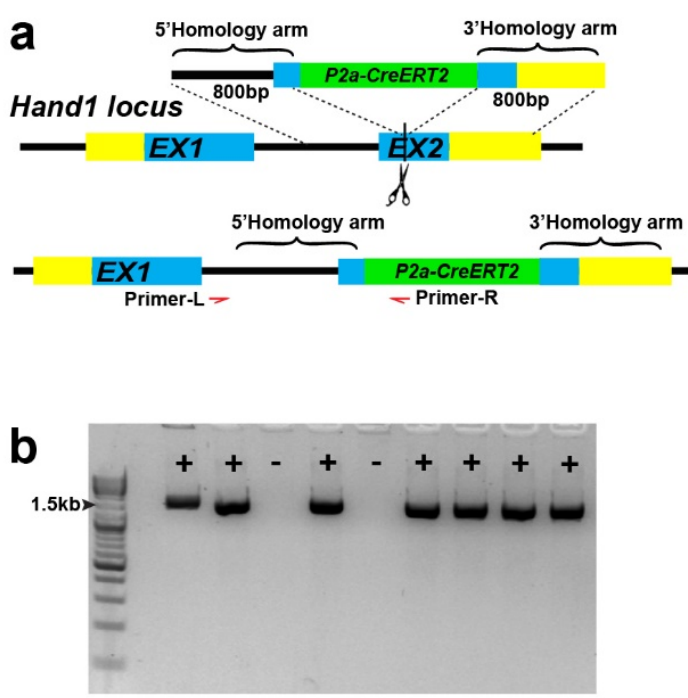

CreERT2+;Rosa26-tdT+

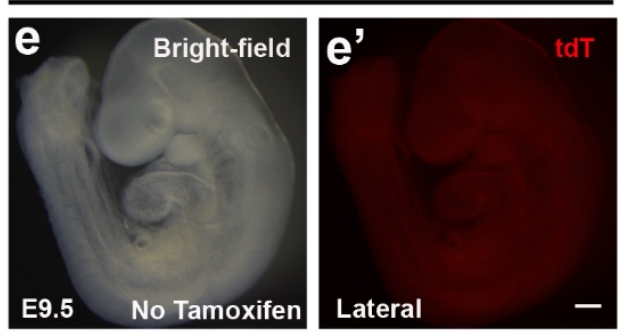

Hand1/CreERT2/DAPI

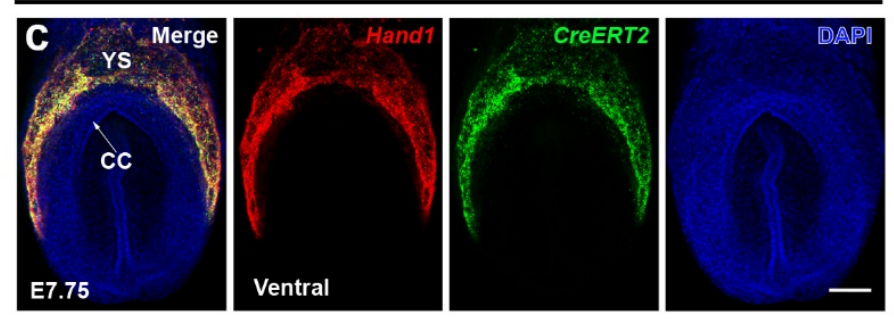

Hand1/CreERT2/DAPI

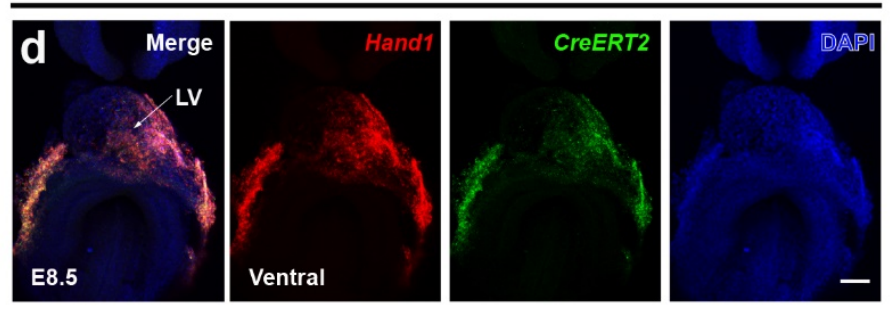


961 Extended Data Figure 10. Hand1-CreERT2 mouse line was generated by targeting a

962 P2a-CreERT2 construct into the second exon of the Hand1 locus. a, Schematic illustrates

963 the targeting strategy to create the Hand1-CreERT2 mouse line. b, PCR analysis shows the

964 genotyping for the identification of wildtype and Hand1-CreERT2 alleles. c, d, RNAscope

965 in situ hybridization studies reveal that the expression of CreERT2 from Hand1-CreERT2

966 mouse embryos recapitulates the endogenous expression of Hand1 at (c) E7.75 and (d) E8.5.

$967 \mathrm{n}=3$ per panel. Scale bars, $150 \mu \mathrm{m}$. e, In the absence of tamoxifen, no Hand1-CreERT2

968 genetically-labeled tdT+ cells were observed in the E9.5 Hand1-CreERT2; Rosa26-tdT

969 embryos. $\mathrm{n}=5$ embryos. Scale bars, $300 \mu \mathrm{m}$. CC, Cardiac Crescent; LV, Left Ventricle;

$970 \quad$ YS, Yolk Sac. 


\section{Extended Data Figure 11}

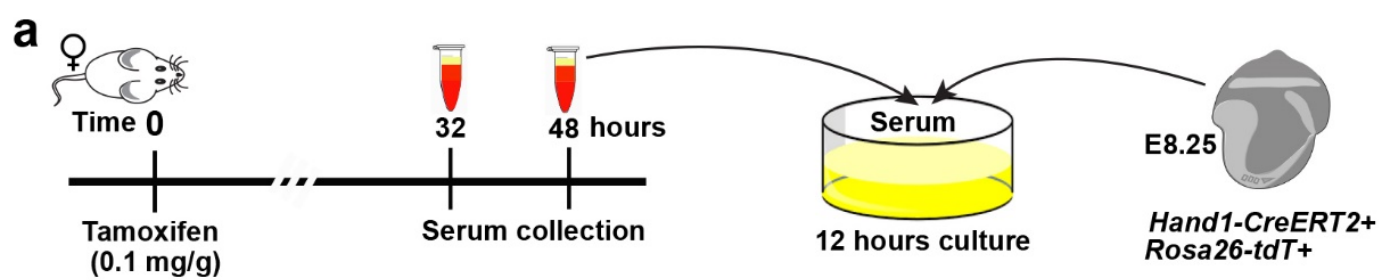

Hand1-CreERT2+; Rosa26-tdT+

Hand1-CreERT2+; Rosa26-tdT+
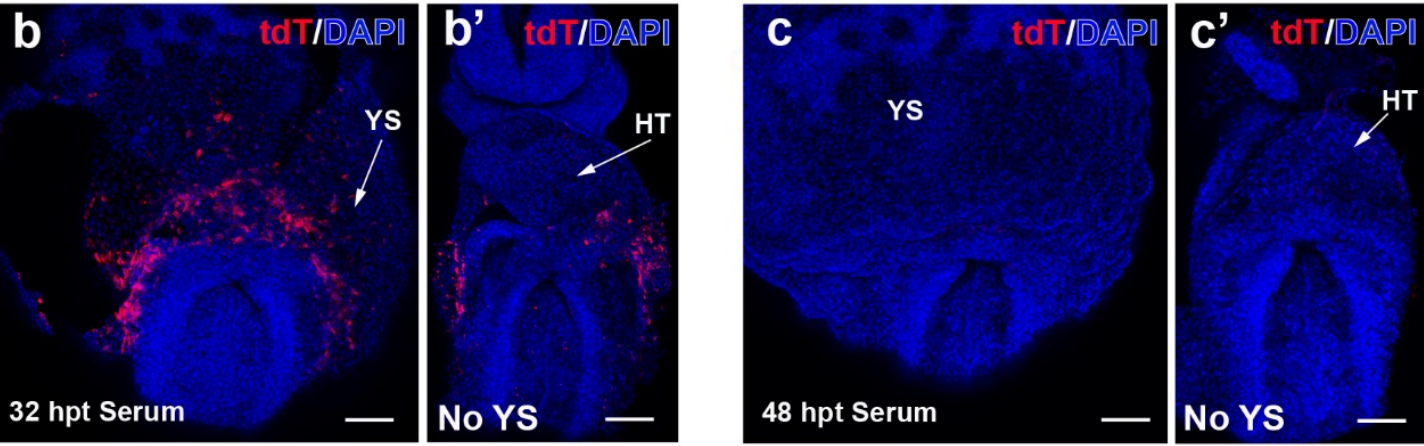
972 Extended Data Figure 11. Tamoxifen can induce recombination up to 32 hours after

973 treatment. a, Schematic illustrates experimental design for testing the perdurance of

974 tamoxifen after treatment. Wild-type Black Swiss adult females were given tamoxifen at a

$9750.1 \mathrm{mg} / \mathrm{g}$ dose, and serum was collected at 32 and 48 hours post tamoxifen treatment (hpt).

976 E8.25 Hand1-CreERT2; Rosa26-tdT embryos were cultured in collected sera for 12 hours.

977 b, b', These Hand1-CreERT2; Rosa26-tdT embryos cultured in serum collected 32 hours

978 after tamoxifen treatment exhibited some Hand1-CreERT2 genetically-labeled tdT + cells in

979 the yolk sac but not in the heart tube. $\mathrm{n}=3$. Scale bars, $150 \mu \mathrm{m} . \quad \mathbf{c}, \mathbf{c}^{\prime}$, However, no

980 genetically-labeled tdT+ cells were observed in Hand1-CreERT2; Rosa26-tdTomato embryos

981 cultured in serum collected 48 hours after tamoxifen treatment. $n=4$. Scale bars, $150 \mu m$.

982 The extraembryonic tissue and part of the pericardium tissue were removed in b', c' to show

983 the underlying heart tube. HT, Heart Tube; YS, Yolk Sac. 


\section{Extended Data Figure 12}

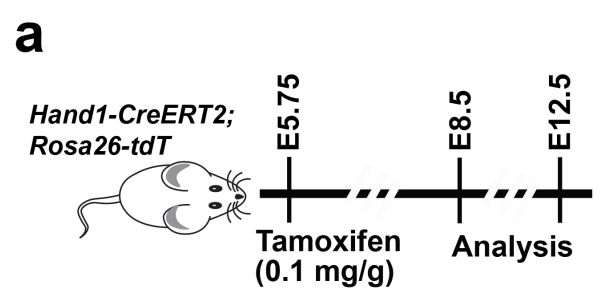

tdT/Wt1 /DAPI

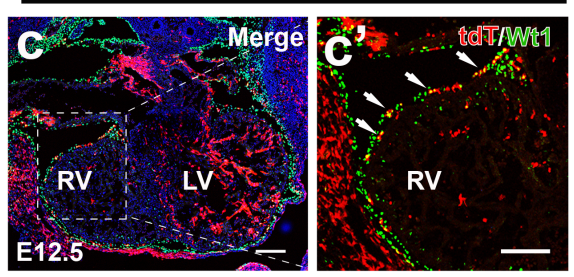

tdT/DAPI

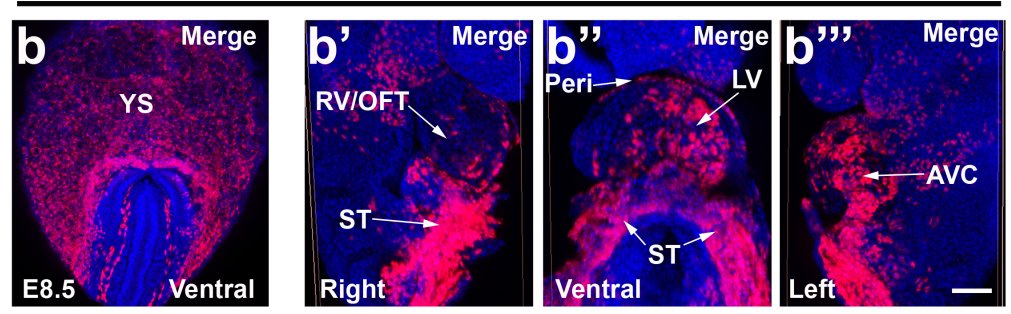

tdT/Erg1/Tnnt2

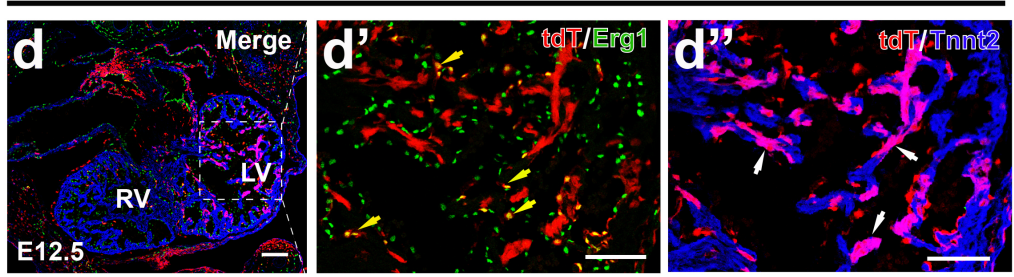

tdT/a-SMA/Pdgfr $\beta / \mathbb{D A} P[$
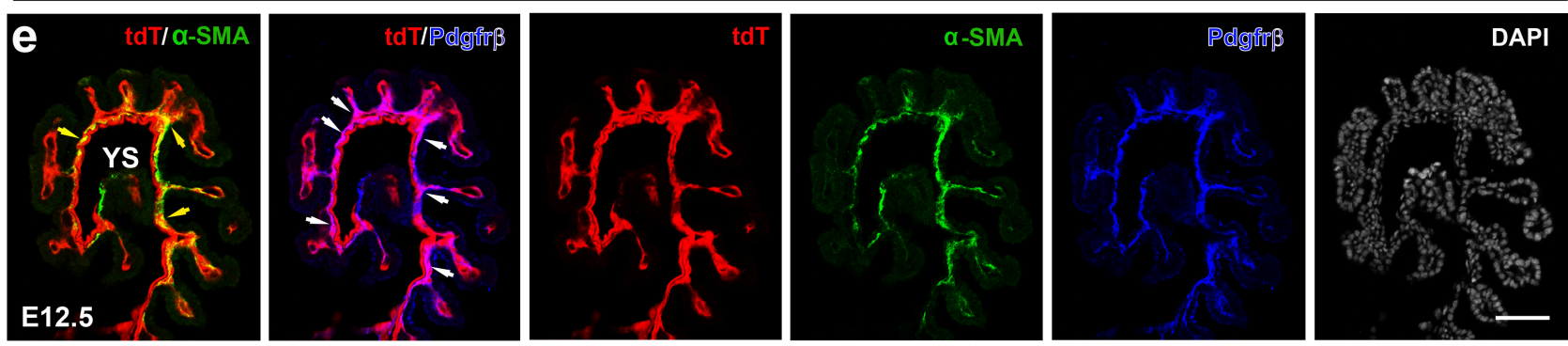

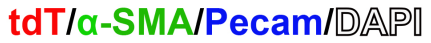
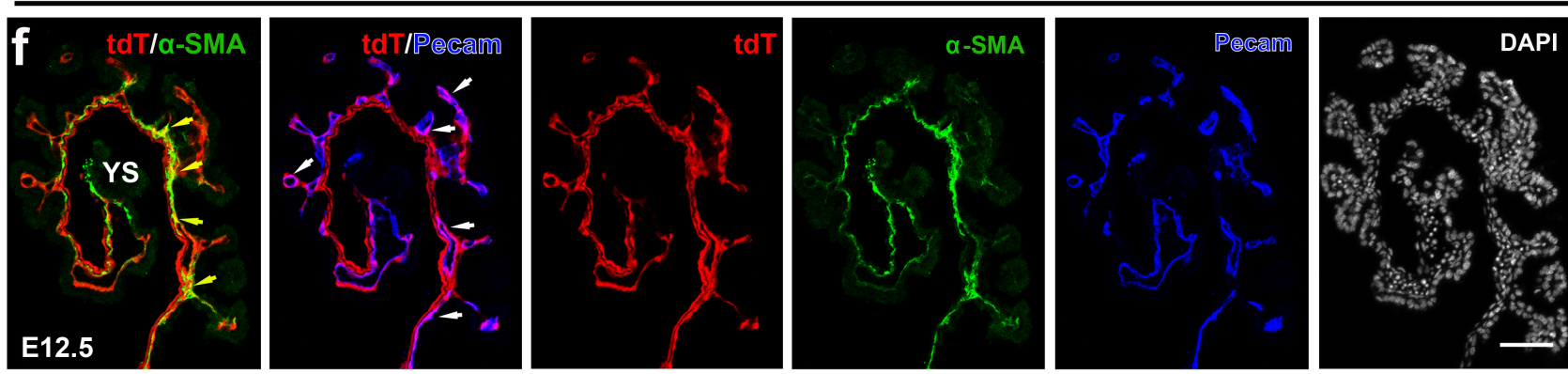
985 Extended Data Figure 12. Lineage tracing studies show that early gastrulating Hand1+

986

987

988

989

990

991

992

993

994

995

996

997

998

999

1000

1001

cells contribute specifically to left ventricular cardiomyocytes, epicardial and few

endocardial cells in the heart as well as endothelial cells, vascular support cells and

mesothelial cells in the yolk sac. a, Schematic illustrates the experimental strategy that was used to assess the contribution of E5.75 genetically-labeled Hand1-CreERT2; Rosa26-tdT

cells to E8.5 and E12.5 embryos. b, Whole mount embryo imaging shows that these Hand1-

CreERT2 genetically-labeled tdT + cells contribute to the yolk sac and developing heart at

E8.5. b', b', b',', The yolk sac (YS) and part of the pericardium tissue were removed in these panels to view the developing heart. c-f, Immunohistochemistry of cross-sectioned Hand1-CreERT2; Rosa26-tdT embryos at E12.5 reveals that Hand1-CreERT2 geneticallylabeled tdT + cells contribute to epicardial (c, c', Wt1, white arrowheads), myocardial (d, d', Tnnt2, white arrowheads) and few endocardial cells (d, d', Erg1, yellow arrowheads) in the heart as well as (e, f) smooth muscle cells ( $\alpha$-SMA, yellow arrowheads), (e) mesothelial cells (Pdgfr $\beta$, white arrowheads) and (f) endothelial cells (Pecam, white arrowheads) in the yolk sac. $\mathbf{c}^{\prime}, \mathbf{d} / \mathbf{d}$ ', Insets are magnification of $\mathbf{c}, \mathbf{d}$ boxed area, respectively. $\mathrm{n}=3$ per panel. Scale bars, $100 \mu \mathrm{m}$. AVC, Atrioventricular Canal; LV, Left Ventricle; OFT, Outflow Tract; Peri, Pericardium; ST, septum transversum; RV, Right Ventricle; YS, Yolk Sac. 


\section{Extended Data Figure 13}

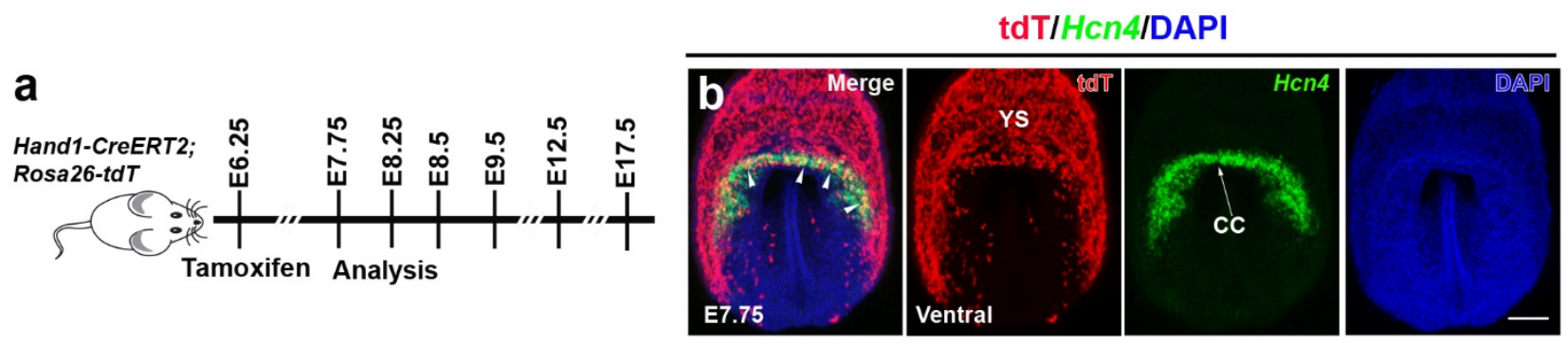

tdTIMyI7/DAPI
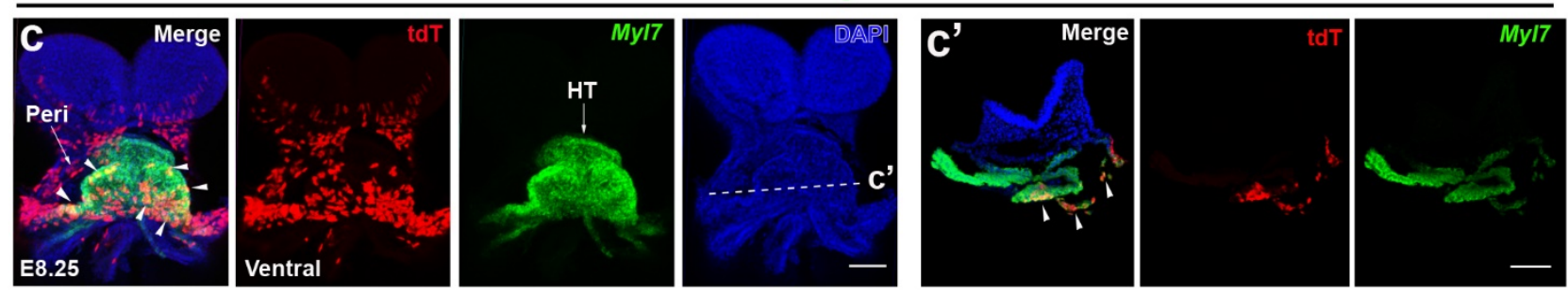

tdT/Myl7/DAPI
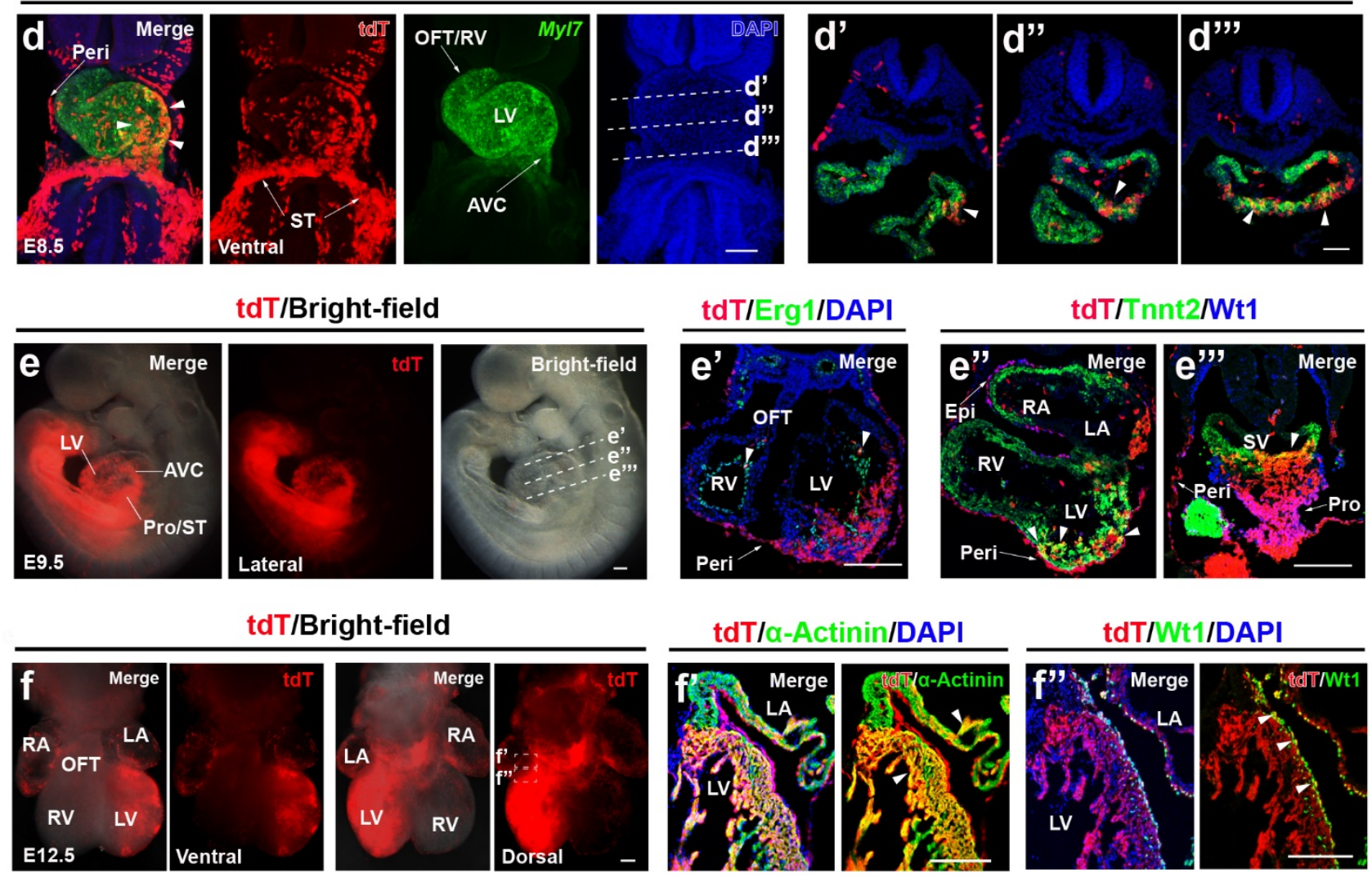

tdT/Bright-field
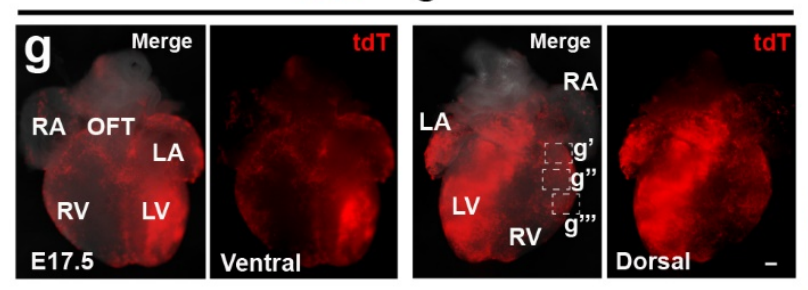

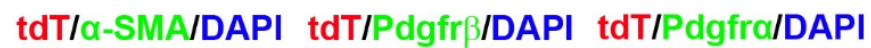
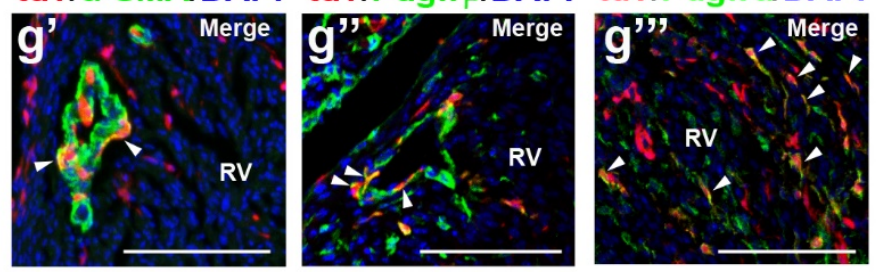
1003 Extended Data Figure 13. Lineage tracing studies marking Hand1+ progenitors at

1004 E6.25 reveal that these cells contribute to first heart lineage cardiomyocytes and serosal

1005 mesothelial lineages (pericardial, epicardial cells) in the heart. a, Schematic outlines the

1006 experimental strategy for Hand1-CreERT2 genetic fate mapping studies shown in b-g.

1007 Tamoxifen was given at E6.25, and Hand1-CreERT2; Rosa26-tdT embryos were examined

1008 for tdT localization at E7.75, E8.25, E8.5, E9.5, E12.5 and E17.5. RNAscope in situ

1009 hybridization and immunohistochemistry of whole mount and cross sections of these

1010 embryos (as indicated in each panel) reveal the contribution of Hand1-CreERT2 genetically-

1011 labeled tdT + cells at (b) E7.75, (c) E8.25, (d) E8.5 (e) E9.5, (f) E12.5 and (g) E17.5. c', d'-

1012 d"', e'-e", Insets show transverse sections of $\mathbf{c}, \mathbf{d}, \mathbf{e}$ at indicated dashed lines, respectively.

$1013 \mathbf{f}$ ', f'” and g'-g',', Inset shows representative coronal sections of $\mathbf{f}$ and $\mathbf{g}$ at indicated dashed

1014 boxes, respectively. Arrowheads point to tdT+ cells expressing (b) Hcn4, (c, d) Myl7, (e')

1015 Erg1, (e',) Tnnt2, (e',',f') Wt1, (f') $\alpha$-Actinin, (g') $\alpha$-SMA, (g',) Pdgfr $\beta$ and (g',') Pdgfr $\alpha$.

$1016 \mathrm{n}=3$ for each condition. Scale bars, $100 \mu \mathrm{m}$. Embryos analyzed at E17.5 were given 0.05

$1017 \mathrm{mg} / \mathrm{g}$ tamoxifen, half the dose given to embryos analyzed at earlier timepoints. AVC,

1018 Atrioventricular Canal; CC, Cardiac crescent; Epi, Epicardium; HT, Heart tube; LA, Left

1019 Atrium; LV, Left Ventricle; RA, Right Atrium; RV, Right Ventricle; OFT, Outflow Tract;

1020 Peri, Pericardium; Pro, Proepicardium; ST, Septum transversum; SV, Sinus Venosus; YS,

1021 Yolk sac. 


\section{Extended Data Figure 14}
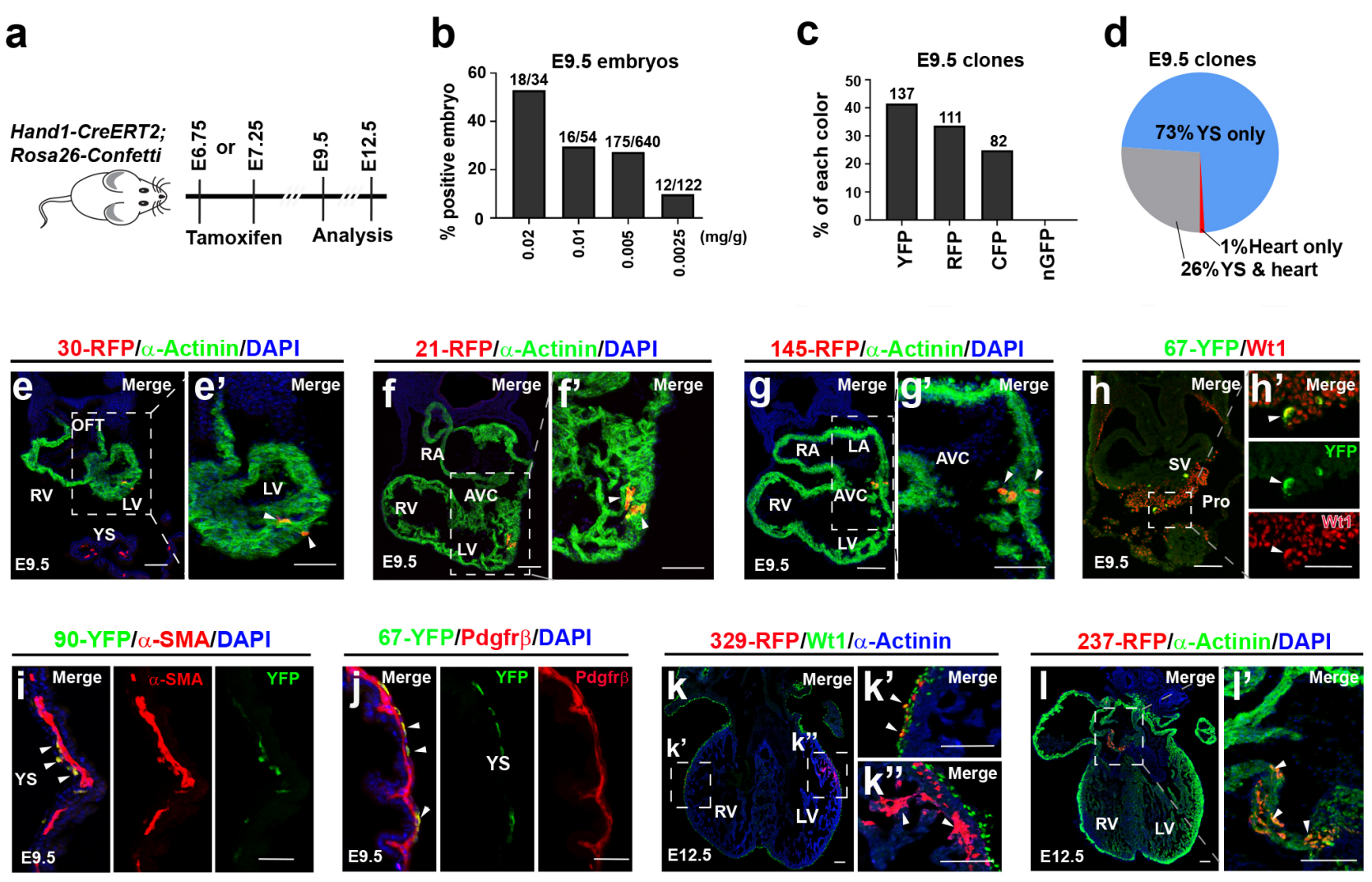

m

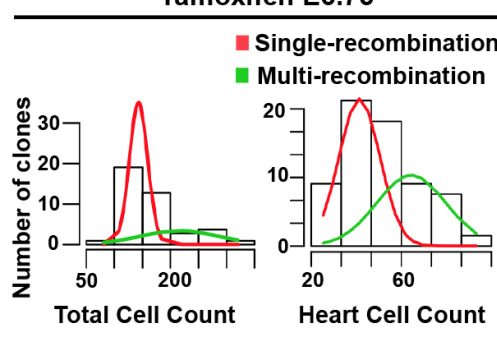

O

Tamoxifen E7.25

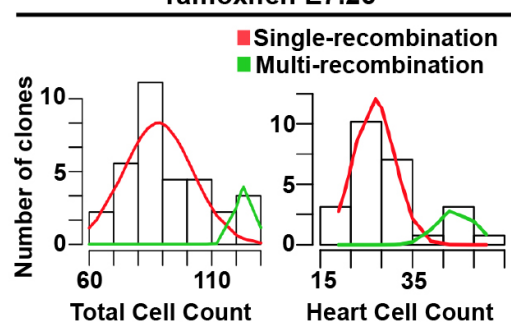

$\mathbf{n}$

Tamoxifen E6.75

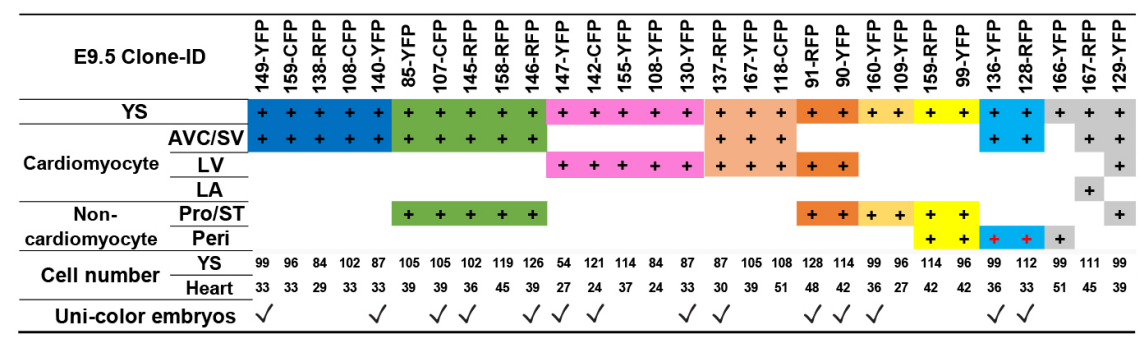

p

Tamoxifen E7.25

\begin{tabular}{|c|c|c|c|c|c|c|c|c|c|c|c|c|c|c|c|c|c|c|c|c|}
\hline \multicolumn{2}{|c|}{ E9.5 Clone-ID } & 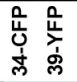 & 足 & 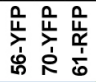 & 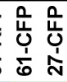 & 岁 & 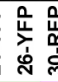 & 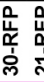 & 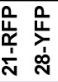 & $\begin{array}{l}\frac{0}{4} \\
\frac{\underline{r}}{i} \\
\dot{0}\end{array}$ & 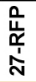 & $\begin{array}{l}\frac{0}{4} \\
\frac{1}{1} \\
\frac{1}{6}\end{array}$ & 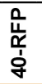 & & $\begin{array}{l}\frac{0}{4} \\
\frac{x}{1} \\
\text { op }\end{array}$ & 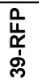 & 员 & 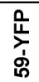 & \multirow{2}{*}{\multicolumn{2}{|c|}{ 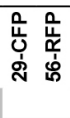 }} \\
\hline \multicolumn{2}{|l|}{ YS } & ++ & + & +++ & ++ & ++ & ++ & 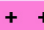 & ++ & + & + & + & + & + & + & + & + & + & & \\
\hline \multirow{2}{*}{ Cardiomyocyte } & AVC/SV & ++ & + & +++ & ++ & ++ & + & & & & & & & & & & & & & + \\
\hline & LV & & & & & & & 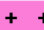 & ++ & & & & & & & & & + & & \\
\hline \multirow{2}{*}{$\begin{array}{c}\text { Non- } \\
\text { cardiomyocyte }\end{array}$} & Pro/ST & & & & ++ & ++ & + & & & + & + & + & + & + & + & + & & & + & \\
\hline & Peri & & & & & & & & & & & & & & & + & + & + & & \\
\hline \multirow{2}{*}{ Cell number } & YS & $56 \quad 63$ & 63 & $\begin{array}{lll}57 & 60 & 54\end{array}$ & 44256 & 660 & 814 & 447 & 7872 & 51 & 69 & 63 & 57 & 63 & 66 & 57 & 69 & 78 & 0 & 0 \\
\hline & Heart & $18 \quad 24$ & 21 & $\begin{array}{lll}24 & 24 & 21\end{array}$ & 1825 & $25 \quad 27$ & 272 & 212 & 2121 & 21 & 21 & 27 & 24 & 24 & 24 & 21 & 27 & 27 & 27 & 30 \\
\hline \multicolumn{2}{|c|}{ Uni-color embryos } & $\checkmark$ & & $\checkmark$ & & & $\checkmark v$ & $\checkmark v$ & $\checkmark$ & & & $\checkmark$ & & & & & & $\checkmark$ & $\checkmark$ & \\
\hline
\end{tabular}




\section{Extended Data Figure 14. Quantitative analysis of clones and their cell type} identification reveal the contribution of Hand1+ progenitor clones to specific cell

1026 lineages of the heart and yolk sac. a, Schematic outlines experimental strategy for Hand1-

1027 CreERT2; Rosa26-Confetti clonal analyses in b-p. b, Bar graph reveals the percentage of

1028 E9.5 embryos that displayed fluorescence at titrated doses of tamoxifen. c, Bar graph displays the frequency of each fluorophore expressed in E9.5 Hand1-CreERT2; Rosa26Confetti embryos that were induced with $0.005 \mathrm{mg} / \mathrm{g}$ tamoxifen. d, Pie chart shows the contribution of Hand1-CreERT2; Rosa26-Confetti clones to respective tissues. e-I,

1032 Immunohistochemistry of (e-j) E9.5 and (k-l) E12.5 Hand1-CreERT2; Rosa26-Confetti embryos reveals the contribution of Hand1-CreERT2 genetically-labeled clones to specific cell types including (e, f, $\mathbf{g}, \mathbf{k}, \mathbf{l})$ cardiomyocytes ( $\alpha$-Actinin) and $(\mathbf{h}, \mathbf{k})$ epicardial cells $(\mathrm{Wt} 1)$

1035 in the heart as well as (i) smooth muscle cells ( $\alpha$-SMA) and (j) mesothelial cells $(\operatorname{Pdgfr} \beta)$ in

1036 the yolk sac. $\mathbf{e}^{\prime}-\mathbf{h}^{\prime}, \mathbf{k}^{\prime}, \mathbf{k}, \mathbf{k}, \mathbf{l}$ ', Insets are magnification of $\mathbf{e}-\mathbf{h}, \mathbf{k}, \mathbf{l}$ boxed area. Arrowheads 1037 point to Hand1-CreERT2; Rosa26-Confetti labeled clones expressing (e', f', g', k', l') a1038 Actinin, (h', k') Wt1, (i) $\alpha-S M A$, and (j) Pdgfr $\beta$. ID number for each clone analyzed is 1039 indicated in panels. Scale bars $=100 \mu \mathrm{m} . \mathbf{m}, \mathbf{0}$, Histograms show the number of $(\mathbf{m})$ E6.75 1040 and (o) E7.25 genetically-labeled clones with a specific cell count (total or only in the heart).

1041 Gaussian distributions representing single (red line) and multiple (green line) recombinant 1042 events were modeled on this data. Based on these distributions, only clones that likely 1043 derived from a single recombination events were analyzed. These clones and their 1044 contributions to specific cell types are shown in (n) and (p), respectively. Unicolor embryos

1045 which are shown in Figure 5 are denoted by check mark on bottom of tables. AVC,

1046 Atrioventricular Canal; LA, Left Atrium; LV, Left Ventricle; RA, Right Atrium; RV, Right

1047 Ventricle; OFT, Outflow Tract; Peri, Pericardium; Pro, Proepicardium; ST, Septum

1048 transversum; SV, Sinus Venosus; YS, Yolk sac. 


\section{Supplementary Table 1. Differential gene expression analyses assign cell identities to}

scRNA-seq Mesp1-Cre clusters. Table displays differentially expressed marker genes for each cluster appearing in Figure 1 as defined in Methods. All tests are Wilcoxon rank sum tests. Column titles indicate the following information: gene - gene marker analyzed; cluster name - cluster expressing gene marker; $p \_v a l$ - unadjusted p-value of association with the cluster in contrast to all cells not in the cluster; avg_logFC average - average log2 change between the expression of the marker in the cluster versus all cells not in the cluster;

1057 pct. 1 - percentage of cells in the marked cluster that express the gene at a non-zero level;

1058 pct. 2 - percentage of cells not in the marked cluster that express the gene at a non-zero level; p_val_adj - multiple hypothesis adjusted p-value of association with the cluster in contrast to all cells not in the cluster; and cluster - numeric ID of the cluster as assigned in Figure 1.

\section{Supplementary Table 2. Differential gene expression analyses assign cell identities to}

1063 cardiac subclusters. Table displays differentially expressed genes for each cardiac

1064 subcluster appearing in Figure 2. All tests are Wilcoxon rank sum tests. Column titles

1065 indicate the following information: gene - gene marker analyzed; cluster name - cardiac

1066 subcluster expressing gene marker; p_val - unadjusted p-value of association with the

1067 subcluster in contrast to all cells not in the subcluster; avg_logFC average - average $\log 2$

1068 change between the expression of the marker in the subcluster versus all cells not in the

1069 subcluster; pct.1 - percentage of cells in the marked subcluster that express the gene at a non-

1070 zero level; pct. 2 - percentage of cells not in the marked subcluster that express the gene at a

1071 non-zero level; $p \_v a l \_a d j-$ multiple hypothesis adjusted p-value of association with the

1072 subcluster in contrast to all cells not in the subcluster. 
1074 Supplementary Table 3. Gene expression analyses reveals genes differentially

1075 expressed in branches at each branchpoint analyzed in the cardiomyocyte trajectories.

1076 Tables display genes that are differentially expressed between branches at each branch point

1077 analyzed in Extended Data Figure 6. All tests are Wilcoxon rank sum tests. Each sheet

1078 shows genes that are differentially expressed at corresponding branch points as indicated in

1079 the sheet name. Column titles indicate the following information: gene-gene marker

1080 analyzed; p_val - unadjusted p-value of association between two branches analyzed at

1081 respective branch point; avg_logFC average - average log2 change of gene expression

1082 between the two indicated branches analyzed; pct.1 and pct. 2 - percentage of cells

1083 expressing the gene in each branch as indicated in the column header; $p \_$val_adj - multiple

1084 hypothesis adjusted p-value of differential expression between the two branches.

1085

1086 Supplementary Table 4. Primer and sequence for making or genotyping Hand1-

1087 CreERT2, Rosa26-tdTomato and Rosa26-Confetti mice are shown. PCR primer,

1088 CreERT2 and gRNA sequences are provided for the making or genotyping of Hand1-

1089 CreERT2, Rosa26-tdT and Rosa26-Confetti mice. 


\section{Methods}

\section{Animal models}

1092 Animal studies were conducted in strict compliance with protocols approved by

1093 the Institutional Animal Care and Use Committee of the University of California, San Diego

1094 (UCSD) (A3033-01) and the Guide for the Care and Use of Laboratory Animals published

1095 by the National Institutes of Health. Mice were kept in IVC disposable cages (Innovive), under a 12-hour light cycle and bred on the Black Swiss background (Charles River Labs).

1097 We used Mesp1-Cre, Rosa26-tdT and Rosa26-Confetti mouse lines for our studies, which

1098 have been previously described ${ }^{19,20,63}$. The Hand1-CreERT2 knock-in line was made as

1099 described $^{76}$. Briefly, this procedure entailed using Gibson cloning to create a donor DNA

1100 fragment which contains a $P 2 a-C r e E R T 2$ sequence surrounded by 1600 bps of homology

1101 sequence to the second exon of Hand1 (See Supplementary Table 4 for primer sequences).

1102 This fragment was fully sequenced in order to ensure that mutations had not been introduced.

1103 The donor DNA $(0.6 \mu \mathrm{M})$ with Cas9 protein (NEB \#M0646T), crRNA and tracrRNA were

1104 injected in a 1:1:1:1 molar ratio into mouse zygotes by the UCSD Transgenic and Knockout

1105 Mouse Core (See Supplementary Table 4 for crRNA sequence). Four independent founders

1106 were recovered, of which three displayed strong Cre activity and expression. No differences

1107 were detected among these three founders, which were further propagated and used for

1108 experiments. Additionally, RNAscope in situ hybridization (ISH) confirmed that expression

1109 of CreERT2 from these mice recapitulates expression of endogenous Hand1 (Fig. 4e,

1110 Extended Data Fig. 10c, d). For genotyping, genomic DNA was extracted by adding $75 \mu 1$

1111 of $25 \mathrm{mM} \mathrm{NaOH}, 0.2 \mathrm{mM}$ EDTA to a $2 \mathrm{~mm}$ tail clipping and heating at $98^{\circ} \mathrm{C}$ for 30 minutes.

1112 The solution was then neutralized by adding $75 \mu \mathrm{l}$ of $40 \mathrm{mM}$ Tris- $\mathrm{HCl}(\mathrm{pH} 5.5)$. A 1:50

1113 dilution of genomic DNA template was used for genotyping PCR. Primers matching 
1114 sequences upstream of the left homology arm and in the Cre gene were used for genotyping

1115 (See Supplementary Table 4 for primer sequences.).

1116

\section{Embryo dissection and scRNA-seq library generation}

1118 To prepare single cells for scRNA-seq, Mesp1-Cre; Rosa26-tdT genetically-labeled embryos

1119 at E7.25, E7.5, E7.75 and E8.25 were dissected in cold sterile $1 \mathrm{X}$ PBS without $\mathrm{Ca}^{2+}, \mathrm{Mg}^{2+}$

1120 under a stereo microscope. Embryos were staged based on their morphology ${ }^{77}$. The

1121 Reichert's membrane and ectoplacental cone were removed, and Mesp1-Cre; Rosa26-tdT

1122 genetically-labeled embryos were selected and imaged. The yolk sac was removed from two

1123 of the three E8.25 embryos that were processed in order to enrich for cardiac cells.

1124 Individual embryos were placed into a $1.5 \mathrm{ml}$ microfuge tube and incubated in $0.25 \%$

1125 Trypsin-EDTA (Gibco, Catalog \# 25200056) at $37^{\circ} \mathrm{C}$ with inversion every two minutes for

112630 min until no visible tissue remained. The solution was pipetted once with a p1000 and

1127 neutralized by adding $0.75 \mathrm{ml}$ DMEM containing 10\% FBS (Gibco). Cells were then passed

1128 through a 100- $\mu$ m cell strainer (BD Biosciences, Catalog \# 352360) and single tdT + cells

1129 were obtained by fluorescence-activated cell sorting (FACS) on a BD Influx Cell sorter (BD

1130 Biosciences). Living cells were gated on FSC, SSC, DAPI- and tdT+. After sorting, cells

1131 were centrifuged at $300 \mathrm{~g}$ for 4 minutes and pooled or kept as individual embryos. Libraries

1132 were prepared using the Chromium Single Cell 3' Library and Gel Bead Kit v2 (PN-120237)

1133 and Chromium i7 Multiplex Kit (PN-120262) according to instructions from 10X Genomics

1134 (https://www.10xgenomics.com/resources/user-guides/). Prior to sequencing cDNA,

1135 libraries were verified by the D1000 ScreenTape system (Agilent) and quantified via Qubit ${ }^{\mathrm{TM}}$

1136 Flex Fluorometer (Thermofisher, Catalog \# Q33327). All libraries were sequenced twice on

1137 the HiSeq 4000 (Illumina) at the UCSD genomics core. An initial shallow sequencing run

1138 was done for quality control and to determine the number of cells captured. An individual 
1139 sample was excluded from further analysis due to a low number of reads per cell $(60 \%)$ as

1140 analyzed by Cell Ranger (10X genomics). A second deeper sequencing run was

1141 subsequently performed ensuring an approximate equal read depth per cell across the

1142 samples, resulting in an average of 60,450 UMIs (unique molecular identifiers) per cell and

1143 an average sequence saturation of $65.7 \%$ (Extended Data Fig. 1b).

1145 Data processing and clustering

1146 Reads were analyzed with the Seurat library (version 3.1.5). The data was read into the R

1147 (version 3.5.3) computing environment and log normalized using the Seurat library's

1148 NormalizeData() function with default parameters. Cells with more than 5 percent

1149 mitochondrial gene reads or less than 25,000 UMI were excluded. This analysis excluded

1150 approximately 1,400 cells (Extended Data Fig 1a, b). We then calculated the principal

1151 components of the data and used the first 10 principal components to calculate tSNE

1152 projections. Individual samples visualized in these tSNE projections revealed that samples

1153 overlapped, thus indicating a lack of batch effect (Extended Data Fig. 1c). In order to

1154 discover an optimal number of clusters for analysis, we also used ten principle components

1155 and calculated $\mathrm{k}$-means clustering for $\mathrm{k}=8$ to 25 . For each clustering solution $\mathrm{k}$, we

1156 observed the average silhouette score. We observed local maxima at $\mathrm{k}=10,12$ and 15 , and

1157 chose $\mathrm{k}=15$ for subsequent analysis (Extended Data Fig. 1d). For each of these clusters, we

1158 identified genes that were expressed at higher levels in that cluster compared to all other cells

1159 using default parameters for Seurat's FindMarkers function. The complete list of these

1160 genes along with the clusters that they represent can be found in Supplementary Table 1.

1161 These genes were examined more closely in order to assign a cell identity to each cluster.

1162 The most informative markers appear in Figure 1f. 


\section{Lineage inference}

1165 In order to infer the developmental relationships between cells in our study, we employed the

1166 R package URD (version 1.1.0) ) $^{28}$, which requires the user to declare certain cells to be part of

1167 the root or the tips of the cell lineage tree. URD then traces routes through a cell-cell nearest

1168 neighbor graph from the tip cells back to the root cells producing a tree-topology that

1169 summarizes the consensus routes from each tip back to the root cells. We used all cells from

1170 our earliest stage E7.25 (No bud) as the root of the tree, and cells of clusters that contained

1171 the most differentiated cell-types at the latest stage E8.25 (1-4 somite) as the tips. For the

1172 URD in Figure 2a, cells from E8.25 embryos from the following clusters were defined as

1173 tips: Allantois, A; Blood, B; Cardiomyocyte, CM; Cranial pharyngeal mesoderm, CrPh;

1174 Endothelium, E; Epithelium, EP; Lateral plate mesoderm, LPM; Late extraembryonic

1175 mesoderm, LEM; Pre-somitic mesoderm, PSM and Somite mesoderm, SM. For the URD in

1176 Figure 3a, the same clusters were defined as tips except the CM tip was split into three tips,

1177 based on the sub-clusters (CM1, CM2, CM3) defined by re-clustering only the cardiomyocyte

1178 branch as described in the results.

1179 To compare the Pijuan-Sala et al. data ${ }^{27}$ with our own, we first limited the analysis of

1180 their data to the developmental stages that we analyzed (E7.25 - E8.5). Analogous clusters

1181 between our data and theirs were determined by identifying the clusters which had the

1182 greatest number of identical marker genes. Marker genes for clusters in Pijuan-Sala et al.

1183 were defined as genes significantly associated to each cluster (adjusted p-val $<0.05$ ) as

1184 reported on their data portal (https://marionilab.cruk.cam.ac.uk/MouseGastrulation2018/).

1185 Marker genes in our data were determined as described above in Data processing and

1186 clustering section. To identify the clusters with the greatest number of identical marker

1187 genes between our dataset and the Pijuan-Sala et al. dataset, the number of marker genes

1188 from each of our clusters that match each of their clusters was divided by the total number of 
marker genes for the relevant cluster in our dataset. This value represents the size-

1190 normalized overlap between clusters in the two datasets and was plotted as a heatmap

1191 (Extended Data Fig. 3c). Clusters with maximal overlap were considered analogous

1192 between the two datasets. An URD tree was then created using analogous root (all E7.25

1193 cells) and tip clusters as defined above. The cardiomyocyte branch of this URD tree was

1194 shown (Extended Data Fig. 3d).

\section{Branch point differential analysis}

1197 At each branch point along the three cardiomyocyte developmental trajectories (Figure 3a,

1198 CM1, CM2, CM3), we used a Random Forest model ${ }^{44}$ to identify transcription factors likely

1199 to be responsible for cells choosing one branch over another. For this algorithm, we defined

1200 contrasting classes of cells as the first $\sim 300$ daughter cells for each branch that was compared

1201 at corresponding branch points. The feature set was defined as transcription factors (as

1202 identified by the Gene Ontology, DNA Binding, GO:0003677 term and manual annotation ${ }^{78}$ ).

1203 We used these contrasting classes and the feature set in the R library randomForest's main

1204 function randomForest() using default parameters (except the importance=TRUE option was

1205 set to return the feature importance measures). The importance measure used here is the

1206 mean decrease in accuracy measure (the default for RandomForest()), which quantifies the

1207 decrease in prediction accuracy of a class when the variable in question is randomly

1208 permuted. The top ten most important transcription factors that determined each class were

1209 plotted (Extended Data Fig. 6b). The differential analysis between branches at each branch

1210 point used the contrasting classes of cells defined above, but examined all genes (instead of

1211 just transcription factors) using Seurat's FindMarkers() function, with default parameters.

1212 The top twenty differentially positive expressed genes for each class as determined by their

1213 log-fold change were plotted (Extended Data Fig. 6d-g). 


\section{Pseudotime Trace Analysis}

1216 We used URD-defined pseudotime ${ }^{28}$ for our pseudotime analysis: which is the average

1217 number of transitions over edges of the nearest neighbor graph required to reach each cell

1218 from the root. In order to produce the pseudotime traces, we ordered cells along each

1219 lineage according to the URD-inferred pseudotime using URD's geneCascadeProcess()

1220 function (Extended Data Fig. 7a-c). The scaled expression from Seurat of each marker gene

1221 in each cell was plotted as a heatmap (Extended Data Fig. 7d-f) and as a smoothed spline

1222 (Extended Data Fig. 7g-i). Pseudotime stages (early, middle, late) were defined based on

1223 gene expression peak coherence in the smooth spline plots.

\section{Tamoxifen treatment}

1226 To determine the developmental stage of embryonic development during which tamoxifen

1227 treatment was administered, noon on the day of the vaginal plug was assumed to be E0.5.

1228 For lineage tracing studies, tamoxifen (Sigma, T5648-1G, $0.1 \mathrm{mg} / \mathrm{g}$ body weight) was fed to 1229 pregnant mice by gavage, except for embryos harvested at E17.5 when a lower dose of

1230 tamoxifen was used $(0.05 \mathrm{mg} / \mathrm{g}$ body weight $)$. For the clonal analysis, tamoxifen was

1231 administered by intraperitoneal injection.

1232

1233 Lineage tracing and clonal analysis

1234 For lineage and clonal analyses, Hand1-CreERT2 mice were crossed with Rosa26-tdT or

1235 Rosa26-Confetti mice respectively. Genetically-labeled embryos were identified using a

1236 fluorescent stereo microscope (ZEISS AXIO Zoom.V16 or LEICA M205 FA). Embryos

1237 younger than E9.5 were imaged using a confocal microscope (Nikon C2), while embryos

1238 older than E9.5 were imaged with a fluorescent stereo microscope and then imaged with the 
confocal microscope after sectioning. Embryos were embedded and sectioned into 10 or 20

$1240 \mu \mathrm{m}$ sections for lineage tracing or clonal analysis, respectively. To determine the number of

1241 cells in a clone, sections from an individual embryo were processed and distributed evenly

1242 across three slides for E9.5 embryos, or five slides for E12.5 hearts. All cells from an

1243 individual clone on one slide were then counted. The total number of cells per clone was

1244 then calculated by multiplying the number of cells in a clone on a single slide by the number

1245 of slides.

1246 Tamoxifen perdurance was determined by incubating E8.25 embryos in serum collected

1247 from Black Swiss females 32 or 48 hours after they were given tamoxifen $(0.1 \mathrm{mg} / \mathrm{g})$.

1248 Serum was collected by centrifuging ( $2 \mathrm{x} 400 \mathrm{~g}$ for $6 \mathrm{mins})$ blood collected via retro-orbital

1249 bleeding and then frozen at $-80^{\circ} \mathrm{C}$. Separately, E8.25 embryos were obtained from Hand1-

1250 CreERT2 x Rosa26-tdT crosses without tamoxifen administration and dissected in 5\%

1251 FBS/Fluorobrite DMEM media (ThermoFisher, Cat. no. A1896701 and 10082139 ) on a $37^{\circ} \mathrm{C}$

1252 heated stage (Tokai Hit, TPi-SZX2AX). Care was taken to remove the Reichert's

1253 membrane, but not the ectoplacental cone. Embryos were then incubated at $37^{\circ} \mathrm{C}$ in $5 \% \mathrm{CO}_{2}$

1254 for 12 hours in $2 \mathrm{ml}$ of pre-warmed serum collected from tamoxifen-injected females. After

1255 incubation, embryos were fixed in 4\% PFA and processed for immunofluorescence with anti-

1256 tdT antibody. Embryos which did not display tdT were genotyped to confirm that they

1257 contained both Hand1-CreERT2 and Rosa26-tdT DNA. Three independent experiments

1258 were performed.

\section{RNAscope Fluorescent in situ hybridization}

1261 Whole-mount RNAscope fluorescent in situ hybridizations (ISH) were conducted using the

1262 RNA-scope Multiplex Fluorescent Reagent Kit v.2 (Advanced Cell Diagnostics, 323100)

1263 with several adaptations. Embryos were dissected in RNase-free 1X PBS and fixed in 4\% 
1264 PFA overnight at $4^{\circ} \mathrm{C}$. Embryos were then washed 3 times in $0.1 \%$ Tween20/PBS (PBT),

1265 followed by dehydration into and then re-hydration from methanol using 5 minute $25 \%, 50 \%$,

$126675 \%$ and $100 \%$ Methanol/PBT washes. Probe hybridization was performed at $50^{\circ} \mathrm{C}$. For

1267 samples that were co-stained with antibodies after the RNAscope ISH, samples were

1268 incubated in $10 \%$ heat-inactivated donkey serum for 2 hours at room temperature prior to

1269 addition of primary antibody (see below) overnight at $4^{\circ} \mathrm{C}$. The embryos were then washed

$12703 x$ in PBT and incubated in secondary antibody (see below) for 2 hours in $4{ }^{\circ} \mathrm{C}$. Whole-

1271 mount embryos were imaged after mounting in 1\% low melting point agarose in $35 \mathrm{~mm}$ glass

1272 bottom petri dishes (MatTek) using a confocal microscope (Nikon C2). After imaging,

1273 embryos were embedded and sectioned for further analysis as described in the

1274 Immunofluorescence, sectioning and image processing section. Catalog numbers for RNA-

1275 scope probes (ACDbio) used in this study: Cre-O4-C1, Cat No. 546951; Mm-Hcn4-C1, Cat

1276 No. 421271; Mm-Hand1-C1, Cat No. 429651; Mm-Hand1-C2, Cat No. 429651-C2; Mm-

1277 Is11-C2, Cat No. 451931-C2; Mm-Mab2112-C1, Cat No. 456901; Mm-My17-C3, Cat No.

1278 584271-C3; Mm-Mesp1-C3, Cat No. 436281-C3; Mm-Nkx2-5, Cat No. 428241; Mm-Nkx2-

1279 5-C2, Cat No. 428241-C2; Mm-Nr2f2, Cat No. 480301; Mm-Sfrp5-C1, Cat No. 405001;

1280 Mm-Smoc2-C1, Cat No. 318541; Mm-Tbx5-C1, Cat No. 519581; Mm-Irx4-C1, Cat No.

1281 504831; Mm-Tbx5-C2, Cat No. 519581-C2; Mm-Tdgf1-C1, Cat No. 506411.

1282

1283 Immunofluorescence, sectioning and image processing

1284 Immunofluorescence studies were conducted on cryosections of mouse embryos. Embryos

1285 were cryoprotected, mounted, sectioned and stained as we previously described ${ }^{12}$. The

1286 following primary antibodies were used: mouse anti-TNNT2 antibody (Invitrogen, Catalog \#

1287 MA5-12960, 1:50), Chicken anti-GFP antibody (Abcam, ab13970, 1:300), Rabbit anti-ERG1

1288 antibody (Abcam, ab92513, 1:300), Rabbit anti-WT1(Abcam, ab89901, 1:200), Rabbit anti- 
PDGFR $\alpha$ antibody (Abcam, ab203491, 1:200), Rabbit anti-PDGFR $\beta$ antibody (Abcam, ab32570, 1:200), Rat Anti-Mouse CD31 (BD Pharmingen, cat\# 553708, 1:500), Rabbit anti-

$1291 \alpha$-smooth-muscle-actin (Abcam, ab15734, 1:200), Rabbit anti- $\alpha$-Actinin (Abcam, ab68167,

1292 1:200), Mouse anti- $\alpha$-Actinin(Sigma, A-7811, 1:500), Goat Anti-tdTomato (SICGEN,

1293 AB8181-200, 1:500). The following secondary antibodies were diluted 1:250 in

1294 0.125\%PBST with DAPI (Invitrogen, Catalog \# D1306, 1:1000) and incubated for 1.5 hour at

1295 RT: Donkey Anti-Rabbit IgG-Alexa 488, 594, 647 (Invitrogen, Catalog \# A32790, \# A32754,

1296 \# A32795); Donkey anti-Goat IgG-Alexa 594 (Invitrogen, Catalog \# SA5-10088), Donkey

1297 anti-Mouse IgG-Alexa 488 (Invitrogen, Catalog \# A32766), Goat anti-Chicken IgG-Alexa

1298488 (Invitrogen, Catalog \# A32931), Donkey anti-Rat IgG-Alexa 488 (Invitrogen, Catalog

1299 \# A-21208). All images were processed using Nikon NIS Elements software, ImageJ and

1300 Adobe Illustrator.

1301

\section{Statistical Analysis of Clonal Events}

1303 To ensure that the clones we analyzed were the result of a single recombination event, we

1304 employed several different techniques including 1) using a low dose of tamoxifen, 2) utilizing

1305 the Rosa26-Confetti mouse line in which for each recombination event, only one of four

1306 different fluorophores is expressed, 3) separately analyzing unicolor embryos as well as

1307 multi-color embryos and 4) applying a rigorous statistical analysis based on the number of

1308 cells in a clone in order to exclude single color clones that may have resulted from two

1309 recombinant events. This statistical analysis involved embryos harvested at E9.5 in which

1310 single color clones were noted in both extraembryonic and cardiac regions. In these

1311 embryos, we calculated the number of cells in each clone. A mixture of two Gaussian

1312 distributions ${ }^{64}$ was fit to the data using expectation maximization. The distribution for the

1313 counts was then given by: 


$$
C=\alpha_{1} N\left(\mu_{1}, \sigma_{1}\right)+\alpha_{2} N\left(\mu_{2}, \sigma_{2}\right)
$$

1316 with:

$$
\mu_{1}<\mu_{2}
$$

$1318 \alpha_{1}, \alpha_{2}$ were the mixing parameters, and $N(\mu, \sigma)$ was a Gaussian distribution with mean $\mu$ and

1319 standard deviation $\sigma$. The first of the Gaussian distributions represented the cell count

1320 distribution from a single recombination event while the other represented the cell count

1321 distribution from multi-recombination events. This analysis was performed separately on

1322 E6.75 and E7.25 induced clones. Clones which had a likelihood of belonging to the

1323 Gaussian distribution with a smaller mean were consider clonal and the clones that fit the

1324 larger mean Gaussian distribution were considered multiclonal and were excluded from our

1325 analysis of multipotentiality.

1326

\section{Statistics and reproducibility}

1328 Replicates and statistical tests are described in the figure legends. No statistical methods

1329 were used to predetermine sample size. Experiments did not employ randomization nor

1330 investigator blinding. All experimental results were analyzed with at least three independent

1331 embryos. Wilcoxon rank sum tests were used for statistical tests for differential gene

1332 expression analysis including the tests supporting the box and whisker plots. Markers were

1333 defined by Seurat's default settings (at least $>0.25 \log$ fold increase over the opposing group, 1334 at most $<0.01$ unadjusted $p$-value, and at least $>10 \%$ cells expressing). On the boxplots, a p-

1335 value $<0.01$ was considered to be statistically significant as indicated by $*$. Box and

1336 whisker plots were created with standard parameters from ggplot2.

\section{Reporting summary}


1339 Further information on research design is available in the Nature Research Reporting

1340 Summary linked to this paper.

\section{Data and code availability}

1343 The scRNA-Seq data set supporting results of this article is available in the GEO database.

1344 Visualization of gene expression of the scRNA-seq is available on the UCSC cell browser at

1345 https://cells.ucsc.edu/. The R scripts are available upon request.

\section{Method References}

134812 Liang, X. et al. HCN4 dynamically marks the first heart field and conduction system precursors. Circulation research 113, 399-407, doi:10.1161/circresaha.113.301588 (2013).

19 Saga, Y. et al. MesP1 is expressed in the heart precursor cells and required for the formation of a single heart tube. Development (Cambridge, England) 126, 3437-3447 (1999). system for the whole mouse brain. Nature neuroscience 13, 133-140, doi:10.1038/nn.2467 (2010).

27 Pijuan-Sala, B. et al. A single-cell molecular map of mouse gastrulation and early organogenesis. Nature 566, 490-495, doi:10.1038/s41586-019-0933-9 (2019).

28 Farrell, J. A. et al. Single-cell reconstruction of developmental trajectories during zebrafish embryogenesis. Science (New York, N.Y.) 360, doi:10.1126/science.aar3131 (2018).

63 Snippert, H. J. et al. Intestinal crypt homeostasis results from neutral competition between symmetrically dividing Lgr5 stem cells. Cell 143, 134-144, doi:10.1016/j.cell.2010.09.016 (2010).

64 Benaglia, T., Chauveau, D., Hunter, D., R. \& Young, D., S. mixtools: An R Package for Analyzing Finite Mixture Models. Journal of Statistical Software 32, 1-29 (2009).

76 Yao, X. et al. Tild-CRISPR Allows for Efficient and Precise Gene Knockin in Mouse and Human Cells. Developmental cell 45, 526-536.e525, doi:10.1016/j.devcel.2018.04.021 (2018).

77 Downs, K. M. \& Davies, T. Staging of gastrulating mouse embryos by morphological landmarks in the dissecting microscope. Development (Cambridge, England) 118, 1255-1266 (1993).

78 Harris, M. A. et al. The Gene Ontology (GO) database and informatics resource. Nucleic acids research 32, D258-261, doi:10.1093/nar/gkh036 (2004). 